\title{
EuroMInd-C: a Disaggregate Monthly Indicator of Economic Activity for the Euro Area and member countries
}

\author{
Stefano Grassi ${ }^{1}$ \\ School of Economics, University of Kent and CREATES \\ Tommaso Proietti ${ }^{2}$ \\ Cecilia Frale ${ }^{3}$ \\ Università di Roma "Tor Vergata" and CREATES Italian Ministry of Education \\ Massimiliano Marcellino ${ }^{4}$ \\ Bocconi University, IGIER and CEPR \\ Gianluigi Mazzi ${ }^{5}$ \\ Eurostat
}

(C)2015. This manuscript version is made available under the CC-BY-NC-ND 4.0 license http:// creativecommons.org/licenses/by-nc-nd/4.0/

\footnotetext{
${ }^{1}$ School of Economics, University of Kent and CREATES. E-mail: S.Grassi@kent.ac.uk

${ }^{2}$ Corresponding author. Dipartimento di Economia e Finanza, Via Columbia 2, 00133 Roma, Italy. E-mail: tommaso.proietti@uniroma2.it.

${ }^{3}$ Ministry of Education, via XX Settembre 97, 00185 Roma, Italy. E-mail: cecilia.frale@ miur.it.

${ }^{4}$ Bocconi University, IGIER and CEPR. E-mail: massimiliano.marcellino@unibocconi.it

${ }^{5}$ Eurostat, Luxembourg. E-mail: Gianluigi.Mazzi@ec.europa.eu.
} 


\begin{abstract}
The paper deals with the estimation of monthly indicators of economic activity for the Euro area and its largest member countries that possess the following attributes: relevance, representativeness and timeliness. Relevance is determined by comparing our monthly indicators to the gross domestic product at chained volumes, as the most important measure of the level of economic activity. Representativeness is achieved by considering a very large number of (timely) time series of monthly indicators relating to the level of economic activity, providing a more or less complete coverage. The indicators are modelled using a large-scale parametric factor model. We discuss its specification and provide details of the statistical treatment. Computational efficiency is crucial for the estimation of large-scale parametric factor models of the dimension used in our application (considering about 170 series). To achieve it, we apply state-of-the-art state space methods that can handle temporal aggregation, and any pattern of missing values.
\end{abstract}

Keywords: Index of coincident indicators; Temporal disaggregation; Multivariate state space models; Dynamic factor models; Quarterly national accounts.

J.E.L. Classification: E32, E37, C53 


\section{Introduction}

Large-scale factor models aim to extract the main economic signals from a very large number of time series. The underlying idea is that the comovements among economic time series can be ascribed to a small number of common factors. Their two most prominent areas of application relate to the construction of synthetic indicators, such as coincident indicators of real economic activity $(?, ?, ?)$ and core inflation (?), and forecasting macroeconomic variables (?, ?); see ? for a survey.

Most of the factor-based studies use non-parametric techniques, which make estimation feasible even with a large dataset. Parametric large scale factor models have been recently entertained by ?, ?, and ?. Most of these studies rely on the Kalman filter and smoothing algorithms to enable them to handle mixed data frequencies and generic patterns of missing observations.

In this paper, we present a generalization of EuroMInd (see ?) known as EuroMInd-C, which allows for the simultaneous calculation of monthly indicators of economic activity for the Euro area and its largest member states. EuroMInd-C is based on a large-scale parametric factor model for handling very large sets of time series, with mixed frequencies, and subject to missing values, due chiefly to ragged-edged data structures, which depend in turn on the publication and dissemination schedules of those producing the data.

EuroMInd-C is constructed based on more than 100 monthly time series and 55 quarterly national accounts series. The latter concern the decomposition of the gross domestic product according to the output and expenditure approaches, for the Euro area as a whole and for its four largest countries (Germany, France, Italy and Spain). Indeed, a distinctive trait of our approach is the consideration of the quarterly national accounts estimates ${ }^{1}$ As a matter of fact, our dataset features a total of 11 GDP components, seven of which are drawn from the following decomposition of GDP from the output side:

\begin{tabular}{llr} 
Label & Value added by branch & \\
A-B & Agriculture, hunting, forestry and fishing & + \\
C-D-E & Industry, incl. energy & + \\
F & Construction & + \\
G-H-I & Trade, transport and communication services & + \\
J-K & Financial services and business activities & + \\
L-P & Other services & $=$ \\
\cline { 2 - 3 } & Total gross value added & + \\
TIS & Taxes less subsidies on products & $=$ \\
\cline { 2 - 3 } & GDP at market prices
\end{tabular}

The breakdown of total GDP from the expenditure side is as follows:

\footnotetext{
${ }^{1}$ Typically, the information set used for the estimation of factor models is strongly unbalanced towards the series collected from the supply side of the economy, namely establishments surveys (e.g., industrial production and turnover, retail sales, financial statistics), and from administrative records (e.g., building permits and car registrations). However, important information from other institutional units and economic agents, such as households, is missed, just because the underlying measurement process is more complex, with the consequence that the information becomes available with longer delays. Notable examples are the Labor Force Survey and the Consumer Expenditure Survey, which are carried out by the Euro area member states and represent essential sources of data on the labor market and consumption. On the other hand, this information is incorporated in the national accounts estimates of gross domestic product (GDP) and its components.
} 


\begin{tabular}{lll} 
Label & Component \\
FCE & Final consumption expenditure & + \\
GCF & Gross capital formation & + \\
EXP & Exports of goods and services & - \\
IMP & Imports of goods and services & $=$ \\
\cline { 2 - 2 } & GDP at market prices
\end{tabular}

With respect to methodology, we propose a large scale factor model such that the comovements among the series are synthesized by a set of 16 common factors, representing the Euro area common trend, four country-specific factors and 11 component-specific factors. The statistical treatment is based on likelihood inferences for a suitable state space model that is able to accommodate temporal aggregation and any pattern of missing data that arises, also taking into consideration the ragged-edge structure of the dataset and the release schedule of the economic indicators. Computationally efficient algorithms have been implemented to avoid the curse of dimensionality and to address specific issues related to the available data. For instance, for the extraction of the common factors, we adopt a reduction technique that reduces the dimension of the model to be equal to the number of common factors.

Our model is an extension of the single index model originally proposed by ?, and generalized by ?, jointly modelling quarterly GDP and monthly indicators, see also ? and ?. Our model also extends that of ?, who use a state space framework to link up a set of parametric mixed frequency factor models, one for each GDP demand and supply component of Euro area GDP, providing an indicator called EuroMInd. Unlike the original EuroMInd, the estimation of the monthly indicators of GDP and its components here is not carried out one component at a time, but simultaneously. In fact, the model includes all of the components of the breakdown of GDP, rather than performing a separate estimation for each different component (sector or expenditure type). Moreover, not only Euro area but country information is used, and country indicators of economic conditions are also produced. Unlike the model of ?, where a large scale factor model for the Euro area is estimated with an ex post identification of the factors, the factors in this model are identified a priori and ascribed to a particular effect (country-specific factors, factors pertaining to a specific GDP component).

The model is specified at the monthly frequency and for the logarithms of the original series. The presence of national account aggregates, which are observed only quarterly, imposes constraints in relation to temporal aggregation. Furthermore, due to the logarithmic specification, a nonlinear smoothing algorithm has to be applied for the disaggregation of the quarterly national account aggregates. The corresponding state space representation is modified according to the observational constraints so as to handle the temporal aggregation and the ragged-edged data structure and missing values at the beginning of the sample period.

Maximum likelihood estimation is carried out using the EM algorithm. As the number of time series being handled simultaneously is very large (around 170), we implement efficient methods of inference, focusing on the treatment of missing values and maximum likelihood estimation, as is documented in the following sections. Furthermore, the reduction technique proposed by ? and ? is applied to the temporal disaggregation case. This further extends the work of ? and ?.

The plan of the paper is as follows: Section 2 illustrates the specification of the dynamic factor model, and in particular the definition and identification of the common factors; and Section 3 describes the state space representation of the model. The statistical treatment of temporal aggregation is dealt with in Section 4, while Section 5 considers the missing values problem. Next, Section 6 discusses the estimation of common factors, and Section 7 the estimation of the other model parameters. The empirical application starts in Section 8, which provides a description of the dataset, with Section 9 then presenting the estimation results and the resulting EuroMInd-C estimates. Section 10 provides a comparative 
assessment of EuroMInd-C in terms of its predictive accuracy for quarterly GDP and its components, by means of a recursive forecasting experiment. Finally, Section 11 summarizes our main findings and concludes.

\section{Model specification}

This section outlines the basic structure of the dynamic factor model used for estimating EuroMInd-C. The specification can be considered as an extension of the single index model proposed by ?, introducing multiple indices relating to the countries and the sectors.

For illustrative purposes, we assume that a complete set of monthly time series is available. Let $c=$ $1, \ldots, C$ index a country, with $c=1$ indexing the Euro area, and let $s=1, \ldots, S$ denote a particular component (e.g., the construction sector, or an expenditure component such as exports); in our particular application, $C=5$ and $S=11$. Denoting the monthly series (usually transformed into logarithms) by $y_{c s, t}, t=0, \ldots, n$, for an indicator referring to country $c$ and component $s$, we write:

$$
y_{c s, t}=\theta_{c s, 0} \mu_{t}+\theta_{c s, 1} \mu_{c t}+\theta_{c s, 2} \mu_{s t}+\mu_{c s, t}^{*},
$$

where $\mu_{t}$ is the common Euro area factor, specified as an ARIMA $(1,1,0)$ process:

$$
\Delta \mu_{t}=\phi \Delta \mu_{t-1}+\eta_{t}, \quad \eta_{t} \sim \operatorname{NID}(0,1) .
$$

The factor $\mu_{c t}$ is specific to country $c$, whereas $\mu_{s t}$ is a factor that is specific to sector $s$. Both are specified as ARIMA $(1,1,0)$ processes:

$$
\begin{aligned}
\Delta \mu_{c t}=\phi_{c} \Delta \mu_{c, t-1}+\eta_{c t}, & \eta_{c t} \sim \operatorname{NID}(0,1), \\
\Delta \mu_{s t}=\phi_{s} \Delta \mu_{s, t-1}+\eta_{s t}, & \eta_{s t} \sim \operatorname{NID}(0,1) .
\end{aligned}
$$

Finally, the idiosyncratic component is formulated as follows:

$$
\Delta \mu_{c s, t}^{*}=\left(1-\psi_{c s}\right) \delta_{c s}+\psi_{c s} \Delta \mu_{c s, t-1}^{*}+\epsilon_{c s, t}, \quad \epsilon_{c s, t} \sim \operatorname{NID}\left(0, \sigma_{\epsilon, c s}^{2}\right) .
$$

The model assumes that each individual time series is made stationary by the transformation $\Delta y_{c s, t}=$ $y_{c s, t}-y_{c s, t-1}$ for $t=1, \ldots, n$. If $y_{c s, t}$ is a survey series, then the specification refers to the cumulated values of the series (which are by construction integrated of order 1). Note that the drift $\delta_{c s}$ is assigned to the idiosyncratic component, and is thus a series-specific feature.

For the Euro area as a whole $(c=1)$, the $j$ th series results from the aggregation of the series for all of the member countries. Thus, it will load on the global factor, the sector-specific factor, and the countryspecific factors of the individual member states under evaluation (the four largest economies). The specific factors of the countries not included in the model will contribute to the idiosyncratic component of the Euro area. However, the latter can be correlated with the idiosyncratic components of the countries included in the model. Thus, the equation for the Euro area series is specified as follows:

$$
y_{1 s, t}=\theta_{1 s, 0} \mu_{t}+\sum_{c=2}^{C} \theta_{1 c, 1} \mu_{c t}+\theta_{1 s, 2} \mu_{s t}+\mu_{1 s, t}^{*} .
$$

In general, to show the identification restrictions imposed on the loadings matrix, we report an example for five countries (EA, G, S, F and I, referring to the Euro area, Germany, Spain, France and Italy, respectively) and two sectors ( $a$ and $b$ ); note that our study includes a total of five countries and 11 
sectors). The value added series for the two sectors are listed first, and three monthly indicators are available: one refers to both sectors $\left(\operatorname{Ind} 1_{a, b}\right)$, while the other two refer to the first $\left(\operatorname{Ind} 2_{a}\right)$ and second $\left(\operatorname{Ind} 3_{b}\right)$ sectors, respectively.

$$
\left.\begin{array}{c}
E A_{a} \\
G_{a} \\
S_{a} \\
F_{a} \\
I_{a} \\
E A_{b} \\
G_{b} \\
S_{b} \\
F_{b} \\
I_{b} \\
E A-I n d 1_{a, b} \\
G-I n d 1_{a, b} \\
S-I n d 1_{a, b} \\
F-I n d 1_{a, b} \\
I-I n d 1_{a, b} \\
E A-I n d 2_{a} \\
G-I n d 2_{a} \\
S-I n d 2_{a} \\
F-I n d 2_{a} \\
I-I n d 2_{a} \\
E A-I n d 3_{b} \\
G-I n d 3_{b} \\
S-I n d 3_{b} \\
F-I n d 3_{b} \\
I-I n d 3_{b}
\end{array}\right)=\left(\begin{array}{lllllll}
x & x & x & x & x & x & 0 \\
x & x & 0 & 0 & 0 & x & 0 \\
x & 0 & x & 0 & 0 & x & 0 \\
x & 0 & 0 & x & 0 & x & 0 \\
x & 0 & 0 & 0 & x & x & 0 \\
x & x & x & x & x & 0 & x \\
x & x & 0 & 0 & 0 & 0 & x \\
x & 0 & x & 0 & 0 & 0 & x \\
x & 0 & 0 & x & 0 & 0 & x \\
x & 0 & 0 & 0 & x & 0 & x \\
x & x & x & x & x & x & x \\
x & x & 0 & 0 & 0 & x & x \\
x & 0 & x & 0 & 0 & x & x \\
x & 0 & 0 & x & 0 & x & x \\
x & 0 & 0 & 0 & x & x & x \\
x & x & x & x & x & x & 0 \\
x & x & 0 & 0 & 0 & x & 0 \\
x & 0 & x & 0 & 0 & x & 0 \\
x & 0 & 0 & x & 0 & x & 0 \\
x & 0 & 0 & 0 & x & x & 0 \\
x & x & x & x & x & 0 & x \\
x & x & 0 & 0 & 0 & 0 & x \\
x & 0 & x & 0 & 0 & 0 & x \\
x & 0 & 0 & x & 0 & 0 & x \\
x & 0 & 0 & 0 & x & 0 & x
\end{array}\right)\left(\begin{array}{c}
\mu_{t} \\
\mu_{G, t} \\
\mu_{S, t} \\
\mu_{F, t} \\
\mu_{I, t} \\
\mu_{a, t} \\
\mu_{b, t}
\end{array}\right)+\boldsymbol{\mu}_{t}^{*}
$$

Here, $\boldsymbol{\mu}_{t}^{*}$ is a vector of idiosyncratic components. The first factor, $\mu_{t}$, is interpreted as the Euro area common factor. The factors $\mu_{G, t}, \mu_{S, t}, \mu_{F, t}, \mu_{I, t}$ are country-specific, and, finally, the factors $\mu_{a, t}$ and $\mu_{b, t}$ are sector-specific. All of the time series load on the Euro area factor and on the factor of the component to which they refer. Hence, for instance, the German value added for sector $b, G_{b}$, as well as $G-\operatorname{Ind} 3_{b}$, load on the common Euro area factor, the German factor and the industry $b$ factor. The German and French sector $a$ indicators are related only via the sector-specific factor $\mu_{a, t}$ and the common Euro area factor $\mu_{t}$. All of the series are related via the latter.

The Euro area is treated as a separate country; its treatment is similar to that assigned to the U.S. by ? and ?. It would also be possible to model the rest of the Euro area as an additional entity and obtain the Euro area aggregates by summing the five series pertaining to the four countries and the residual unit. While this is feasible technically, the main issue is to find a representative set of indicators for the remaining Euro area countries.

Finally, the model is specified at the monthly frequency. However, the national accounts series, and the quarterly series more generally, are not observed at that frequency. Sections 3-7 deal with the more technical aspects of the model's specification and estimation, and can be skipped by readers who are not interested in these details. 


\section{The dynamic factor model}

First of all, we present the specification of the model as a dynamic factor model in the first log-differences of the series, assuming that a complete data set of monthly observations is available at time $t=1, \ldots, n$.

We start by denoting the stack of the $N$ individual monthly time series $Y_{c s, t}, c=1, \ldots, C, s=1, \ldots, S$, in their original scale of measurement by $\boldsymbol{Y}_{t}, t=0, \ldots, n$. Further, let us denote a transformation of $\boldsymbol{Y}_{t}$ by $\boldsymbol{y}_{t}$. We assume throughout that $y_{c s, t}=\ln Y_{c s, t}$ (log transformation) for all of the series measured on a ratio scale, such as the components of GDP, the index of industrial production, etc. For the survey variables (which can take on negative values), we always set $y_{c s, t}=Y_{c s, t}$ (no transformation).

For ease of notation, henceforth we will adopt a single index $i$ to refer to the $i$ th element of the $N \times 1$ vector $\boldsymbol{Y}_{t}$. Stacking the individual equations for $\Delta y_{i t}$, the model is formulated as follows:

$$
\begin{aligned}
& \Delta \boldsymbol{y}_{t}=\boldsymbol{\delta}+\boldsymbol{\Theta} \boldsymbol{f}_{t}+\boldsymbol{u}_{t}, \quad t=1, \ldots, n, \\
& \boldsymbol{f}_{t}=\boldsymbol{\Phi} \boldsymbol{f}_{t-1}+\boldsymbol{\eta}_{t}, \quad \boldsymbol{\eta}_{t} \sim \operatorname{NID}\left(\mathbf{0}, \boldsymbol{\Sigma}_{\eta}\right), \\
& \boldsymbol{u}_{t}=\boldsymbol{\Psi} \boldsymbol{u}_{t-1}+\boldsymbol{\epsilon}_{t}, \quad \boldsymbol{\epsilon}_{t} \sim \operatorname{NID}\left(\mathbf{0}, \boldsymbol{\Sigma}_{\epsilon}\right),
\end{aligned}
$$

where $\delta=\left\{\delta_{c s}\right\}$ contains the drifts in Eq. (3), $\boldsymbol{f}_{t}$ is a $K \times 1$ vector of stationary common factors, with zero mean, stacking the first differences $\left[\Delta \mu_{t}, \Delta \mu_{c t}, c=2, \ldots, C, \Delta \mu_{s t}, s=1, \ldots, S\right]$. The total number of common factors is denoted by $K=C+S$, and $\boldsymbol{\Theta}=\left[\boldsymbol{\theta}_{1}, \ldots, \boldsymbol{\theta}_{i}, \ldots, \boldsymbol{\theta}_{N}\right]^{\prime}$ is the $N \times K$ matrix of factor loadings,

$$
\mathbf{\Phi}=\operatorname{diag}\left\{\phi_{1}, \ldots, \phi_{k}, \ldots, \phi_{K}\right\},
$$

$\boldsymbol{\Sigma}_{\eta}=\boldsymbol{I}_{K}$, where $\boldsymbol{I}_{K}$ is the identity matrix of order $K$. Hence, $f_{k t}=\phi_{k} f_{k, t-1}+\eta_{k t}, \eta_{k t} \sim \operatorname{NID}(0,1)$. Finally, $\boldsymbol{u}_{t}$ is the $N \times 1$ vector of stationary idiosyncratic components, which arises from the decomposition

$$
\Delta \boldsymbol{\mu}_{t}^{*}=\boldsymbol{\delta}+\boldsymbol{u}_{t} .
$$

Therefore, the components of the vector $\boldsymbol{u}_{t}$ follow scalar independent AR(1) processes, $u_{i t}=\psi_{i} u_{i, t-1}+$ $\epsilon_{i t}, \epsilon_{i t} \sim \mathrm{NID}\left(0, \sigma_{i}^{2}\right)$, according to Eq. 33, so that

$$
\boldsymbol{\Psi}=\operatorname{diag}\left\{\psi_{1}, \ldots, \psi_{i}, \ldots, \psi_{N}\right\}, \quad \boldsymbol{\Sigma}_{\epsilon}=\operatorname{diag}\left\{\sigma_{1}^{2}, \ldots, \sigma_{i}^{2}, \ldots, \sigma_{N}^{2}\right\} .
$$

For later use, we also define the covariance matrix of the factors, $\Sigma_{f}$, which is given by $\boldsymbol{\Sigma}_{f}=\operatorname{diag}\{(1-$ $\left.\left.\phi_{k}^{2}\right)^{-1}\right\}, \quad k=1, \ldots, K$.

The model in Eq. (6) is identified by imposing exclusion restrictions on the matrix $\Theta$ that actually overidentify the model. The number of common factors is fixed at $K=16$, since we postulate the presence of a common global factor, as well as country- and sector-specific factors. The assumption that the common factors and the idiosyncratic components are uncorrelated, $\mathrm{E}\left(\boldsymbol{\epsilon}_{t} \boldsymbol{\eta}_{t}^{\prime}\right)=\mathbf{0}$, completes the specification.

Let us define the parameter vector $\boldsymbol{\Xi}=\left[\boldsymbol{\vartheta}^{\prime}, \boldsymbol{\delta}^{\prime}, \boldsymbol{\phi}^{\prime}, \boldsymbol{\psi}^{\prime}, \boldsymbol{\varsigma}_{\epsilon}^{\prime}\right]^{\prime}$, where $\boldsymbol{\vartheta}$ stacks the nonzero elements of the loadings matrix, $\boldsymbol{\phi}=\left[\phi_{1}, \ldots, \phi_{k}\right]^{\prime}, \boldsymbol{\Phi}=\operatorname{diag}(\boldsymbol{\phi}), \boldsymbol{\psi}=\left[\psi_{1}, \ldots, \psi_{N}\right]^{\prime}, \boldsymbol{\Psi}=\operatorname{diag}(\boldsymbol{\psi})$, and $\boldsymbol{\varsigma}_{\epsilon}=$ $\left[\sigma_{1}^{2}, \ldots, \sigma_{N}^{2}\right]^{\prime}, \boldsymbol{\Sigma}_{\epsilon}=\operatorname{diag}\left(\boldsymbol{\varsigma}_{\epsilon}\right)$. Using the above restrictions and $\sigma_{i}^{2}>0, \forall i$, the vector $\boldsymbol{\Xi}$ is globally identified.

If $\Delta \boldsymbol{y}_{t}$ were observed fully, inference concerning the model in Eq. (6) would be straightforward, though computationally demanding. For small values of $N$ (less than 10, say), the parameters can be estimated by maximum likelihood, where the likelihood is evaluated by the Kalman filter (KF) using a numerical quasi-Newton method. For large values of $N$, the high dimensionality of $\boldsymbol{\Xi}$ prevents the maximization of the likelihood via gradient-based methods. A computationally viable alternative is to use the expectationmaximization (EM) algorithm of ?; see ? and ?. 
Another issue is that, according to Eq. (6), $\boldsymbol{u}_{t}$ has a first order Markovian representation. If the model is written in state space form, the state vector should feature $N$ elements, to account for the idiosyncratic factors. Filtering and smoothing then becomes infeasible if $N$ has the dimension considered in this paper.

A transformation of the model that will be useful in what follows is the quasi-difference form:

$$
\Delta \boldsymbol{y}_{t}=\boldsymbol{\Psi} \Delta \boldsymbol{y}_{t-1}+\boldsymbol{\delta}-\boldsymbol{\Psi} \boldsymbol{\delta}+\boldsymbol{\Theta} \boldsymbol{f}_{t}-\boldsymbol{\Psi} \boldsymbol{\Theta} \boldsymbol{f}_{t-1}+\boldsymbol{\epsilon}_{t} .
$$

The main virtue of this representation is that the idiosyncratic VAR(1) process $\boldsymbol{u}_{t}$ has been removed, and only the idiosyncratic disturbances $\epsilon_{t}$ are present, though they are serially uncorrelated. The dimensionality of the unobserved components with a dynamic structure depends solely on the number of common factors, and thus, is much smaller than $N$. The inclusion of $\boldsymbol{u}_{t}$ in the state vector is necessary only for setting up the initial conditions. The reduction is achieved at the cost of conditioning upon $\Delta \boldsymbol{y}_{t-1}$; this will have certain consequences for the way in which we handle missing values, as we will see later.

New, computationally efficient methods of inference need to be applied for estimating the factors and the parameters. First and foremost, the specification of the model needs to take into consideration temporal aggregation, the presence of missing values, and the ragged edge structure of the data. The final state space representation will now be obtained in two stages:

- The original state space form that is suitable for Eq. (7) is modified to take into consideration temporal aggregation: the observational constraints are enforced sequentially by solving a nonlinear smoothing problem using a linearised Gaussian model, in Section 4.

- Missing values are dealt with using a time varying state space form, in Section 5.

\section{Temporal aggregation}

Our data set contains $N_{1}=55$ quarterly national accounts (NA) aggregates that are subject to temporal aggregation: the 11 GDP components for the five countries. These series represent a set of constraints for the monthly estimates. The remaining $N_{2}=N-N_{1}$ time series are a set of monthly coincident indicators referring to either the Euro area or the individual countries (e.g., index of industrial production, turnover, and so forth). They may be subject to isolated missing values and to a ragged edge structure, depending on their timeliness and publishing schedules. Accordingly, we partition the vector $\boldsymbol{Y}_{t}$ in two blocks, $\boldsymbol{Y}_{t}=\left[\boldsymbol{Y}_{1 t}^{\prime}, \boldsymbol{Y}_{2 t}^{\prime}\right]^{\prime}$; and $\boldsymbol{y}_{t}$ and $\Delta \boldsymbol{y}_{t}$ will also be partitioned in two blocks correspondingly, where the first block refers to the quarterly national accounts series and the second to the monthly coincident indicators.

The monthly series $\boldsymbol{Y}_{1 t}$, and thus $\boldsymbol{y}_{i t}=\ln \boldsymbol{Y}_{1 t}, t=0,1, \ldots, n$, and $\Delta \boldsymbol{y}_{i t}$, for $i=1, \ldots, N_{1}$, are not observed for the first block of $N_{1}$ time series belonging to the national accounts. Instead, only the quarterly totals are observed, which are supposed to constrain the monthly estimates. This observational constraint can be incorporated in the specification of the model, and we will derive the relevant state space representation of the model later. The latter has two fundamental uses: first, for a given parameter configuration, it provides the optimal (posterior mode) estimates of the disaggregate monthly time series, the factors and the idiosyncratic components. Second, it is instrumental in performing the expectation step of the EM algorithm for the maximum likelihood estimation of the parameters. These evaluations are done via the nonlinear smoothing algorithm that is discussed in Section 4.1. For $Y_{i t}, i=1, \ldots, N_{1}$, subject to temporal aggregation, we observe the quarterly totals:

$$
Y_{i \tau}=\sum_{j=1}^{3} Y_{i, 3 \tau-j}, \quad \tau=1,2, \ldots,[(n+1) / 3],
$$


Table 1: Nonlinear temporal aggregation.

\begin{tabular}{ccclc}
\hline$t$ & Unobserved $Y_{i t}$ & Logarithms & Cumulator & Availability of $Y_{i t}^{c}$ \\
\hline 0 & $Y_{i 0}$ & $y_{i 0}$ & $Y_{i 0}^{c}=Y_{i 0}=\exp \left(y_{i 0}\right)$ & missing \\
1 & $Y_{i 1}$ & $y_{i 1}$ & $Y_{i 1}^{c}=Y_{i 0}+Y_{i 1}=\exp \left(y_{i 0}\right)+\exp \left(y_{i 1}\right)$ & missing \\
2 & $Y_{i 2}$ & $y_{i 2}$ & $Y_{i 2}^{c}=Y_{i 0}+Y_{i 1}+Y_{i 2}=\exp \left(y_{i 0}\right)+\exp \left(y_{i 1}\right)+\exp \left(y_{i 2}\right)$ & observed \\
3 & $Y_{i 3}$ & $y_{i 3}$ & $Y_{i 3}^{c}=Y_{i 3}=\exp \left(y_{i 3}\right)$ & missing \\
4 & $Y_{i 4}$ & $y_{i 4}$ & $Y_{i 4}^{c}=Y_{i 3}+Y_{i 4}=\exp \left(y_{i 3}\right)+\exp \left(y_{i 4}\right)$ & missing \\
5 & $Y_{i 5}$ & $y_{i 5}$ & $Y_{i 5}^{c}=Y_{i 3}+Y_{i 4}+Y_{i 5}=\exp \left(y_{i 3}\right)+\exp \left(y_{i 4}\right)+\exp \left(y_{i 5}\right)$ & observed \\
6 & $Y_{i 6}$ & $y_{i 6}$ & $Y_{i 6}^{c}=Y_{i 6}=\exp \left(y_{i 6}\right)$ & missing \\
$\vdots$ & $\vdots$ & $\vdots$ & $\vdots$ & \\
\hline
\end{tabular}

where $[\cdot]$ is the integer part of the argument.

For the statistical treatment, it is useful to convert temporal aggregation into a systematic sampling problem; this can be done by constructing a cumulator variable, generated by the recursive formula (see ?, and ?):

$$
\begin{aligned}
Y_{i t}^{c} & =\rho_{t} Y_{i, t-1}^{c}+Y_{i t}, \quad t=0, \ldots, n \\
& =\rho_{t} Y_{i, t-1}^{c}+\exp \left(y_{i t}\right),
\end{aligned}
$$

where $\rho_{t}$ is the cumulator coefficient, which is equal to zero for $t$ corresponding to the first month in the quarter and 1 otherwise, i.e.,

$$
\rho_{t}= \begin{cases}0, & t=3(\tau-1), \quad \tau=1, \ldots,[(n+1) / 3] \\ 1, & \text { otherwise }\end{cases}
$$

Only a systematic sample of the cumulator variable $Y_{i t}^{c}$ is available; in particular, if the sample period starts with the first month of the quarter at $t=0$, the observed end-of-quarter values occur at times $t=3 \tau-1, \tau=1,2, \ldots,[(n+1) / 3]$. In our particular case, $Y_{i 0}^{c}=\exp \left(y_{i 0}\right), Y_{i 1}^{c}=\exp \left(y_{i 0}\right)+\exp \left(y_{i 1}\right)$, $Y_{i 2}^{c}=\exp \left(y_{i 0}\right)+\exp \left(y_{i 1}\right)+\exp \left(y_{i 2}\right), Y_{i 3}^{c}=\exp \left(y_{i 3}\right), Y_{i 4}^{c}=\exp \left(y_{i 3}\right)+\exp \left(y_{i 4}\right), Y_{i 5}^{c}=\exp \left(y_{i 3}\right)+$ $\exp \left(y_{i 4}\right)+\exp \left(y_{i 5}\right), \ldots$ As is illustrated in Table 1, only the values $Y_{i 2}^{c}, Y_{i 5}^{c}, \ldots$ are observed, with the intermediate ones being missing. Temporal aggregation yields a nonlinear observational constraint, since the quarterly totals are a nonlinear function of the underlying monthly values $y_{i t}$.

\subsection{An approximating linear Gaussian model}

The estimation of the common factors $\boldsymbol{f}_{t}$, and the missing values $\boldsymbol{y}_{i t}, i=1, \ldots, N_{1}$, conditional on $\boldsymbol{\Xi}$ and the available information, which consists of $Y_{i t}^{c}, i=1, \ldots, N_{1}, t=3 \tau-1, \tau=1,2, \ldots,[(n+1) / 3]$, for the quarterly time series, and $y_{i t}$ for $i=N_{1}+1, \ldots, N$, is a nonlinear smoothing problem that can be solved by iterating the Kalman filter and smoother (KFS) adapted to a sequentially linearized state space model, see ?.

Let us partition the vectors $\boldsymbol{Y}_{t}=\left[\boldsymbol{Y}_{1 t}^{\prime}, \boldsymbol{Y}_{2 t}^{\prime}\right]^{\prime}, \boldsymbol{y}_{t}=\left[\boldsymbol{y}_{1 t}^{\prime}, \boldsymbol{y}_{2 t}^{\prime}\right]^{\prime}$, such that $Y_{i t}=\exp \left(y_{i t}\right)$ for all of the series measured on a ratio scale (all of the series except the business survey variables), $\Delta \boldsymbol{y}_{t}=$ $\left[\Delta \boldsymbol{y}_{1 t}^{\prime}, \Delta \boldsymbol{y}_{2 t}^{\prime}\right]^{\prime}, \boldsymbol{\delta}=\left[\boldsymbol{\delta}_{1}, \boldsymbol{\delta}_{2}\right]^{\prime}, \boldsymbol{u}_{t}=\left[\boldsymbol{u}_{1 t}, \boldsymbol{u}_{2 t}\right]^{\prime}$ and the matrices $\boldsymbol{\Theta}=\left[\boldsymbol{\Theta}_{1}^{\prime}, \boldsymbol{\Theta}_{2}^{\prime}\right]^{\prime}, \boldsymbol{\Psi}=\operatorname{diag}\left(\boldsymbol{\Psi}_{1}, \boldsymbol{\Psi}_{2}\right)$, $\boldsymbol{\Sigma}_{\epsilon}=\operatorname{diag}\left(\boldsymbol{\Sigma}_{\epsilon 1}, \boldsymbol{\Sigma}_{\epsilon 2}\right)$, where the subscript 1 indexes the national accounts series, and the dimensions of the blocks are respectively $N_{1}$ and $N_{2}$. Also, define $\boldsymbol{u}=\left[\boldsymbol{u}_{1}^{\prime}, \ldots, \boldsymbol{u}_{n}^{\prime}\right]^{\prime}$, and $\boldsymbol{f}=\left[\boldsymbol{f}_{1}^{\prime}, \ldots, \boldsymbol{f}_{n}^{\prime}\right]^{\prime}$. Although $\Delta \boldsymbol{y}_{2 t}$ is available, $\Delta \boldsymbol{y}_{1 t}, t=1, \ldots, n$, is not, but we observe a systematic sample of

$$
\begin{aligned}
\boldsymbol{Y}_{1 t}^{c} & =\rho_{t} \boldsymbol{Y}_{1, t-1}^{c}+\boldsymbol{Y}_{1 t}, \\
& =\rho_{t} \boldsymbol{Y}_{1, t-1}^{c}+\exp \left(\boldsymbol{y}_{1 t}\right) .
\end{aligned}
$$


To obtain the values $\boldsymbol{f}$ and $\boldsymbol{u}$ that maximize the posterior density $g(\boldsymbol{f}, \boldsymbol{u} \mid \boldsymbol{x} ; \boldsymbol{\Xi})$, given the available data,we linearize $\boldsymbol{Y}_{1 t}^{c}=\rho_{t} \boldsymbol{Y}_{1, t-1}^{c}+\exp \left(\boldsymbol{y}_{1 t}\right)$ around a trial value $\boldsymbol{y}_{1 t}^{*}$, using a first order Taylor series expansion. This yields a linear state space model, and the corresponding KFS provides a new trial value for the disaggregate series. This sequence of linearisations is iterated until convergence, with the end result being a set of disaggregate monthly estimates $\boldsymbol{Y}_{1}$ and factor scores $\boldsymbol{f}$ which incorporate the temporal aggregation constraints. As a by-product, disaggregate (monthly) estimates of the missing values $\boldsymbol{y}_{1 t}$, and thus $\boldsymbol{Y}_{i t}=\exp \left(\boldsymbol{y}_{1 t}\right)$, will be made available. Using the trial value $\boldsymbol{y}_{1 t}^{*}=\log \left(\boldsymbol{Y}_{1 t}^{*}\right)$, the linearisation operates as follows:

$$
\boldsymbol{Y}_{1 t}^{c}=\rho_{t} \boldsymbol{Y}_{1, t-1}^{c}+\exp \left(\boldsymbol{y}_{1 t}^{*}\right)+\boldsymbol{U}_{1 t}^{*}\left(\boldsymbol{y}_{1 t}-\boldsymbol{y}_{1 t}^{*}\right),
$$

where the $N_{1} \times N_{1}$ matrix $\boldsymbol{U}_{1 t}^{*}$ is a diagonal matrix with the derivatives of the exponential transformation on the main diagonal, $\boldsymbol{U}_{1 t}^{*}=\operatorname{diag}\left(\exp \left(\boldsymbol{y}_{1 t}^{*}\right)\right)$. Writing

$$
\begin{aligned}
\boldsymbol{y}_{1 t} & =\boldsymbol{y}_{1, t-1}+\Delta \boldsymbol{y}_{1 t} \\
& =\boldsymbol{y}_{1, t-2}+\Delta \boldsymbol{y}_{1, t-1}+\Delta \boldsymbol{y}_{1 t},
\end{aligned}
$$

and replacing $\Delta \boldsymbol{y}_{1 t}=\boldsymbol{\Psi}_{1} \Delta \boldsymbol{y}_{1 t-1}+\left(\boldsymbol{I}-\mathbf{\Psi}_{1}\right) \boldsymbol{\delta}_{1}+\boldsymbol{\Theta}_{1} \boldsymbol{f}_{t}-\boldsymbol{\Psi}_{1} \boldsymbol{\Theta}_{1} \boldsymbol{f}_{t-1}+\boldsymbol{\epsilon}_{1 t}$, yields

$$
\boldsymbol{Y}_{1 t}^{c}=\rho_{t} \boldsymbol{Y}_{1 t-1}^{c}+\tilde{\boldsymbol{d}}_{t}^{*}+\boldsymbol{U}_{1 t}^{*}\left[\boldsymbol{y}_{1 t-2}+\left(\boldsymbol{I}+\boldsymbol{\Psi}_{1}\right) \Delta \boldsymbol{y}_{1 t-1}+\left(\boldsymbol{\Theta}_{1} \boldsymbol{\Phi}-\boldsymbol{\Psi}_{1} \boldsymbol{\Theta}_{1}\right) \boldsymbol{f}_{t-1}+\boldsymbol{\epsilon}_{1 t}+\boldsymbol{\Theta}_{1} \boldsymbol{\eta}_{t}\right],
$$

where $\tilde{\boldsymbol{d}}_{t}^{*}=\exp \left(\boldsymbol{y}_{1 t}^{*}\right)-\boldsymbol{U}_{1 t}^{*} \boldsymbol{y}_{1 t}+\boldsymbol{U}_{1 t}^{*}\left(\boldsymbol{I}-\boldsymbol{\Psi}_{N_{1}}\right) \boldsymbol{\delta}_{1}$. Eq. 10 expresses $\boldsymbol{Y}_{1 t}^{c}$ as a time-varying linear combination of $\boldsymbol{Y}_{1, t-1}^{c}, \boldsymbol{y}_{1, t-2}, \Delta \boldsymbol{y}_{1, t-1}$ and $\boldsymbol{f}_{t-1}$, which will constitute the elements of the state vector at time $t-1$, denoted by $\boldsymbol{\alpha}_{t-1}$, and of the disturbances of the factor model.

\subsection{A convenient state space formulation under temporal aggregation constraints}

The state space representation is formulated conveniently for the vector $\boldsymbol{y}_{t}^{\dagger}$, where, for $t \geq 1$, and if no element of $\boldsymbol{y}_{2 t}$ is missing,

$$
\boldsymbol{y}_{t}^{\dagger}=\left[\begin{array}{c}
\boldsymbol{Y}_{1 t}^{c} \\
\Delta \boldsymbol{y}_{2 t}
\end{array}\right], t=1,2, \ldots, n
$$

whereas for $t=0, \boldsymbol{y}_{0}^{\dagger}=\boldsymbol{Y}_{10}^{c}$. The length of the observation vector varies with time and will be denoted by $N_{t}$. When one or more elements of $\boldsymbol{y}_{2 t}$ are missing, the measurement equation will be modified suitably, as it will be discussed later.

When $\Delta \boldsymbol{y}_{2 t}$ is observed fully, the state vector for $t \geq 1$ is defined as follows:

$$
\boldsymbol{\alpha}_{t}=\left[\begin{array}{c}
\boldsymbol{Y}_{1, t}^{c} \\
\boldsymbol{y}_{1, t-1} \\
\Delta \boldsymbol{y}_{1 t} \\
\Delta \boldsymbol{y}_{1, t-1} \\
\boldsymbol{f}_{t} \\
\boldsymbol{f}_{t-1}
\end{array}\right] .
$$

The length of the state vector will also vary according to $t$ and to the presence of missing values.

The measurement equation is formulated as follows:

$$
\left[\begin{array}{c}
\boldsymbol{Y}_{1 t}^{c} \\
\Delta \boldsymbol{y}_{2 t}
\end{array}\right]=\left[\begin{array}{cccccc}
\boldsymbol{I}_{N_{1}} & \mathbf{0} & \mathbf{0} & \mathbf{0} & \mathbf{0} & \mathbf{0} \\
\mathbf{0} & \mathbf{0} & \mathbf{0} & \mathbf{0} & \boldsymbol{\Theta}_{2} & -\boldsymbol{\Psi}_{2} \boldsymbol{\Theta}_{2}
\end{array}\right] \boldsymbol{\alpha}_{t}+\left[\begin{array}{c}
\mathbf{0} \\
\left(\boldsymbol{I}-\boldsymbol{\Psi}_{2}\right) \boldsymbol{\delta}_{2}+\boldsymbol{\Psi}_{2} \Delta \boldsymbol{y}_{2 t-1}
\end{array}\right]+\left[\begin{array}{ccc}
\mathbf{0} & \mathbf{0} & \mathbf{0} \\
\mathbf{0} & \mathbf{0} & \boldsymbol{I}
\end{array}\right]\left[\begin{array}{c}
\boldsymbol{\eta}_{t} \\
\boldsymbol{\epsilon}_{1 t} \\
\boldsymbol{\epsilon}_{2 t}
\end{array}\right]
$$


The transition equation is:

$$
\begin{aligned}
{\left[\begin{array}{c}
\boldsymbol{Y}_{1 t}^{c} \\
\boldsymbol{y}_{1 t-1} \\
\Delta \boldsymbol{y}_{1 t} \\
\Delta \boldsymbol{y}_{1 t-1} \\
\boldsymbol{f}_{t} \\
\boldsymbol{f}_{t-1}
\end{array}\right]=} & {\left[\begin{array}{cccccc}
\rho_{t} \boldsymbol{I}_{N 1} & \boldsymbol{U}_{1 t}^{*} & \boldsymbol{U}_{1 t}^{*}\left(\boldsymbol{I}+\boldsymbol{\Psi}_{1}\right) & \mathbf{0} & \boldsymbol{U}_{1 t}^{*}\left[\boldsymbol{\Theta}_{1} \boldsymbol{\Phi}-\boldsymbol{\Psi}_{1} \boldsymbol{\Theta}_{1}\right] & \mathbf{0} \\
\mathbf{0} & \boldsymbol{I}_{N 1} & \boldsymbol{I}_{N 1} & \mathbf{0} & \mathbf{0} & \mathbf{0} \\
\mathbf{0} & \mathbf{0} & \boldsymbol{\Psi}_{N_{1}} & \mathbf{0} & {\left[\boldsymbol{\Theta}_{1} \boldsymbol{\Phi}-\boldsymbol{\Psi}_{1} \boldsymbol{\Theta}_{1}\right]} & \mathbf{0} \\
\mathbf{0} & \mathbf{0} & \boldsymbol{I} & \mathbf{0} & \mathbf{0} & \mathbf{0} \\
\mathbf{0} & \mathbf{0} & \mathbf{0} & \mathbf{0} & \mathbf{\Phi} & \mathbf{0} \\
\mathbf{0} & \mathbf{0} & \mathbf{0} & \mathbf{0} & \boldsymbol{I} & \mathbf{0}
\end{array}\right]\left[\begin{array}{c}
\boldsymbol{Y}_{1 t-1}^{c} \\
\boldsymbol{y}_{1 t-2} \\
\Delta \boldsymbol{y}_{1 t-1} \\
\Delta \boldsymbol{y}_{1 t-2} \\
\boldsymbol{f}_{t-1} \\
\boldsymbol{f}_{t-2}
\end{array}\right] } \\
+ & {\left[\begin{array}{c}
\boldsymbol{Y}_{1 t}^{*}-\boldsymbol{U}_{1 t}^{*} \boldsymbol{y}_{1 t}^{*}+\boldsymbol{U}_{1 t}^{*}\left(\boldsymbol{I}-\boldsymbol{\Psi}_{N_{1}}\right) \boldsymbol{\delta}_{1} \\
\mathbf{0} \\
\left(\boldsymbol{I}-\boldsymbol{\Psi}_{N_{1}}\right) \boldsymbol{\delta}_{1} \\
\mathbf{0} \\
\mathbf{0}
\end{array}\right]+\left[\begin{array}{ccc}
\boldsymbol{U}_{1 t} \boldsymbol{\Theta}_{1} & \boldsymbol{U}_{1 t}^{*} & \mathbf{0} \\
\mathbf{0} & \mathbf{0} & \mathbf{0} \\
\boldsymbol{\Theta}_{1} & \boldsymbol{I}_{N_{1}} & \mathbf{0} \\
\mathbf{0} & \mathbf{0} & \mathbf{0} \\
\boldsymbol{I}_{k} & \mathbf{0} & \mathbf{0} \\
\mathbf{0} & \mathbf{0} & \mathbf{0}
\end{array}\right]\left[\begin{array}{c}
\boldsymbol{\eta}_{t} \\
\boldsymbol{\epsilon}_{1 t} \\
\boldsymbol{\epsilon}_{2 t}
\end{array}\right] . }
\end{aligned}
$$

The treatment of initial conditions is rather technical, and hence, is relegated to Appendix C.

\section{The treatment of missing values}

Expressing the model in quasi differences as in Eq. (7) offers the advantage of dropping the $\mathrm{N}$ dimensional vector $\boldsymbol{u}_{t}$ from the unobserved states. However, it introduces a new difficulty when the monthly indicator series $\boldsymbol{y}_{2 t}$, or any element thereof, is missing at time $t$. As a matter of fact, a missing value at time $t$ implies not only that the pair $\left(\Delta \boldsymbol{y}_{t}, \Delta \boldsymbol{y}_{t+1}\right)$ are subject to missing values, but also that the conditioning elements on the right hand side of the Eq. (7) are missing.

To overcome this difficulty, the state space formulation needs to be adapted by augmenting the state vector with the minimal set of components needed to preserve the dynamic structure of the system and its Markovian structure. Our treatment is based on an adaptation of the approach recently proposed by ?. The main advantages of this formulation lies in its treatment of arbitrary patterns of missing values.

We need to discriminate between the following situations: in the case when both $\boldsymbol{y}_{t}$ and $\boldsymbol{y}_{t-1}$ are observed, the state space formulation considered in the previous section applies. The state space form has to be modified in the case of missing values. The three main cases of interest are:

1. Case 1: $\boldsymbol{y}_{t}$ (or any component series thereof) is missing at time $t$, but is observed at time $t-1$.

2. Case 2: $\boldsymbol{y}_{t}$ (or any component series thereof) is missing at both time $t$ and time $t-1$.

3. Case 3: $\boldsymbol{y}_{t}$ (or any component series thereof) is observed at time $t$, but is missing at time $t-1$.

Details of the different treatments of the three cases are provided in Appendix C.

\section{A reduction technique for the estimation of the common factors}

The smoothed estimates of the factors conditional on the parameter vector $\boldsymbol{\Xi}$ can be obtained efficiently via an adaptation of a reduction technique, entailing a linear transformation of the model that operates a projection into the space of the factors. This will be useful for the E-step of the EM algorithm.

The computational effort involved in the KFS depends on the dimensions of both the state vector and the observation vectors. In most practical applications of the dynamic factor model, the dimension of 
$\boldsymbol{y}_{t}$ is significantly larger than that of $\boldsymbol{\alpha}_{t}$. ? demonstrated that in such circumstances, the computational effort of KFS can be improved significantly by a transformation of the model. Recently, ? used this computational device for the estimation of the common factors for large scale models.

We illustrate the technique with respect to a generic state space form, represented as follows:

$$
\begin{aligned}
& \boldsymbol{y}_{t}=\boldsymbol{c}_{t}+\boldsymbol{Z}_{t} \boldsymbol{\alpha}_{t}+\boldsymbol{G}_{t} \boldsymbol{\omega}_{t}, \quad \boldsymbol{\omega}_{t} \sim \mathrm{N}\left(\mathbf{0}, \boldsymbol{\Sigma}_{\omega}\right), \\
& \boldsymbol{\alpha}_{t+1}=\boldsymbol{T}_{t} \boldsymbol{\alpha}_{t}+\boldsymbol{d}_{t}+\boldsymbol{H}_{t} \boldsymbol{\omega}_{t},
\end{aligned}
$$

where for a $p$-dimensional state vector, $\boldsymbol{c}_{t}$ and $\boldsymbol{d}_{t}$ are suitable vectors of sizes $N \times 1$ and $p \times 1$ respectively; and $\boldsymbol{Z}_{t}, \boldsymbol{G}_{t}, \boldsymbol{\Sigma}_{\omega}, \boldsymbol{T}_{t}$ and $\boldsymbol{H}_{t}$ are suitable system matrices that are possibly time-varying.

The reduction technique is based on a linear transformation of the original model which projects the observations onto the space spanned by the states $\boldsymbol{\alpha}_{t}$ and its orthogonal complement. The information that is relevant for extracting the factors lies in the former, which is typically low-dimensional.

Define the transformation matrix

$$
\boldsymbol{A}_{t}=\left(\begin{array}{c}
\boldsymbol{A}_{t}^{L} \\
\boldsymbol{A}_{t}^{H}
\end{array}\right) \quad \boldsymbol{A}_{t}^{L}=\boldsymbol{C}_{t}^{-1} \boldsymbol{Z}_{t}^{\prime} \widetilde{\Sigma}_{t}^{-1}
$$

where $\widetilde{\boldsymbol{\Sigma}}_{t}=\boldsymbol{G}_{t} \boldsymbol{\Sigma}_{\omega} \boldsymbol{G}_{t}^{\prime}, \boldsymbol{C}_{t}$ is an invertible matrix, $\boldsymbol{A}_{t}^{H}$ is chosen such that matrix $\boldsymbol{A}_{t}$ is full rank, and $\boldsymbol{A}_{t}^{L} \widetilde{\boldsymbol{\Sigma}}_{t} \boldsymbol{A}_{t}^{H^{\prime}}=\mathbf{0}$, so that $\boldsymbol{A}_{t}^{H} \boldsymbol{Z}_{t}=\mathbf{0}$, see ? and ?. Moreover by choosing $\boldsymbol{C}_{t}$ such that $\boldsymbol{C}_{t} \boldsymbol{C}_{t}^{\prime}=$ $\boldsymbol{Z}_{t}^{\prime} \boldsymbol{\Sigma}_{\omega}^{-1} \boldsymbol{Z}_{t}$ and premultiplying the measurement equation by $\boldsymbol{A}_{t}$, we get:

$$
\left(\begin{array}{c}
\boldsymbol{A}_{t}^{L} \boldsymbol{y}_{t} \\
\boldsymbol{A}_{t}^{H} \boldsymbol{y}_{t}
\end{array}\right)=\left(\begin{array}{c}
\boldsymbol{A}_{t}^{L} \boldsymbol{c}_{t} \\
\boldsymbol{A}_{t}^{H} \boldsymbol{c}_{t}
\end{array}\right)+\left(\begin{array}{c}
\boldsymbol{C}_{t}^{\prime} \boldsymbol{Z}_{t} \\
\mathbf{0}
\end{array}\right) \boldsymbol{\alpha}_{t}+\left(\begin{array}{c}
\boldsymbol{A}_{t}^{L} \boldsymbol{G}_{t} \boldsymbol{\omega}_{t} \\
\boldsymbol{A}_{t}^{H} \boldsymbol{G}_{t} \boldsymbol{\omega}_{t}
\end{array}\right), \quad\left(\begin{array}{c}
\boldsymbol{A}_{t}^{L} \boldsymbol{G}_{t} \boldsymbol{\omega}_{t} \\
\boldsymbol{A}_{t}^{H} \boldsymbol{G}_{t} \boldsymbol{\omega}_{t}
\end{array}\right) \sim \mathrm{N}\left(\mathbf{0}\left(\begin{array}{cc}
\boldsymbol{I} & \mathbf{0} \\
\mathbf{0} & \boldsymbol{A}_{t}^{H} \widetilde{\boldsymbol{\Sigma}}_{t} \boldsymbol{A}_{t}^{H^{\prime}}
\end{array}\right)\right) .
$$

The unobserved factors are then calculated by applying the KFS to the first block of equations that depends on $\boldsymbol{\alpha}_{t}$. It is clear that this reduction technique speeds up signal extraction quite dramatically.

This computational device can be adapted to our problem. The reduction is carried out only for the monthly series. When there are no missing values, this leads to the following formulation:

$$
\left(\begin{array}{c}
\boldsymbol{Y}_{1 t}^{c} \\
A^{L} \Delta \boldsymbol{y}_{t} \\
A^{H} \Delta \boldsymbol{y}_{t}
\end{array}\right)=\left(\begin{array}{c}
\mathbf{0} \\
A_{t}^{L} \boldsymbol{c}_{t} \\
A_{t}^{H} \boldsymbol{c}_{t}
\end{array}\right)+\left(\begin{array}{c}
\boldsymbol{Z}_{1} \\
A_{t}^{L} \boldsymbol{Z}_{2} \\
\mathbf{0}
\end{array}\right) \boldsymbol{\alpha}_{t}+\left(\begin{array}{c}
\mathbf{0} \\
A_{t}^{L} \boldsymbol{\epsilon}_{2 t} \\
A_{t}^{H} \boldsymbol{\epsilon}_{2 t}
\end{array}\right)
$$

We cannot apply the reduction to observations that are missing at both time $t$ and time $t-1$; moreover, we do not apply the reduction to case 3 in the previous section, as it does not lead to significant computational gains.

\section{Estimation of the hyperparameters using the EM algorithm}

Maximum likelihood estimation of the model is carried out by the EM algorithm (?, ?). Let $\boldsymbol{y}_{j}=\left[\boldsymbol{y}_{j 0}^{\prime}, \ldots, \boldsymbol{y}_{j n}^{\prime}\right]^{\prime}, j=1,2$, in accordance with the partitioning made in Section 4 , and $\Delta \boldsymbol{y}_{j}=$ $\left[\Delta \boldsymbol{y}_{j 1}^{\prime}, \ldots, \Delta \boldsymbol{y}_{j n}^{\prime}\right]^{\prime}, j=1,2$; only $\boldsymbol{y}_{2}$ is observed at the monthly frequency. In our application, the complete data likelihood is the joint density of the observed $\Delta \boldsymbol{y}_{2}$, the missing data $\Delta \boldsymbol{y}_{1}$, and $\boldsymbol{f}=\left[\boldsymbol{f}_{0}^{\prime}, \ldots, \boldsymbol{f}_{n}^{\prime}\right]^{\prime}$

$$
g\left(\Delta \boldsymbol{y}_{1}, \Delta \boldsymbol{y}_{2}, \boldsymbol{f} ; \boldsymbol{\Xi}\right)=g\left(\Delta \boldsymbol{y}_{1} \mid \boldsymbol{f} ; \boldsymbol{\Xi}\right) g\left(\Delta \boldsymbol{y}_{2} \mid \boldsymbol{f} ; \boldsymbol{\Xi}\right) g(\boldsymbol{f} ; \boldsymbol{\Xi})
$$


Defining the quasi-differences

$$
w_{i t}= \begin{cases}\sqrt{1-\psi_{i}^{2}} \Delta y_{i t}, & t=1 \\ \Delta y_{i t}-\psi_{i} \Delta y_{i, t-1}, & t=2, \ldots, n\end{cases}
$$

and

$$
\boldsymbol{x}_{i t}= \begin{cases}\sqrt{1-\psi_{i}^{2}}\left[1, \boldsymbol{f}_{t}^{\prime}\right]^{\prime}, & t=1, \\ {\left[\left(1-\psi_{i}\right),\left(\boldsymbol{f}_{t}-\psi_{i} \boldsymbol{f}_{t-1}\right)^{\prime}\right]^{\prime},} & t=2, \ldots, n\end{cases}
$$

we can write

$$
\ln g\left(\Delta \boldsymbol{y}_{j} \mid \boldsymbol{f} ; \boldsymbol{\Xi}\right)=-\frac{1}{2} \sum_{i=1}^{N_{j}}\left\{n \ln \sigma_{i}^{2}+\frac{1}{\sigma_{i}^{2}} \sum_{t=1}^{n}\left(w_{i t}-\boldsymbol{x}_{i t}^{\prime} \boldsymbol{m}_{i}\right)^{2}\right\}
$$

where

$$
\boldsymbol{m}_{i}=\left[\begin{array}{c}
\delta_{i} \\
\boldsymbol{\theta}_{i}
\end{array}\right]
$$

This result arises because, for the $i$ th series,

$$
\Delta y_{i t}=\mu_{i}+\boldsymbol{f}_{t}^{\prime} \boldsymbol{\theta}_{i}+u_{i t}, \quad u_{i t}=\psi_{i} u_{i, t-1}+\epsilon_{i t},
$$

with $\epsilon_{i t} \sim \operatorname{NID}\left(0, \sigma_{i}^{2}\right)$. Hence, $\Delta y_{i t}, i=1, \ldots, N$, are conditionally independent given $\boldsymbol{f}_{t}$, and are characterised by errors that follow a first order AR model. As a result, if a Choleski orthogonalisation is performed, we get Eq. (17). Moreover,

$$
\begin{aligned}
\ln g(\boldsymbol{f} ; \boldsymbol{\Xi})= & -\frac{1}{2}\left[n \ln \left|\boldsymbol{\Sigma}_{\eta}\right|+\operatorname{tr}\left\{\boldsymbol{\Sigma}_{\eta}^{-1} \sum_{t=1}^{n}\left(\boldsymbol{f}_{t}-\boldsymbol{\Phi} \boldsymbol{f}_{t-1}\right)\left(\boldsymbol{f}_{t}-\boldsymbol{\Phi} \boldsymbol{f}_{t-1}\right)^{\prime}\right\}\right] \\
& -\frac{1}{2}\left[\ln \left|\boldsymbol{P}_{0}\right|+\operatorname{tr}\left\{\boldsymbol{P}_{0}^{-1} \boldsymbol{f}_{0} \boldsymbol{f}_{0}^{\prime}\right\}\right]
\end{aligned}
$$

where $\boldsymbol{P}_{0}$ satisfies the matrix equation $\boldsymbol{P}_{0}=\boldsymbol{\Phi} \boldsymbol{P}_{0} \boldsymbol{\Phi}^{\prime}+\boldsymbol{\Sigma}_{\eta}$. The restrictions imposed on the factor structure imply:

$$
\ln g(\boldsymbol{f} ; \boldsymbol{\Xi})=-\frac{1}{2} \sum_{k=1}^{K}\left\{n+\sum_{t=1}^{n}\left(f_{k t}-\phi_{k} f_{k, t-1}\right)^{2}-\ln \left(1-\phi_{k}^{2}\right)+\frac{f_{k 0}^{2}}{1-\phi_{k}^{2}}\right\} .
$$

Given an initial parameter value, $\boldsymbol{\Xi}^{*}$, the EM algorithm maximizes the intermediate quantity (?) iteratively with respect to $\Xi$ :

$$
\begin{aligned}
Q\left(\boldsymbol{\Xi} ; \boldsymbol{\Xi}^{*}\right) & =\mathrm{E}_{\Xi^{*}}\left[\ln g\left(\Delta \boldsymbol{y}_{1}, \Delta \boldsymbol{y}_{2}, \boldsymbol{f} ; \boldsymbol{\Xi}\right)\right] \\
& =\int \ln g\left(\Delta \boldsymbol{y}_{1}, \Delta \boldsymbol{y}_{2}, \boldsymbol{f} ; \boldsymbol{\Xi}\right) g\left(\Delta \boldsymbol{y}_{1}, \boldsymbol{f} \mid \Delta \boldsymbol{y}_{2}, \boldsymbol{Y}_{1}^{c} ; \boldsymbol{\Xi}^{*}\right) d\left(\Delta \boldsymbol{y}_{1}, \boldsymbol{f}\right),
\end{aligned}
$$

which is interpreted as the expectation of the complete data log-likelihood with respect to $g\left(\Delta \boldsymbol{y}_{1}, \boldsymbol{f} \mid\right.$ $\left.\Delta \boldsymbol{y}_{2}, \boldsymbol{Y}_{1}^{c} ; \boldsymbol{\Xi}^{*}\right)$, which is the conditional probability density function of the unobservable states, given the observations, evaluated at $\boldsymbol{\Xi}^{*}$.

? show that the parameter estimates that maximise the $\log$-likelihood $\log L(\boldsymbol{\Xi})$ can be obtained by a sequence of iterations, each consisting of an expectation step (E-step) and a maximization step (M-step), that aim to locate a stationary point of $Q\left(\boldsymbol{\Xi} ; \boldsymbol{\Xi}^{*}\right)$. 


\subsection{The E-step}

At iteration $m$, given the estimate $\boldsymbol{\Xi}^{(m)}$, the E-step deals with the evaluation of $Q\left(\boldsymbol{\Xi} ; \boldsymbol{\Xi}^{(m)}\right)$; this is carried out with the support of the KFS applied to the state space representation provided in Section 4.2 with hyperparameters $\boldsymbol{\Xi}^{(m)}$.

As is evident from Eqs. (17)- (19) the E-step computes

$$
\begin{gathered}
\mathrm{E}_{\Xi^{(m)}}\left(\sum_{t=1}^{n} \boldsymbol{x}_{i t} \boldsymbol{x}_{i t}^{\prime}\right), \quad \mathrm{E}_{\Xi^{(m)}}\left(\sum_{t=1}^{n} \boldsymbol{x}_{i t} w_{i t}\right), \quad \mathrm{E}_{\Xi^{(m)}}\left(\sum_{t=1}^{n} w_{i t}^{2}\right), \\
\mathrm{E}_{\Xi^{(m)}}\left(\sum_{t=2}^{n} f_{k, t-1}^{2}\right), \quad \mathrm{E}_{\Xi^{(m)}}\left(\sum_{t=1}^{n} f_{k t}^{2}\right), \mathrm{E}_{\Xi^{(m)}}\left(\sum_{t=2}^{n} f_{k t} f_{k, t-1}\right),
\end{gathered}
$$

where the expectation is taken with respect to the density $g\left(\Delta \boldsymbol{y}_{1}, \boldsymbol{f} \mid \Delta \boldsymbol{y}_{2}, \boldsymbol{Y}_{1}^{c} ; \boldsymbol{\Xi}^{(m)}\right)$. As a byproduct,

$$
\mathrm{E}_{\Xi^{(m)}}\left(\sum_{t=2}^{n} u_{i, t-1}^{2}\right), \mathrm{E}_{\Xi^{(m)}}\left(\sum_{t=1}^{n} u_{i t}^{2}\right), \mathrm{E}_{\Xi^{(m)}}\left(\sum_{t=2}^{n} u_{i t} u_{i, t-1}\right),
$$

are made available. It must be remarked that for $i=N_{1}+1, \ldots, N$, $w_{i t}$ is observed, and thus $\mathrm{E}_{\Xi^{(m)}}\left(\sum_{t} w_{i t}^{2}\right)=\sum_{t} w_{i t}^{2}, \mathrm{E}_{\Xi^{(m)}}\left(\sum_{t} \boldsymbol{x}_{i t} w_{i t}\right)=\sum_{t} \mathrm{E}_{\Xi^{(m)}} w_{i t}\left(\boldsymbol{x}_{i t}\right)$.

In order to be able to evaluate the covariance between the current and past states, e.g., $\mathrm{E}_{\Xi^{(*)}}\left(\sum_{t=2}^{n}\right.$ $\left.f_{k t} f_{k, t-1}\right)$, the KFS recursion needs to be augmented as per $?$ and $?$.

In summary, the E-step consists of evaluating the conditional expectation of cross-products of random quantities. For instance,

$$
\mathrm{E}_{\Xi^{(m)}}\left(\sum_{t=1}^{n} \boldsymbol{x}_{i t} \boldsymbol{x}_{i t}^{\prime}\right)=\sum_{t=1}^{n}\left[\tilde{\boldsymbol{x}}_{i t \mid n} \tilde{\boldsymbol{x}}_{i t \mid n}^{\prime}+\boldsymbol{V}_{x \mid n}\right]
$$

where $\tilde{\boldsymbol{x}}_{i t \mid n}=\mathrm{E}\left(\boldsymbol{x}_{i t} \mid \Delta \boldsymbol{y}_{2}, \boldsymbol{Y}_{1}^{c} ; \Xi^{(m)}\right)$ and $\boldsymbol{V}_{x \mid n}=\operatorname{Var}\left(\boldsymbol{x}_{i t} \mid \Delta \boldsymbol{y}_{2}, \boldsymbol{Y}_{1}^{c} ; \Xi^{(m)}\right)$.

\subsection{The M-step}

The M-step amounts to choosing a new value $\boldsymbol{\Xi}^{(m+1)}$ so as to maximize the criterion $Q\left(\boldsymbol{\Xi} ; \boldsymbol{\Xi}^{(m)}\right)$ with respect to $\boldsymbol{\Xi}$, i.e., $Q\left(\boldsymbol{\Xi}^{(m+1)} ; \boldsymbol{\Xi}^{(m)}\right) \geq Q\left(\boldsymbol{\Xi}^{(m)} ; \boldsymbol{\Xi}^{(m)}\right)$. The maximization is in closed form if, as is customary, we ignore the term $\ln \left(1-\phi_{k}^{2}\right)+\frac{f_{k 0}^{2}}{1-\phi_{k}^{2}}$ in Eq. 18$)$ and drop the component $\left(w_{i 1}-\boldsymbol{x}_{i 1}^{\prime} \boldsymbol{\delta}_{i}\right)^{2}$ for $t=1$ in Eq. (17).

As a result of this simplification, the new estimates of the means and the loadings of the $i$ th series, $i=1, \ldots, N_{1}$, are

$$
\left[\begin{array}{l}
\hat{\delta}_{i}^{(m+1)} \\
\hat{\boldsymbol{\theta}}_{i}^{(m+1)}
\end{array}\right]=\left[\sum_{t=1}^{n}\left(\tilde{\boldsymbol{x}}_{i t \mid n} \tilde{\boldsymbol{x}}_{i t \mid n}^{\prime}+\boldsymbol{V}_{x \mid n}\right)\right]^{-1} \sum_{t=1}^{n}\left(\tilde{\boldsymbol{x}}_{i t \mid n} \tilde{w}_{i t \mid n}+\boldsymbol{V}_{x w \mid n}\right),
$$

where $\tilde{w}_{i t \mid n}=\mathrm{E}\left(w_{i t} \mid \Delta \boldsymbol{y}_{2}, \boldsymbol{Y}_{1}^{c} ; \Xi^{(m)}\right)$ and $\boldsymbol{V}_{x w \mid n}=\operatorname{Cov}\left(\boldsymbol{x}_{i t}, w_{i t} \mid \Delta \boldsymbol{y}_{2}, \boldsymbol{Y}_{1}^{c} ; \Xi^{(m)}\right)$.

For the series belonging to the second group, $i=N_{1}+1, \ldots, N$,

$$
\left[\begin{array}{l}
\hat{\delta}_{i}^{(m+1)} \\
\hat{\boldsymbol{\theta}}_{i}^{(m+1)}
\end{array}\right]=\left[\sum_{t=1}^{n}\left(\tilde{\boldsymbol{x}}_{i t \mid n} \tilde{\boldsymbol{x}}_{i t \mid n}^{\prime}+\boldsymbol{V}_{x \mid n}\right)\right]^{-1}\left(\boldsymbol{I}-\boldsymbol{\Delta}_{t}\right) \sum_{t=1}^{n} \tilde{\boldsymbol{x}}_{i t \mid n} w_{i t}+\boldsymbol{\Delta}_{t} \sum_{t=1}^{n} \tilde{\boldsymbol{x}}_{i t \mid n} \boldsymbol{V}_{x m \mid n}
$$


where $\boldsymbol{I}$ is a unitary matrix and $\boldsymbol{\Delta}_{t}$ is a matrix with element $\Delta_{i, j}=1$ when an observation is missing. Finally, $\boldsymbol{V}_{x m \mid n}$ is the smooth covariance between missing values that has to be calculated for the maximization step.

Since $w_{i t}$ is observed,

$$
\hat{\sigma}_{i}^{(m+1)}=\frac{1}{n} \sum_{t=1}^{n} \mathrm{E}_{\Xi(m)}\left[\left(w_{i t}-\boldsymbol{x}_{i t}^{\prime} \boldsymbol{\delta}_{i}^{(m+1)}\right)^{2}\right]
$$

where, if $i \leq N_{1}$,

$$
\mathrm{E}_{\Xi^{(m)}}\left[\left(w_{i t}-\boldsymbol{x}_{i t}^{\prime} \boldsymbol{\delta}_{i}^{(m)}\right)^{2}\right]=\left(\tilde{w}_{i t \mid n}-\tilde{\boldsymbol{x}}_{i t \mid n}^{\prime} \boldsymbol{\delta}_{i}^{(m)}\right)^{2}+V_{e \mid n}
$$

and $V_{e \mid n}=\operatorname{Var}\left(w_{i t}-\boldsymbol{x}_{i t}^{\prime} \boldsymbol{\delta}_{i}^{(m)} \mid \Delta \boldsymbol{y}_{2}, \boldsymbol{Y}_{1}^{c} ; \Xi^{(m)}\right)$; otherwise, $w_{i t}$ is available, and thus,

$$
\mathrm{E}_{\Xi^{(m)}}\left[\left(w_{i t}-\boldsymbol{x}_{i t}^{\prime} \boldsymbol{\delta}_{i}^{(m)}\right)^{2}\right]=\left(w_{i t}-\tilde{\boldsymbol{x}}_{i t \mid n}^{\prime} \boldsymbol{\delta}_{i}^{(m)}\right)^{2}+V_{e \mid n} .
$$

The estimates of the factor autoregressive coefficients are

$$
\hat{\phi}_{k}^{(m+1)}=\frac{\sum_{t=1}^{n}\left(\tilde{f}_{k t \mid n} \tilde{f}_{k, t-1 \mid n}+V_{f f_{1} \mid n}\right)}{\sum_{t=1}^{n}\left(\tilde{f}_{k, t-1 \mid n}^{2}+V_{f_{1} f_{1} \mid n}\right)},
$$

where $\tilde{f}_{k t \mid n}=\mathrm{E}\left(f_{k t} \mid \Delta \boldsymbol{y}_{2}, \boldsymbol{Y}_{1}^{c} ; \Xi^{(m)}\right), V_{f f_{1} \mid n}=\operatorname{Cov}\left(f_{k t}, f_{k, t-1} \mid \Delta \boldsymbol{y}_{2}, \boldsymbol{Y}_{1}^{c} ; \Xi^{(m)}\right)$, and $V_{f_{1} f_{1} \mid n}=$ $\operatorname{Var}\left(f_{k, t-1} \mid \Delta \boldsymbol{y}_{2}, \boldsymbol{Y}_{1}^{c} ; \Xi^{(m)}\right)$.

As for the parameters of the idiosyncratic components,

$$
\hat{\psi}_{i}^{(m+1)}=\frac{\sum_{t=2}^{n}\left(\tilde{u}_{i t \mid n} \tilde{u}_{i, t-1 \mid n}+V_{u u_{1} \mid n}\right)}{\sum_{t=2}^{n}\left(\tilde{u}_{i, t-1 \mid n}^{2}+V_{u_{1} u_{1} \mid n}\right)},
$$

where $\tilde{u}_{i t \mid n}=\mathrm{E}\left(u_{i t} \mid \Delta \boldsymbol{y}_{2}, \boldsymbol{Y}_{1}^{c} ; \Xi^{(m)}\right), V_{u u_{1} \mid n}=\operatorname{Cov}\left(u_{i t}, u_{i, t-1} \mid \Delta \boldsymbol{y}_{2}, \boldsymbol{Y}_{1}^{c} ; \Xi^{(m)}\right)$, and $V_{u_{1} u_{1} \mid n}=$ $\operatorname{Var}\left(u_{i, t-1} \mid \Delta \boldsymbol{y}_{2}, \boldsymbol{Y}_{1}^{c} ; \Xi^{(m)}\right)$.

When $\Delta \boldsymbol{y}_{2 t}$ is subject to missing values, the algorithm has to be modified so as to take into account the conditional covariances between the missing values and the latent states.

\section{Description of the dataset}

We now apply the methodology described in the previous sections to the construction of Euromind-C, an indicator of economic conditions in the Euro area and its largest member states. The set of time series making up the system has dimension $N=171$. The first 55 are the quarterly national accounts series concerning the value added chained volumes with reference year 2000 for all of the sectors and the main expenditure components of the GDP, referring to the Euro area and the four selected countries (see section 11). The remaining time series are monthly indicators.

We have carried out a systematic review of data availability by geographical entity, which has led to the recognition of the presence of various important gaps in the information set: the production of the new orders indicator in industry has been discontinued; the collection of new car registration data has been discontinued by Eurostat, while the series continue to be made available by other sources; and some labour market indicators in short term business statistics are no longer produced regularly. Nevertheless, 
since our methods allow for arbitrary patterns of missing values, we decided to retain the series in the database, even if they are out of date, in order to support the historical disaggregation of the value added.

Table 4 provides a list of the time series that have been used in the estimation of EuroMInd-C, organized by GDP component. The series are organized according to the reference sector or GDP component:

- Agriculture: the monthly indicators concern the production of milk, bovine meat production in tons, pigs meat production in tons.

- Industry: the monthly indicators concern the index of industrial production, the index of turnover, and new orders; we also include the industrial confidence indicator compiled by the European Commission.

- Construction: the monthly indicators concern the volume index of production for construction, employment and hours worked (Germany), and building permits.

- Trade, transport and communication: the monthly indicators concern the total turnover of the retail sector, the index of deflated turnover, employment (Germany) and car registrations, along with the survey-based retail confidence indicator.

- Financial services and business: the monthly indicators concern the index of industrial production as a proxy measure of the state of the economy, the stock market indices and capitalization (when available), a set of five monetary aggregates for the Euro area (M1-M3, loans, etc.), and financial ones (deposits of residents held at monetary financial institutions, and credit to total residents granted by monetary financial institutions).

- Other services: the monthly indicators concern the index of industrial production, the index of retail turnover, and the monthly unemployment rate as proxy measures of the state of the economy.

- Taxes less subsidies on products: the monthly indicators concern the index of industrial production and the index of retail turnover as proxy measures of the state of the economy.

- Final consumption expenditures: the monthly indicators concern the index of deflated turnover of the retail sector, car registrations, and two survey indicators, the index of consumer confidence and the index of major purchases at present, compiled by the European Commission.

- Gross capital formation: the monthly indicators concern the index of industrial production for capital goods and the index of economic sentiment compiled by the European Commission.

- Exports of goods and services: the monthly indicators concern the index of industrial production and the volume index of exports.

- Imports of goods and services: the monthly indicators concern the index of industrial production and the volume index of imports.

For a complete list of the series in the dataset and their reference periods, see Appendix A.

\section{Estimation results}

The dynamic factor model is estimated for the sample period January 1995-September 2012. The computational complexity of the model is quite substantial, with a convergence of the EM algorithm after 250 iterations. Conditional on the maximum likelihood estimates of the hyperparameters, we can perform signal extraction according to the nonlinear smoothing algorithm outlined in the previous sections. 

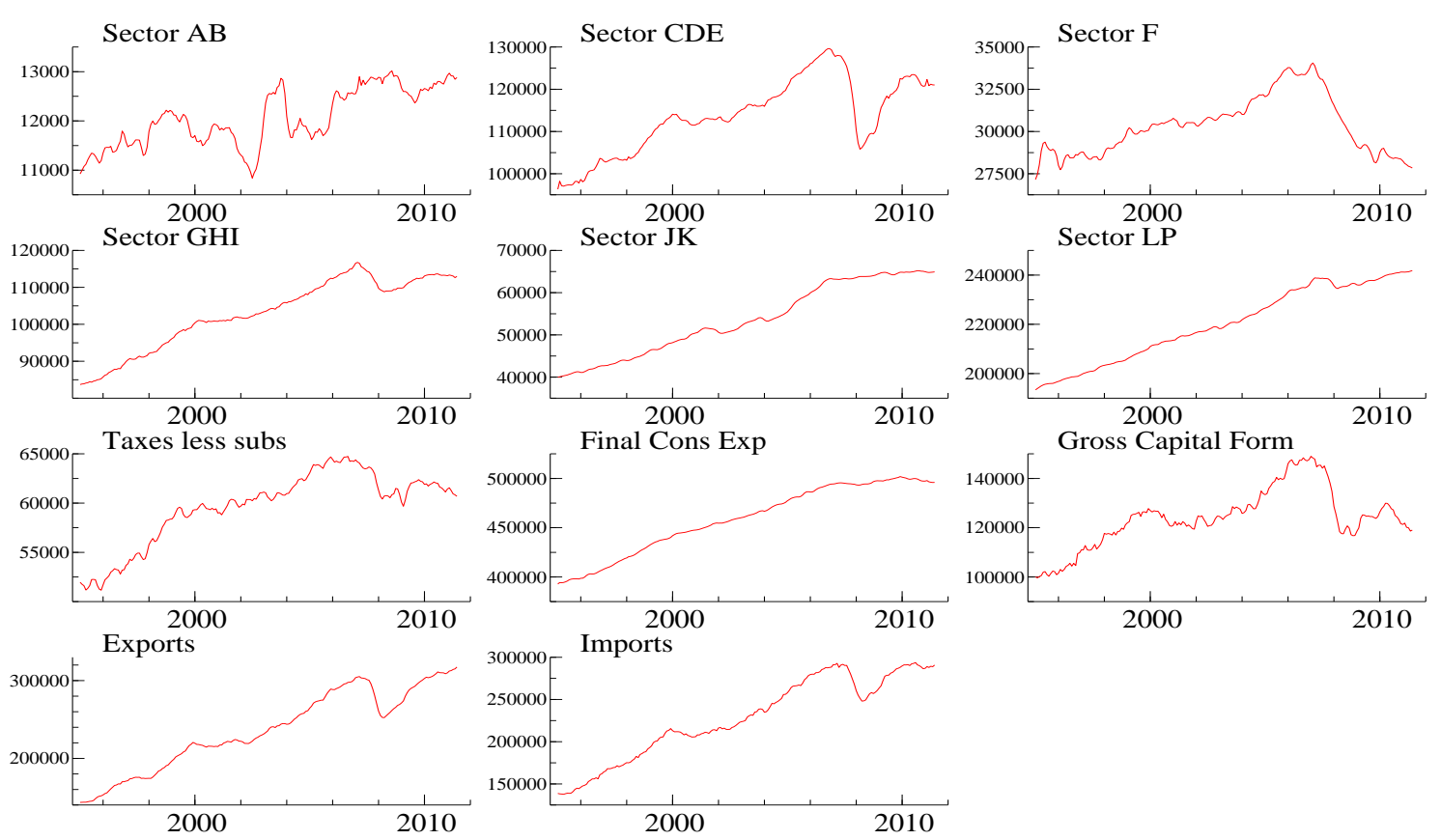

Figure 1: Monthly indicators of the 11 components of Euro area GDP, levels (chained 2000 volumes).

This yields the monthly estimates of the components of GDP (disaggregated also by country) by economic activity and expenditure components, from which the estimates of total GDP at market prices are compiled, according to the contemporaneous aggregation procedure outlined in Appendix C.

EuroMInd-C makes available the monthly estimates of the 11 GDP components for the Euro area and its four largest economies; these are relevant for the assessment of the state of the economy from the point of view of sectorial value added and by expenditure component.

Figure 1 displays the levels in millions of chained euros, whereas Figure 2 presents the growth rates for the Euro area as a whole. It is noticeable that the real effects of the global financial crisis hit the manufacturing sector quite dramatically, although the recovery was sustained. The effects of the fall on the output of the construction sector have been prolonged, and, by the end of 2012, there had been no exit from the recession for this sector.

\subsection{The reconciliation of the estimates of GDP from the output and the expenditure approach}

As was stated in Section 4 the dataset for each of the four countries and the Euro area consists of the 11 quarterly GDP components. Our model produces disaggregate estimates of the monthly components conditional on the information available, and the estimates can then be combined to produce the final estimate of monthly GDP. Two issues are posed by the contemporaneous aggregation of the output and expenditure components of GDP. First and foremost, the quarterly national account series are subject to the accounting deterministic constraints

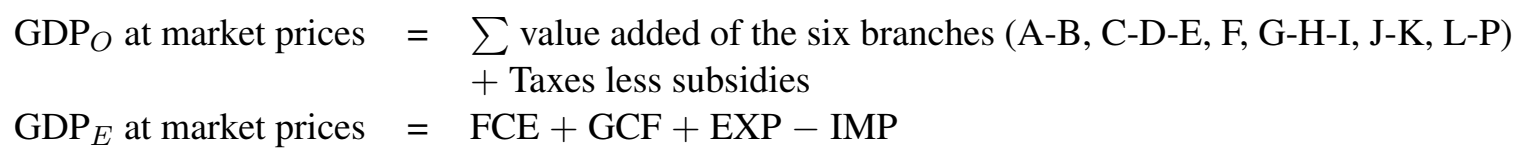



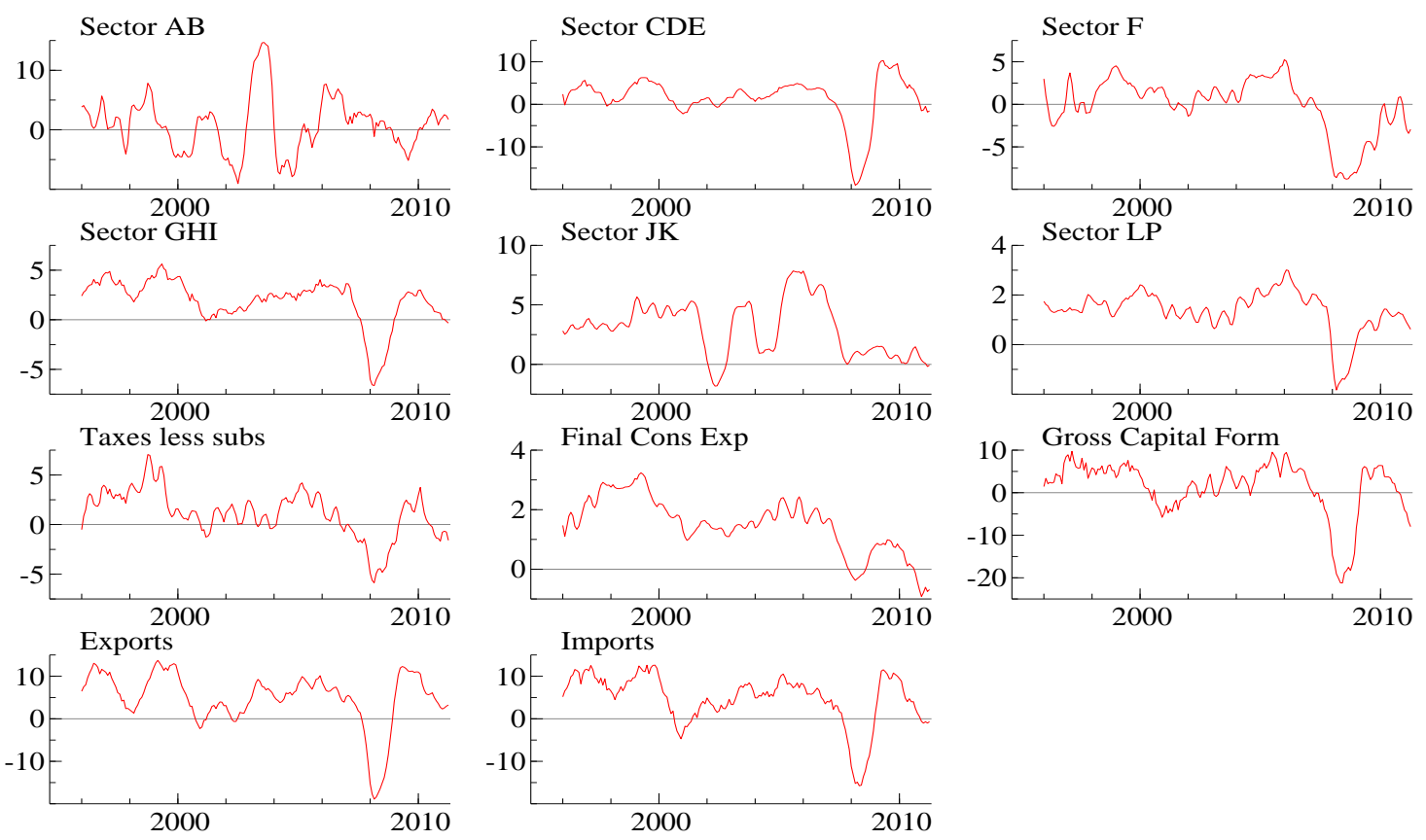

Figure 2: Monthly indicators of the 11 components of Euro area GDP, yearly growth rates (chained 2000 volumes).

only when the aggregates are expressed at current prices and at the average prices of the previous year. Secondly, the values of GDP at market prices from the output and expenditure approaches do not necessarily coincide, and therefore have to be reconciled.

The first issue is a consequence of the current method of producing chained linked national accounts estimates. Chaining, which is a multiplicative operation, prevents the national accounts constraints from being additive, so that a nonzero discrepancy arises. GDP and its main components are expressed in chain-linked volumes (millions of euros), with a reference year of 2000, which implies that the constraints only hold exactly for the four quarters of the year 2001.

Interestingly, due to the application of the annual overlap technique for the compilation of the quarterly national accounts volume data, constraints are not lost entirely, but continue to hold after a transformation of the data, which we refer to as "dechaining" and which aims to express the chained values at the previous year's prices. According to the annual overlap technique (see ?, Ch. IX), the estimates for each quarter are compiled using the weighted annual average prices of the previous year; this produces quarterly volume estimates that sum to exactly the corresponding annual aggregate. As a result, the disaggregated (monthly and quarterly) volume measures expressed at the previous year's prices preserve both the temporal and cross-sectional additivity.

The cross-sectional constraints can be enforced by a multistep procedure that de-chains the estimated monthly values, expressing them at the average prices of the previous year, and projects the estimates on the subspace of the constraints. The procedure is described in detail by ? and reproduced in Appendix D.

The monthly GDP estimates for the Euro area and the four largest economies that arise from the output approach are displayed in Figure 3, together with their approximate 95\% confidence limits.

The confidence intervals take into account what is often referred to as filtering uncertainty, i.e., they reflect the estimation error variance of the unobserved monthly time series, conditional on the full avail- 

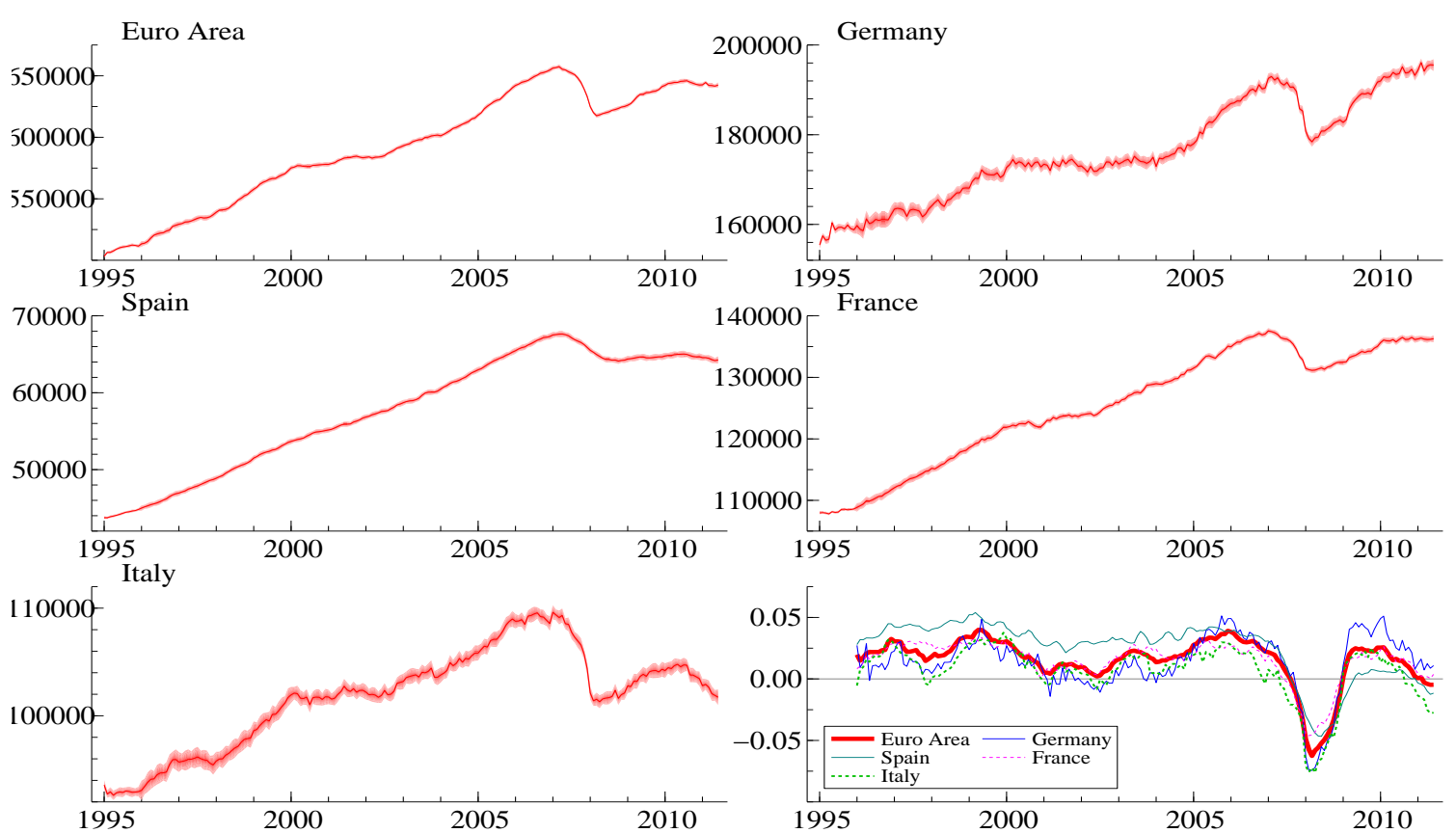

Figure 3: EuroMInd-C estimates: Monthly indicator of GDP at market prices (chained 2000 volumes). Estimates from the output approach.

able observed sample and the maximum likelihood estimates of the parameters. Hence, the confidence regions do not reflect the parameter uncertainty.

It should be noted that the mean square estimation error of the unobserved monthly series is higher for Germany and Italy; this is because the two countries have higher shares of value added of the manufacturing sector (branch C-D-E), which is more volatile than the other sectors (excluding agriculture). The bottom right panel displays the underlying yearly growth rates.

The estimates from the expenditure approach are obtained from the sum of consumption, investment and net exports (FCE + GCF + EXP - IMP). They are displayed in Figure 4 along with their 95\% confidence intervals and the underlying yearly growth rates (bottom right panel).

As is well known, the estimates arising from the output and expenditure approaches are not coincident; however, they differ only slightly and can be reconciled by a suitable combination, which is carried out as follows. Denoting the GDP estimates obtained using the output approach by $Y_{t}^{o}$, those from the expenditure side by $Y_{t}^{e}$, and the estimation error variance of the output and expenditure estimates by $S_{t}^{2 o}$ and $S_{t}^{2 e}$, we use a set of weights proportional to the relative precision of the output estimates, that is

$$
w_{o t}=\frac{1 / S_{t}^{2 o}}{1 / S_{t}^{2 o}+1 / S_{t}^{2 e}} .
$$

The combined estimates are then balanced according to the procedure outlined in Appendix C. The combined estimates of the yearly and monthly growth rates are displayed in Figure 5. Obviously, it turns out that the combined estimate is more precise than either $Y_{t}^{o}$ or $Y_{t}^{e}$. The last panel illustrates quite effectively the average and differential pace at which the recovery took place after the global financial crisis, and the depth of the recessionary movement.

Figure 6 provides a comparison of the estimates of the yearly growth rates that arise from the expenditure and output approaches, and also superimposes the final Euromind-C estimates of the growth rates. The 

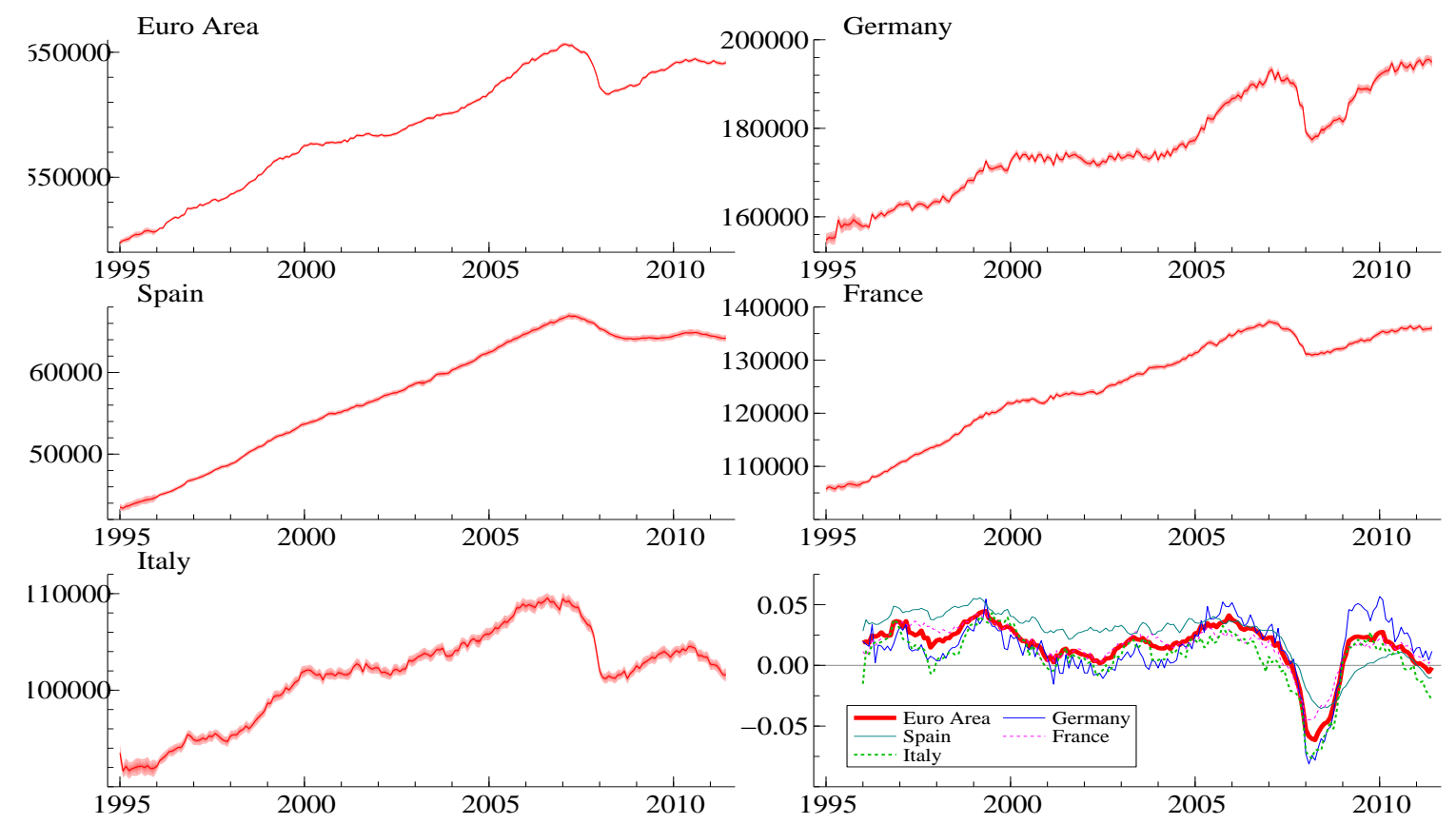

Figure 4: EuroMInd-C estimates: Monthly indicator of GDP at market prices (chained 2000 volumes). Estimates from the expenditure approach.
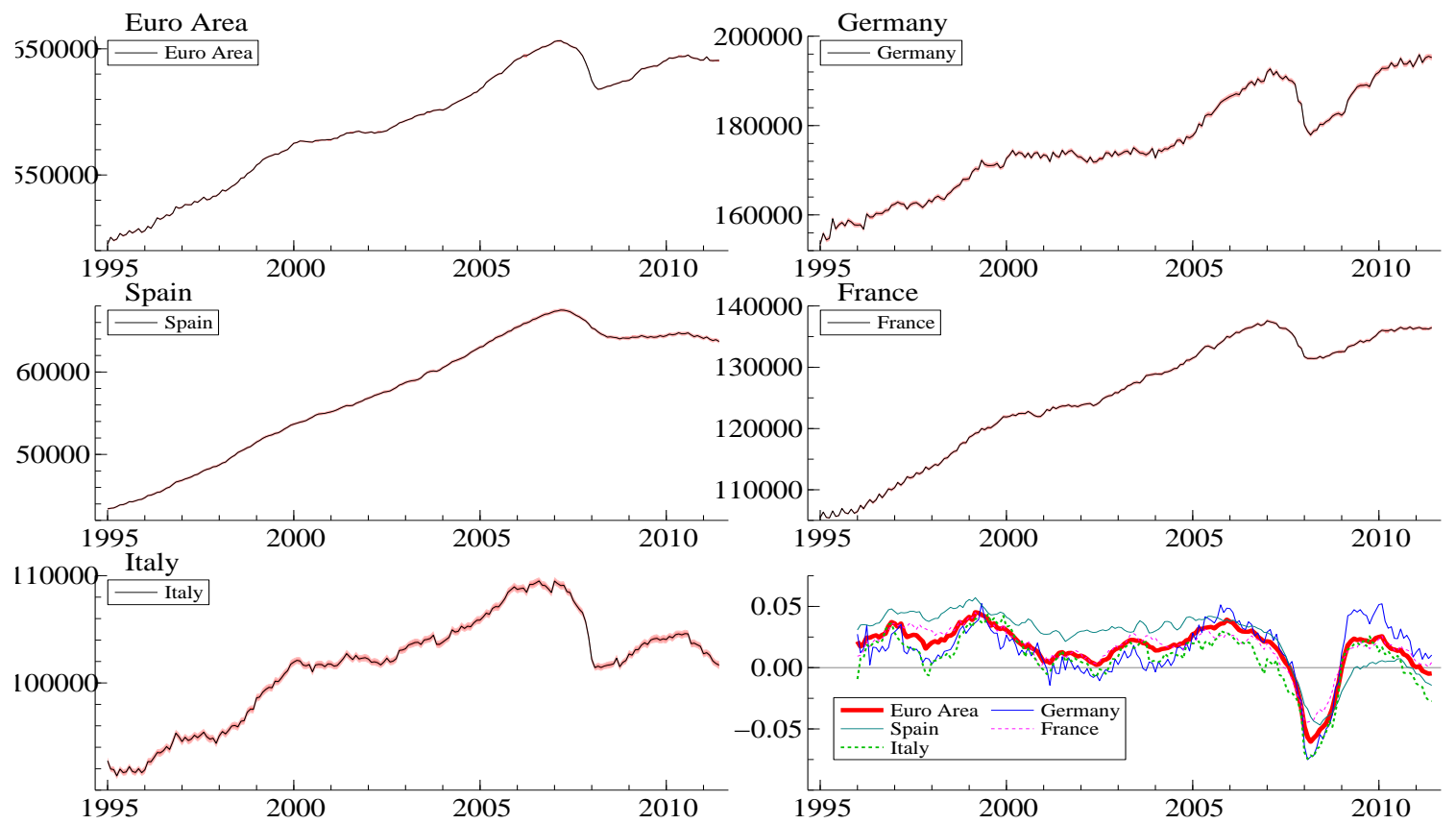

Figure 5: EuroMInd-C: GDP at market prices (chained 2000 volumes). Combined estimates. 

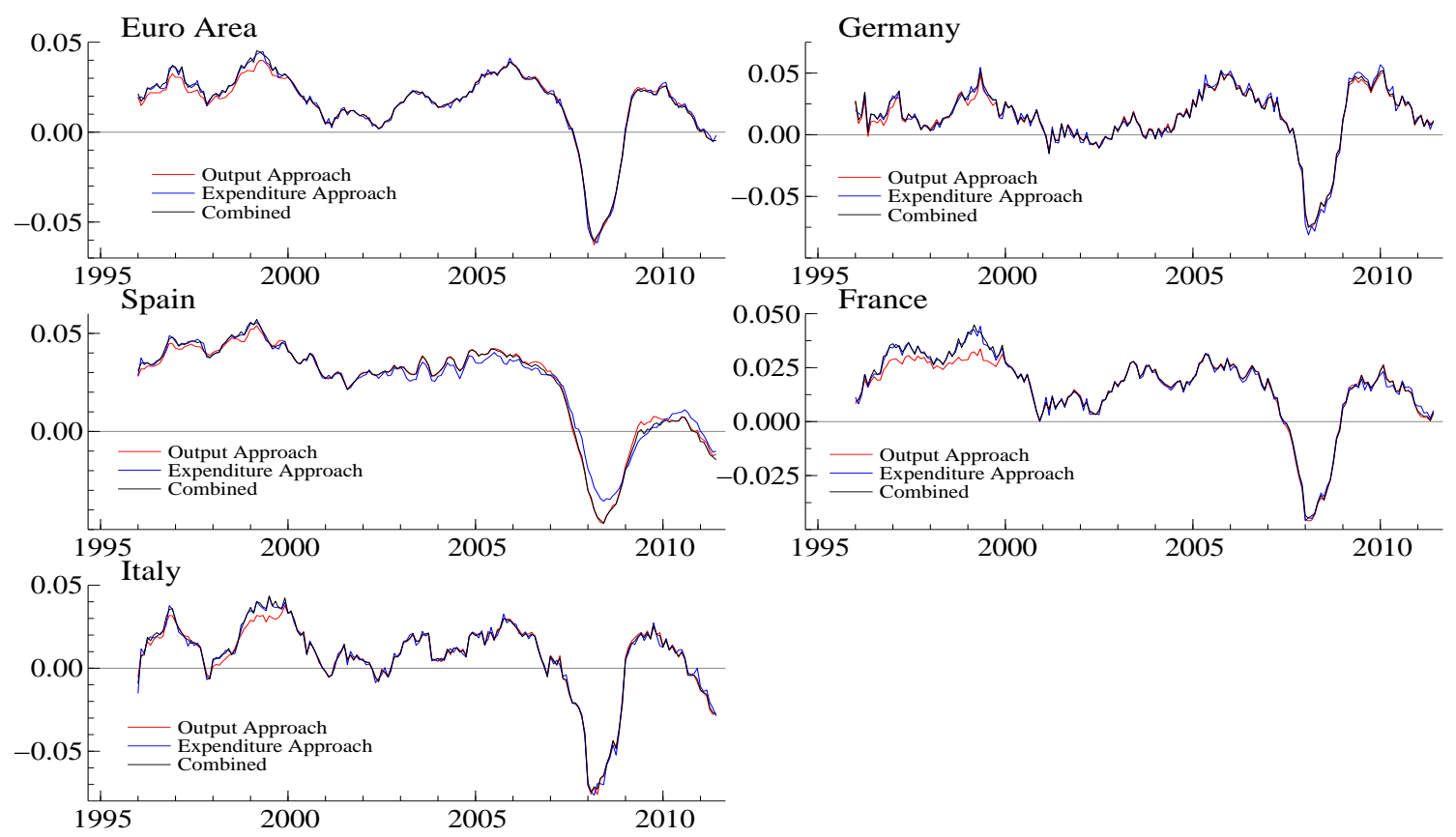

Figure 6: EuroMInd-C estimates: Comparison of the estimates of the yearly growth rates that arise from the expenditure and output approaches.

most sizable differences concern the growth rates for Spain, and relate to the statistical discrepancy between the quarterly estimates of GDP at market prices obtained from the output and expenditure approaches. As such, the differences are due to the characteristics of the raw data, rather than of our methodology. In general, the estimates arising from the expenditure approach are more volatile.

\subsection{Variance decompositions}

The monthly indicator of GDP draws information from the latent factors. Each variable (monthly indicators and national account series) contributes to the definition of the factors, according to the model specification. Ceteris paribus, it is desirable that the individual variables load significantly on the common factors and that the latter contribute significantly to the national account series. For country $c=2, \ldots, C$, the relative contributions of the factors in explaining the variability of the series can be obtained from the following variance decomposition:

$$
\operatorname{Var}\left(\Delta y_{c s, t}\right)=\theta_{c s, 0}^{2} \operatorname{Var}\left(\Delta \mu_{t}\right)+\theta_{c s, 1}^{2} \operatorname{Var}\left(\Delta \mu_{c t}\right)+\theta_{c s, 2}^{2} \operatorname{Var}\left(\Delta \mu_{s t}\right)+\operatorname{Var}\left(\Delta \mu_{c s, t}^{*}\right) .
$$

For the Euro area,

$$
\operatorname{Var}\left(\Delta y_{1 s, t}\right)=\theta_{1 s, 0}^{2} \operatorname{Var}\left(\Delta \mu_{t}\right)+\sum_{c=2}^{C} \theta_{1 c, 1}^{2} \operatorname{Var}\left(\Delta \mu_{c t}\right)+\theta_{1 s, 2}^{2} \operatorname{Var}\left(\Delta \mu_{s t}\right)+\operatorname{Var}\left(\Delta \mu_{1 s, t}^{*}\right) .
$$

The four addends on the right, expressed as a percentage of the total variance on the left hand side, are reported in Table 2, which displays the median value (middle columns) and the 33th (1/3) and 66 $(2 / 3)$ percentiles of the sampling distribution of the statistic. The table is divided into separate panels, referring to the different series, with the first 11 referring to the national account series. The following panels refer to the different groups of monthly indicators. When interpreting these numbers, it has to be 
taken into account that the larger the variance share that is absorbed by the common and sector-specific factors, the greater the contribution of an indicator to EuroMind-C.

An interesting fact is that the value added of the sectors C-D-E (manufacturing), G-H-I (trade, transport and communication services) and J-K (financial services and business activities) display the highest degree of commonality, whereas construction and other services are predominantly idiosyncratic. As far as the expenditure components are concerned, comovements play a larger role for GCF, whereas the other components (consumption, exports and imports) are predominantly idiosyncratic. This may be the reason why the EuroMInd-C estimates of GDP according to the expenditure approach are less reliable than those arising from the output approach.

Another conclusion that we may draw from Table 2 is that the Spanish economy behaves more idiosyncratically. Of the monthly indicators, the industrial production series (sts_impr_msa) contribute most sizably to the common and component-specific factors. Similar considerations hold for industrial turnover and deflated turnover of the retail sector (sts_trtu_defltv).

The least significant contributions to the common factors are associated with the indicators for the construction sector (sts_colb and sts_copr), the financial indicators (mfma_m), the survey indicators for the expenditure components (bssi_FCE), and the sector G-H-I (sbs_ciGHI). One possible use of this information is the deletion of series that do not contribute significantly to the common factors.

\section{A pseudo real-time comparative assessment of Euromind-C}

We now provide a comparative assessment of EuroMInd-C by performing a recursive forecasting experiment that focuses on its ability to predict GDP and its components for the Euro area, referring to quarters $\tau=2005 . q 1,2005 . q 2, \ldots, 2011 . q 4$, using the information set that is available at the end of the month preceding the end of the quarter (February 2005 for 2005.q1, May 2005 for 2005.q2, etc.). The prediction of the 11 components of GDP arises from the aggregation of the nowcasts for the estimation month and the previous month (e.g., February 2005 and January 2005 when predicting 2005.q1) and the one-step-ahead forecast for the last month of the quarter (March 2005). The model is re-estimated every time using the most recent data.

The same experiment is carried out for EuroMInd, which uses the componentwise estimation methodology described by Frale et al. (2010). This yields $M=28$ forecasts $\hat{Y}_{i \tau}$, where $i$ indexes a particular GDP component and $\tau=2005 . q 1, \ldots 2011 . q 4$, for each method.

We also consider as a reference the naïve ("no change" or random walk) predictor $\hat{Y}_{i \tau}=Y_{i, \tau-1}$, which will be referred to as RW henceforth. We expect the EuroMInd and EuroMInd-C predictions to be more informative, as they exploit the monthly information made available for the first two months of the quarter.

For the three predictors, we compare the forecasts with the observed value, $Y_{i \tau}$, and summarise the distribution of the prediction errors $Y_{i \tau}-\hat{Y}_{i \tau}, \tau=2005 . q 1, \ldots 2011 . q 4$, by means of the mean error ( $\mathrm{ME}_{i}$, the average of the 28 prediction errors), the prediction error variance, $V_{i}$, the mean square forecast error,

$$
\operatorname{MSFE}_{i}=\frac{1}{M} \sum_{\tau=1}^{M}\left(Y_{i \tau}-\hat{Y}_{i \tau}\right)^{2}=\mathrm{ME}_{i}^{2}+V_{i}
$$


Table 2: Variance decomposition for each of the monthly indicators. The table reports the median value of the percentage share of the variance explained by each factor, along with the 33th and 66th percentiles of the sampling distribution of the statistic. For variable names and definitions, see Tables 4 and 5 .

\begin{tabular}{|c|c|c|c|c|c|c|c|c|c|c|c|c|}
\hline \multirow[b]{3}{*}{ Sector A- } & \multicolumn{3}{|c|}{ Common European factor } & \multicolumn{3}{|c|}{ Country-specific factors } & \multicolumn{3}{|c|}{ Component-specific factors } & \multicolumn{3}{|c|}{ Idiosyncratic } \\
\hline & $1 / 3$ & Med & $2 / 3$ & $1 / 3$ & Med & $2 / 3$ & $1 / 3$ & Med & $2 / 3$ & $1 / 3$ & Med & $2 / 3$ \\
\hline & 26.69 & 47.66 & 67.31 & 2.36 & 4.22 & 5.97 & 2.05 & 3.66 & 5.17 & 24.89 & 44.44 & 62.77 \\
\hline Germany & 11.68 & 20.86 & 29.46 & 0.49 & 0.89 & 1.25 & 2.16 & 3.85 & 5.45 & 41.66 & 74.38 & 105.06 \\
\hline France & 1.16 & 2.07 & 2.92 & 0.37 & 0.67 & 0.94 & 6.56 & 11.71 & 16.54 & 47.90 & 85.53 & 120.82 \\
\hline Spain & 21.22 & 37.89 & 53.52 & 1.32 & 2.36 & 3.33 & 5.24 & 9.36 & 13.22 & 28.21 & 50.37 & 71.15 \\
\hline Italy & 27.14 & 48.47 & 68.46 & 0.20 & 0.35 & 0.50 & 0.11 & 0.20 & 0.29 & 28.54 & 50.96 & 71.97 \\
\hline \multicolumn{13}{|c|}{ Sector C-D-E } \\
\hline EA & 32.27 & 57.62 & 81.38 & 8.51 & 15.20 & 21.47 & 0.99 & 1.78 & 2.51 & 14.21 & 25.38 & 35.86 \\
\hline Germany & 29.76 & 53.14 & 75.06 & 14.29 & 25.52 & 36.04 & 0.59 & 1.05 & 1.49 & 11.35 & 20.27 & 28.63 \\
\hline France & 31.22 & 55.75 & 78.74 & 0.56 & 1.01 & 1.42 & 0.36 & 0.65 & 0.92 & 23.85 & 42.58 & 60.15 \\
\hline Spain & 31.02 & 55.38 & 78.22 & 5.33 & 9.53 & 13.46 & 3.74 & 6.69 & 9.45 & 15.90 & 28.39 & 40.10 \\
\hline Italy & 17.90 & 31.96 & 45.14 & 9.27 & 16.56 & 23.39 & 8.13 & 14.51 & 20.5 & 20.69 & 36.95 & 52.19 \\
\hline \multicolumn{13}{|l|}{ Sector F } \\
\hline EA & 5.33 & 9.53 & 13.46 & 4.29 & 7.66 & 10.82 & 10.68 & 19.07 & 26.94 & 35.69 & 63.73 & 90.01 \\
\hline Germany & 0.2 & 0.36 & 0.52 & 3.45 & 6.16 & 8.70 & 11.36 & 20.29 & 28.67 & 40.98 & 73.16 & 103.34 \\
\hline France & 8.56 & 15.30 & 21.61 & 7.97 & 14.23 & 20.11 & 0.28 & 0.50 & 0.71 & 39.18 & 69.95 & 98.8 \\
\hline Spain & 0.49 & 0.89 & 1.25 & 14.01 & 25.02 & 35.34 & 1.72 & 3.08 & 4.35 & 39.76 & 71.00 & 100.28 \\
\hline Italy & 4.15 & 7.42 & 10.49 & 6.84 & 12.22 & 17.27 & 2.50 & 4.47 & 6.31 & 42.49 & 75.87 & 107.16 \\
\hline \multicolumn{13}{|c|}{ Sector G-H-I } \\
\hline EA & 31.17 & 55.66 & 78.62 & 6.17 & 11.02 & 15.56 & 0.86 & 1.55 & 2.19 & 17.78 & 31.75 & 44.85 \\
\hline Germany & 25.88 & 46.21 & 65.27 & 5.12 & 9.15 & 12.93 & 1.50 & 2.68 & 3.79 & 23.49 & 41.94 & 59.24 \\
\hline France & 22.35 & 39.9 & 56.36 & 1.23 & 2.20 & 3.11 & 0.91 & 1.64 & 2.31 & 31.50 & 56.24 & 79.44 \\
\hline Spain & 29.14 & 52.04 & 73.50 & 8.68 & 15.51 & 21.91 & 0.27 & 0.48 & 0.68 & 17.90 & 31.96 & 45.14 \\
\hline Italy & 26.64 & 47.56 & 67.18 & 4.59 & 8.19 & 11.57 & 0.51 & 0.92 & 1.30 & 24.25 & 43.31 & 61.17 \\
\hline \multicolumn{13}{|c|}{ Sector J-K } \\
\hline EA & 38.90 & 69.46 & 98.11 & 4.32 & 7.71 & 10.90 & 0.01 & 0.03 & 0.05 & 12.76 & 22.78 & 32.18 \\
\hline Germany & 38.54 & 68.81 & 97.20 & 0.54 & 0.98 & 1.38 & 0.37 & 0.66 & 0.93 & 16.54 & 29.53 & 41.71 \\
\hline France & 12.90 & 23.04 & 32.54 & 6.38 & 11.40 & 16.10 & 1.64 & 2.92 & 4.13 & 35.07 & 62.62 & 88.46 \\
\hline Spain & 26.00 & 46.43 & 65.58 & 11.32 & 20.21 & 28.55 & 0.12 & 0.22 & 0.31 & 18.55 & 33.12 & 46.79 \\
\hline Italy & 30.71 & 54.84 & 77.46 & 3.76 & 6.71 & 9.48 & 0.13 & 0.23 & 0.33 & 21.39 & 38.20 & 53.95 \\
\hline Sector L- & & & & & & & & & & & & \\
\hline EA & 4.39 & 7.84 & 11.08 & 9.39 & 16.78 & 23.70 & 0.11 & 0.19 & 0.28 & 42.10 & 75.17 & 106.17 \\
\hline Germany & 7.91 & 14.13 & 19.95 & 4.53 & 8.09 & 11.43 & 0.04 & 0.07 & 0.10 & 43.51 & 77.69 & 109.74 \\
\hline France & 6.47 & 11.56 & 16.33 & 0.18 & 0.33 & 0.46 & 1.30 & 2.33 & 3.29 & 48.03 & 85.76 & 121.14 \\
\hline Spain & 29.81 & 53.23 & 75.19 & 2.61 & 4.66 & 6.58 & 0.20 & 0.37 & 0.52 & 23.37 & 41.72 & 58.93 \\
\hline Italy & 11.72 & 20.94 & 29.57 & 0.38 & 0.69 & 0.97 & 4.62 & 8.24 & 11.65 & 39.27 & 70.11 & 99.04 \\
\hline Sector Tl & & & & & & & & & & & & \\
\hline EA & 18.08 & 32.28 & 45.59 & 4.04 & 7.22 & 10.20 & 15.68 & 28.00 & 39.56 & 18.19 & 32.48 & 45.87 \\
\hline Germany & 3.07 & 5.49 & 7.75 & 3.87 & 6.91 & 9.76 & 18.80 & 33.57 & 47.42 & 30.25 & 54.01 & 76.29 \\
\hline France & 6.43 & 11.49 & 16.23 & 7.11 & 12.70 & 17.94 & 8.18 & 14.61 & 20.63 & 34.27 & 61.19 & 86.43 \\
\hline Spain & 18.73 & 33.45 & 47.25 & 9.99 & 17.83 & 25.19 & 2.94 & 5.25 & 7.42 & 24.33 & 43.45 & 61.37 \\
\hline Italy & 21.00 & 37.50 & 52.96 & 2.88 & 5.14 & 7.27 & 11.68 & 20.86 & 29.46 & 20.43 & 36.49 & 51.54 \\
\hline Sector FC & & & & & & & & & & & & \\
\hline EA & 3.53 & 6.30 & 8.90 & 9.18 & 16.4 & 23.16 & 0.41 & 0.73 & 1.04 & 42.87 & 76.55 & 108.12 \\
\hline Germany & 0.04 & 0.08 & 0.11 & 0.00 & 0.00 & 0.00 & 7.16 & 12.79 & 18.07 & 48.79 & 87.11 & 123.05 \\
\hline France & 2.53 & 4.53 & 6.40 & 7.71 & 13.77 & 19.45 & 0.10 & 0.18 & 0.25 & 45.65 & 81.51 & 115.13 \\
\hline Spain & 17.20 & 30.71 & 43.38 & 0.39 & 0.70 & 0.99 & 0.14 & 0.25 & 0.36 & 38.26 & 68.32 & 96.50 \\
\hline Italy & 14.96 & 26.71 & 37.72 & 3.86 & 6.89 & 9.73 & 0.00 & 0.00 & 0.00 & 37.18 & 66.39 & 93.77 \\
\hline Sector GC & & & & & & & & & & & & \\
\hline EA & 31.85 & 56.86 & 80.32 & 5.88 & 10.49 & 14.82 & 0.50 & 0.90 & 1.28 & 17.76 & 31.72 & 44.80 \\
\hline Germany & 9.58 & 17.10 & 24.16 & 0.01 & 0.03 & 0.04 & 1.59 & 2.85 & 4.02 & 44.80 & 80.00 & 113.00 \\
\hline France & 8.55 & 15.27 & 21.56 & 2.85 & 5.10 & 7.20 & 0.08 & 0.15 & 0.21 & 44.51 & 79.47 & 112.25 \\
\hline Spain & 29.38 & 52.45 & 74.09 & 9.64 & 17.22 & 24.32 & 0.52 & 0.93 & 1.31 & 16.45 & 29.38 & 41.50 \\
\hline Italy & 35.43 & 63.26 & 89.35 & 3.17 & 5.66 & 7.99 & 0.44 & 0.79 & 1.12 & 16.95 & 30.27 & 42.76 \\
\hline Sector EX & & & & & & & & & & & & \\
\hline EA & 0.88 & 1.57 & 2.22 & 2.90 & 5.18 & 7.31 & 14.31 & 25.56 & 36.10 & 37.90 & 67.68 & 95.59 \\
\hline Germany & 0.29 & 0.52 & 0.74 & 0.12 & 0.22 & 0.31 & 14.35 & 25.63 & 36.20 & 41.23 & 73.61 & 103.97 \\
\hline France & 4.37 & 7.81 & 11.04 & 0.33 & 0.59 & 0.83 & 3.89 & 6.95 & 9.82 & 47.40 & 84.63 & 119.54 \\
\hline Spain & 0.00 & 0.00 & 0.00 & 1.61 & 2.88 & 4.07 & 2.00 & 3.58 & 5.06 & 52.38 & 93.52 & 132.10 \\
\hline Italy & 2.75 & 4.92 & 6.95 & 1.55 & 2.77 & 3.92 & 16.13 & 28.80 & 40.68 & 35.56 & 63.50 & 89.69 \\
\hline Sector IM & & & & & & & & & & & & \\
\hline EA & 0.49 & 0.89 & 1.25 & 12.44 & 22.22 & 31.39 & 6.98 & 12.47 & 17.62 & 36.07 & 64.40 & 90.97 \\
\hline Germany & 3.22 & 5.76 & 8.14 & 13.71 & 24.48 & 34.58 & 3.67 & 6.55 & 9.25 & 35.39 & 63.19 & 89.26 \\
\hline France & 19.31 & 34.47 & 48.70 & 2.48 & 4.43 & 6.25 & 3.07 & 5.48 & 7.74 & 31.14 & 55.60 & 78.54 \\
\hline Spain & 25.01 & 44.66 & 63.09 & 8.09 & 14.46 & 20.42 & 0.13 & 0.23 & 0.33 & 22.75 & 40.63 & 57.39 \\
\hline Italy & 4.99 & 8.91 & 12.59 & 3.18 & 5.69 & 8.03 & 0.32 & 0.57 & 0.81 & 47.50 & 84.82 & 119.80 \\
\hline
\end{tabular}


Table 2: Continued: Variance decomposition for each of the monthly indicators. The table reports the median value of the percentage share of the variance explained by each factor, along with the 33th and 66th percentiles of the sampling distribution of the statistic. For variable names and definitions, see Tables 4 and 5.

\begin{tabular}{|c|c|c|c|c|c|c|c|c|c|c|c|c|}
\hline \multirow{3}{*}{ Series: sts_inpr_msa } & \multicolumn{3}{|c|}{ Common European factor } & \multicolumn{3}{|c|}{ Country-specific factors } & \multicolumn{3}{|c|}{ Component-specific factors } & \multicolumn{3}{|c|}{ Idiosyncratic } \\
\hline & $1 / 3$ & Med & $2 / 3$ & $1 / 3$ & Med & $2 / 3$ & $1 / 3$ & Med & $2 / 3$ & $1 / 3$ & Med & $2 / 3$ \\
\hline & 1781 & 3181 & 4403 & 1264 & 2257 & 3188 & 2552 & 4557 & 6436 & 000 & 004 & 006 \\
\hline Germany & 16.07 & 28.69 & 40.53 & $\begin{array}{l}12.04 \\
17.48\end{array}$ & 31.21 & $\begin{array}{r}31.88 \\
44.09\end{array}$ & $\begin{array}{l}25.52 \\
22.39\end{array}$ & $\begin{array}{l}45.57 \\
39.98\end{array}$ & $\begin{array}{l}04.50 \\
56.47\end{array}$ & $\begin{array}{l}0.02 \\
0.05\end{array}$ & $\begin{array}{l}0.04 \\
0.10\end{array}$ & $\begin{array}{l}0.00 \\
0.14\end{array}$ \\
\hline Spain & 0.00 & 0.00 & 0.00 & 5.91 & 10.56 & 14.92 & 2.36 & 4.22 & 5.96 & 47.72 & 85.20 & 120.34 \\
\hline France & 3.79 & 6.76 & 9.56 & 13.52 & 24.14 & 34.10 & 16.22 & 28.96 & 40.91 & 22.46 & 40.11 & 56.66 \\
\hline Italy & 2.44 & 4.35 & 6.15 & 6.47 & 11.56 & 16.33 & 25.76 & 45.99 & 64.96 & 21.32 & 38.08 & 53.78 \\
\hline Series: st & & & & & & & & & & & & \\
\hline EA & 8.19 & 14.62 & 20.66 & 0.28 & 0.51 & 0.72 & 47.42 & 84.68 & 119.61 & 0.09 & 0.17 & 0.25 \\
\hline Germany & 5.76 & 10.29 & 14.54 & 0.42 & 0.75 & 1.06 & 27.37 & 48.87 & 69.03 & 22.44 & 40.07 & 56.60 \\
\hline Spain & 0.00 & 0.00 & 0.00 & 1.23 & 2.20 & 3.10 & 9.02 & 16.12 & 22.76 & 45.74 & 81.67 & 115.35 \\
\hline France & 2.85 & 5.10 & 7.20 & 0.87 & 1.55 & 2.19 & 19.75 & 35.27 & 49.81 & 32.52 & 58.07 & 82.02 \\
\hline Italy & 3.55 & 6.34 & 8.96 & 0.01 & 0.02 & 0.03 & 16.87 & 30.13 & 42.56 & 35.56 & 63.49 & 89.68 \\
\hline Series: $\mathrm{s}$ & & & & & & & & & & & & \\
\hline EA & 22.31 & 39.83 & 56.26 & 25.74 & 45.96 & 64.92 & 3.58 & 6.39 & 9.03 & 4.37 & 7.80 & 11.02 \\
\hline Germany & 18.25 & 32.58 & 46.02 & 35.74 & 63.81 & 90.13 & 1.27 & 2.27 & 3.21 & 0.73 & 1.32 & 1.86 \\
\hline Spain & 18.98 & 33.90 & 47.88 & 11.79 & 21.06 & 29.75 & 3.67 & 6.55 & 9.25 & 21.55 & 38.47 & 54.35 \\
\hline France & 1.29 & 2.30 & 3.25 & 46.03 & 82.19 & 116.10 & 1.47 & 2.62 & 3.71 & 7.20 & 12.86 & 18.17 \\
\hline Italy & 7.84 & 13.99 & 19.77 & 33.38 & 59.6 & 84.18 & 2.56 & 4.58 & 6.47 & 12.22 & 21.81 & 30.81 \\
\hline Series: Is & & & & & & & & & & & & \\
\hline EA & 15.25 & 27.23 & 38.47 & 4.63 & 8.26 & 11.67 & 8.73 & 15.58 & 22.01 & 27.39 & 48.90 & 69.07 \\
\hline Germany & 13.98 & 24.96 & 35.26 & 5.18 & 9.25 & 13.07 & 8.62 & 15.39 & 21.74 & 28.21 & 50.38 & 71.16 \\
\hline Spain & 0.87 & 1.56 & 2.2 & 5.8 & 10.35 & 14.63 & 10.35 & 18.49 & 26.11 & 38.97 & 69.58 & 98.28 \\
\hline France & 1.77 & 3.17 & 4.48 & 0.21 & 0.38 & 0.53 & 0.34 & 0.60 & 0.85 & 53.67 & 95.83 & 135.36 \\
\hline Italy & 2.76 & 4.92 & 6.96 & 5.19 & 9.26 & 13.09 & 4.42 & 7.90 & 11.15 & 43.63 & 77.90 & 110.03 \\
\hline Series: $b$ & & & & & & & & & & & & \\
\hline EA & 23.40 & 41.79 & 59.02 & 0.05 & 0.09 & 0.13 & 31.99 & 57.12 & 80.69 & 0.55 & 0.98 & 1.39 \\
\hline Germany & 22.48 & 40.14 & 56.69 & 0.00 & 0.00 & 0.00 & 31.37 & 56.01 & 79.11 & 2.15 & 3.84 & 5.43 \\
\hline Spain & 22.89 & 40.87 & 57.73 & 0.08 & 0.15 & 0.22 & 31.08 & 55.49 & 78.38 & 1.94 & 3.46 & 4.89 \\
\hline France & 20.14 & 35.97 & 50.81 & 0.08 & 0.15 & 0.21 & 34.55 & 61.69 & 87.13 & 1.22 & 2.18 & 3.08 \\
\hline Italy & 23.88 & 42.63 & 60.22 & 0.01 & 0.02 & 0.03 & 31.47 & 56.20 & 79.38 & 0.63 & 1.13 & 1.60 \\
\hline Series: $\mathbf{b}$ & & & & & & & & & & & & \\
\hline EA & 55.42 & 98.95 & 139.76 & 0.01 & 0.03 & 0.04 & 0.45 & 0.81 & 1.15 & 0.11 & 0.19 & 0.28 \\
\hline Germany & 51.57 & 92.09 & 130.07 & 0.00 & 0.00 & 0.01 & 0.53 & 0.95 & 1.35 & 3.88 & 6.93 & 9.80 \\
\hline Spain & 53.84 & 96.13 & 135.79 & 0.02 & 0.04 & 0.05 & 0.57 & 1.03 & 1.46 & 1.56 & 2.78 & 3.93 \\
\hline France & 54.52 & 97.35 & 137.50 & 0.00 & 0.00 & 0.00 & 0.43 & 0.77 & 1.09 & 1.04 & 1.87 & 2.64 \\
\hline Italy & 55.33 & 98.80 & 139.55 & 0.01 & 0.03 & 0.04 & 0.45 & 0.81 & 1.14 & 0.19 & 0.35 & 0.50 \\
\hline Series: $\mathrm{s}$ & & & & & & & & & & & & \\
\hline Germany & 1.44 & 2.58 & 3.64 & 0.00 & 0.00 & 0.01 & 0.30 & 0.55 & 0.78 & 54.24 & 96.85 & 136.80 \\
\hline Spain & 0.00 & 0.00 & 0.00 & 0.49 & 0.88 & 1.24 & 0.62 & 1.12 & 1.58 & 54.88 & 97.99 & 138.41 \\
\hline Series: $\mathrm{s}$ & & & & & & & & & & & & \\
\hline $\begin{array}{l}\text { Germany } \\
\text { Series: }\end{array}$ & 0.05 & 0.09 & 0.13 & 0.00 & 0.00 & 0.00 & 0.00 & 0.00 & 0.00 & 55.95 & 99.90 & 141.11 \\
\hline Germany & 0.10 & 0.18 & 0.26 & 0.03 & 0.05 & 0.08 & 0.77 & 1.37 & 1.94 & 55.09 & 98.37 & 138.94 \\
\hline Series: $\mathrm{s}$ & & & & & & & & & & & & \\
\hline EA & 6.72 & 12.00 & 16.95 & 3.26 & 5.83 & 8.23 & 28.04 & 50.07 & 70.72 & 17.97 & 32.09 & 45.33 \\
\hline Germany & 3.59 & 6.42 & 9.06 & 3.00 & 5.36 & 7.57 & 20.54 & 36.68 & 51.81 & 28.85 & 51.52 & 72.77 \\
\hline Spain & 0.73 & 1.31 & 1.86 & 4.69 & 8.37 & 11.83 & 0.87 & 1.55 & 2.20 & 49.70 & 88.74 & 125.35 \\
\hline France & 3.52 & 6.28 & 8.87 & 1.84 & 3.29 & 4.66 & 15.05 & 26.87 & 37.96 & 35.58 & 63.53 & 89.74 \\
\hline Series: $\mathrm{e}$ & & & & & & & & & & & & \\
\hline Germany & 3.89 & 6.94 & 9.81 & 0.49 & 0.87 & 1.23 & 1.54 & 2.75 & 3.88 & 50.08 & 89.42 & 126.30 \\
\hline Spain & 0.16 & 0.29 & 0.42 & 3.75 & 6.70 & 9.47 & 2.65 & 4.73 & 6.68 & 49.43 & 88.25 & 124.66 \\
\hline France & 1.69 & 3.01 & 4.26 & 0.78 & 1.40 & 1.98 & 3.96 & 7.07 & 9.99 & 49.56 & 88.50 & 125.00 \\
\hline Italy & 16.91 & 30.20 & 42.66 & 3.82 & 6.82 & 9.64 & 1.50 & 2.69 & 3.80 & 33.75 & 60.27 & 85.13 \\
\hline Series: in & & & & & & & & & & & & \\
\hline Germany & 4.34 & 7.75 & 10.95 & 0.38 & 0.69 & 0.98 & 0.00 & 0.00 & 0.00 & 51.27 & 91.54 & 129.30 \\
\hline Spain & 0.15 & 0.27 & 0.38 & 0.00 & 0.00 & 0.00 & 0.53 & 0.95 & 1.35 & 55.32 & 98.77 & 139.51 \\
\hline France & 9.92 & 17.72 & 25.03 & 0.82 & 1.46 & 2.07 & 0.49 & 0.87 & 1.24 & 44.76 & 79.92 & 112.89 \\
\hline Italy & 0.14 & 0.26 & 0.37 & 4.95 & 8.84 & 12.49 & 1.49 & 2.66 & 3.77 & 49.41 & 88.22 & 124.6 \\
\hline Series: $\mathrm{n}$ & & & & & & & & & & & & \\
\hline EA & 27.25 & 48.66 & 68.73 & 0.05 & 0.10 & 0.14 & 28.45 & 50.80 & 71.75 & 0.24 & 0.43 & 0.61 \\
\hline Germany & 20.23 & 36.13 & 51.03 & 0.01 & 0.03 & 0.04 & 31.86 & 56.89 & 80.36 & 3.88 & 6.94 & 9.80 \\
\hline Spain & 28.14 & 50.25 & 70.98 & 0.00 & 0.01 & 0.02 & 21.38 & 38.18 & 53.93 & 6.46 & 11.54 & 16.30 \\
\hline France & 27.95 & 49.91 & 70.50 & 0.00 & 0.00 & 0.00 & 27.02 & 48.25 & 68.15 & 1.02 & 1.82 & 2.57 \\
\hline Italy & 27.35 & 48.83 & 68.97 & 0.01 & 0.02 & 0.03 & 25.08 & 44.77 & 63.24 & 3.56 & 6.36 & 8.98 \\
\hline Series: $\mathrm{n}$ & & & & & & & & & & & & \\
\hline EA & 24.59 & 43.90 & 62.01 & 5.24 & 9.36 & 13.22 & 21.80 & 38.92 & 54.98 & 4.36 & 7.79 & 11.01 \\
\hline Germany & 18.36 & 32.78 & 46.31 & 0.00 & 0.00 & 0.00 & 19.30 & 34.47 & 48.68 & 18.33 & 32.73 & 46.23 \\
\hline Spain & 17.03 & 30.41 & 42.95 & 2.50 & 4.46 & 6.31 & 16.66 & 29.75 & 42.02 & 19.80 & 35.36 & 49.95 \\
\hline France & 27.98 & 49.96 & 70.57 & 0.59 & 1.06 & 1.50 & 15.73 & 28.09 & 39.67 & 11.69 & 20.87 & 29.49 \\
\hline Italy & 24.21 & 43.24 & 61.07 & 0.94 & 1.68 & 2.38 & 15.34 & 27.40 & 38.70 & 15.49 & 27.66 & 39.07 \\
\hline Series: $\mathrm{n}$ & & & & & & & & & & & & \\
\hline EA & 1.66 & 2.97 & 4.20 & 0.18 & 0.32 & 0.45 & 0.06 & 0.12 & 0.17 & 54.08 & 96.57 & 136.40 \\
\hline Germany & 1.29 & 2.30 & 3.25 & 0.22 & 0.39 & 0.56 & 0.29 & 0.53 & 0.75 & 54.19 & 96.76 & 136.67 \\
\hline Spain & 0.04 & 0.08 & 0.11 & 0.70 & 1.25 & 1.76 & 0.45 & 0.81 & 1.15 & 54.8 & 97.84 & 138.20 \\
\hline France & 0.69 & 1.23 & 1.74 & 0.11 & 0.20 & 0.28 & 0.01 & 0.02 & 0.02 & 55.18 & 98.53 & 139.17 \\
\hline Italy & 4.07 & 7.28 & 10.28 & 0.02 & 0.04 & 0.05 & 0.05 & 0.10 & 0.14 & 51.84 & 92.57 & 130.75 \\
\hline
\end{tabular}


Table 2: Continued: Variance decomposition for each of the monthly indicators. The table reports the median value of the percentage share of the variance explained by each factor, along with the 33th and 66th percentiles of the sampling distribution of the statistic. For variable names and definitions, see Tables 4 and 5.

\begin{tabular}{|c|c|c|c|c|c|c|c|c|c|c|c|c|}
\hline \multirow[b]{3}{*}{ Series: bssi_GCF } & \multicolumn{3}{|c|}{ Common European factor } & \multicolumn{3}{|c|}{ Country-specific factors } & \multicolumn{3}{|c|}{ Component-specific factors } & \multicolumn{3}{|c|}{ Idiosyncratic } \\
\hline & $1 / 3$ & Med & $2 / 3$ & $1 / 3$ & Med & $2 / 3$ & $1 / 3$ & Med & $2 / 3$ & $1 / 3$ & Med & $2 / 3$ \\
\hline & 2764 & 4935 & 6971 & 019 & 0.34 & 0.48 & 0.00 & 000 & 0.00 & 28.16 & 5029 & 7103 \\
\hline Germany & 24.12 & 43.06 & 60.83 & 0.07 & 0.13 & 0.18 & 0.02 & 0.04 & 0.06 & 31.78 & 56.75 & 80.15 \\
\hline Spain & 25.76 & 46.00 & 64.97 & 0.77 & 1.39 & 1.96 & 0.18 & 0.33 & 0.47 & 29.27 & 52.26 & 73.82 \\
\hline France & 12.07 & 21.56 & 30.46 & 0.73 & 1.31 & 1.86 & 0.28 & 0.51 & 0.73 & 42.90 & 76.59 & 108.18 \\
\hline Italy & 22.65 & 40.44 & 57.13 & 0.17 & 0.30 & 0.43 & 0.17 & 0.31 & 0.44 & 33.00 & 58.92 & 83.22 \\
\hline \multicolumn{13}{|c|}{ Series: sts_intvd_m } \\
\hline EA & 11.00 & 19.64 & 27.75 & 25.21 & 45.02 & 63.58 & 0.12 & 0.22 & 0.32 & 19.66 & 35.10 & 49.58 \\
\hline Germany & 15.15 & 27.06 & 38.23 & 33.10 & 59.10 & 83.48 & 0.30 & 0.54 & 0.77 & 7.43 & 13.27 & 18.75 \\
\hline Spain & 8.52 & 15.21 & 21.48 & 8.70 & 15.53 & 21.94 & 1.02 & 1.83 & 2.58 & 37.75 & 67.41 & 95.22 \\
\hline France & 0.83 & 1.49 & 2.10 & 42.59 & 76.04 & 107.41 & 0.28 & 0.51 & 0.72 & 12.29 & 21.94 & 30.99 \\
\hline Italy & 0.00 & 0.00 & 0.00 & 26.46 & 47.26 & 66.75 & 0.01 & 0.02 & 0.02 & 29.52 & 52.71 & 74.45 \\
\hline \multicolumn{13}{|c|}{ Series: sts_rt_careg } \\
\hline EA & 0.59 & 1.05 & 1.48 & 2.28 & 4.08 & 5.76 & 51.06 & 91.17 & 128.77 & 2.06 & 3.69 & 5.21 \\
\hline Germany & 0.92 & 1.65 & 2.34 & 0.22 & 0.40 & 0.56 & 13.35 & 23.84 & 33.68 & 41.49 & 74.09 & 104.65 \\
\hline Spain & 0.18 & 0.33 & 0.47 & 2.52 & 4.50 & 6.36 & 9.24 & 16.50 & 23.31 & 44.05 & 78.65 & 111.09 \\
\hline France & 1.61 & 2.87 & 4.06 & 0.07 & 0.13 & 0.18 & 12.94 & 23.11 & 32.64 & 41.37 & 73.88 & 104.35 \\
\hline Italy & 7.31 & 13.05 & 18.43 & 0.74 & 1.32 & 1.87 & 13.13 & 23.44 & 33.11 & 34.82 & 62.17 & 87.81 \\
\hline \multicolumn{13}{|c|}{ Series: bssi_FCE } \\
\hline EA & 15.38 & 27.47 & 38.80 & 1.07 & 1.91 & 2.70 & 1.76 & 3.14 & 4.44 & 37.78 & 67.46 & 95.29 \\
\hline Germany & 8.64 & 15.42 & 21.79 & 0.10 & 0.19 & 0.27 & 0.22 & 0.40 & 0.56 & 47.03 & 83.97 & 118.61 \\
\hline Spain & 6.90 & 12.33 & 17.42 & 0.08 & 0.14 & 0.20 & 4.32 & 7.71 & 10.90 & 44.69 & 79.80 & 112.71 \\
\hline France & 3.24 & 5.79 & 8.18 & 0.46 & 0.82 & 1.17 & 0.55 & 0.99 & 1.40 & 51.74 & 92.38 & 130.48 \\
\hline Italy & 8.78 & 15.68 & 22.15 & 0.26 & 0.46 & 0.66 & 0.24 & 0.44 & 0.62 & 46.71 & 83.40 & 117.80 \\
\hline \multicolumn{13}{|c|}{ Series: bsco_FCE } \\
\hline $\mathrm{EA}$ & 7.47 & 13.33 & 18.84 & 1.67 & 2.99 & 4.22 & 45.49 & 81.23 & 114.74 & 1.36 & 2.43 & 3.43 \\
\hline Germany & 2.26 & 4.03 & 5.70 & 1.32 & 2.37 & 3.35 & 21.00 & 37.49 & 52.96 & 31.41 & 56.09 & 79.22 \\
\hline Spain & 5.99 & 10.70 & 15.11 & 0.42 & 0.76 & 1.08 & 11.07 & 19.77 & 27.92 & 38.51 & 68.75 & 97.11 \\
\hline France & 2.03 & 3.63 & 5.13 & 0.00 & 0.00 & 0.01 & 11.97 & 21.38 & 30.20 & 41.98 & 74.97 & 105.89 \\
\hline Italy & 0.12 & 0.22 & 0.31 & 0.66 & 1.18 & 1.67 & 8.66 & 15.47 & 21.85 & 46.55 & 83.11 & 117.40 \\
\hline Series: sts & & & & & & & & & & & & \\
\hline EA & 9.59 & 17.13 & 24.20 & 2.30 & 4.10 & 5.80 & 39.79 & 71.04 & 100.35 & 4.31 & 7.70 & 10.88 \\
\hline Germany & 1.47 & 2.62 & 3.70 & 0.35 & 0.63 & 0.89 & 24.02 & 42.88 & 60.57 & 30.16 & 53.85 & 76.06 \\
\hline Spain & 14.84 & 26.50 & 37.43 & 4.62 & 8.25 & 11.66 & 3.77 & 6.73 & 9.51 & 32.76 & 58.50 & 82.63 \\
\hline France & 4.89 & 8.74 & 12.35 & 4.12 & 7.36 & 10.39 & 15.93 & 28.44 & 40.18 & 31.05 & 55.44 & 78.31 \\
\hline Italy & 5.30 & 9.47 & 13.37 & 0.00 & 0.01 & 0.02 & 18.95 & 33.84 & 47.81 & 31.73 & 56.66 & 80.03 \\
\hline Series: sts & & & & & & & & & & & & \\
\hline Germany & 0.24 & 0.43 & 0.61 & 4.10 & 7.32 & 10.34 & 0.51 & 0.91 & 1.29 & 51.14 & 91.32 & 128.98 \\
\hline $\begin{array}{l}\text { Series: sb } \\
\text { EA }\end{array}$ & 0.92 & 1.64 & 2.32 & 1.57 & 2.81 & 3.97 & 4.51 & 8.06 & 11.39 & 48.99 & 87.47 & 123.54 \\
\hline Germany & 1.60 & 2.87 & 4.05 & 0.19 & 0.35 & 0.49 & 4.41 & 7.87 & 11.12 & 49.78 & 88.89 & 125.56 \\
\hline Spain & 1.63 & 2.91 & 4.11 & 0.03 & 0.05 & 0.08 & 1.41 & 2.53 & 3.57 & 52.92 & 94.49 & 133.47 \\
\hline France & 0.95 & 1.69 & 2.39 & 2.14 & 3.82 & 5.40 & 0.89 & 1.60 & 2.26 & 52.01 & 92.86 & 131.17 \\
\hline Italy & 1.03 & 1.84 & 2.60 & 0.06 & 0.12 & 0.17 & 0.54 & 0.96 & 1.36 & 54.36 & 97.06 & 137.10 \\
\hline Series: ap & & & & & & & & & & & & \\
\hline EA & 4.15 & 7.41 & 10.47 & 0.00 & 0.00 & 0.00 & 4.06 & 7.26 & 10.25 & 47.78 & 85.32 & 120.51 \\
\hline Germany & 9.94 & 17.75 & 25.07 & 0.07 & 0.12 & 0.18 & 0.86 & 1.54 & 2.18 & 45.12 & 80.57 & 113.80 \\
\hline Spain & 0.00 & 0.01 & 0.01 & 0.34 & 0.61 & 0.86 & 2.71 & 4.84 & 6.84 & 52.94 & 94.53 & 133.52 \\
\hline France & 1.27 & 2.27 & 3.20 & 0.20 & 0.36 & 0.51 & 2.36 & 4.22 & 5.96 & 52.16 & 93.14 & 131.56 \\
\hline Italy & 3.03 & 5.41 & 7.64 & 0.08 & 0.15 & 0.21 & 0.93 & 1.67 & 2.35 & 51.95 & 92.76 & 131.02 \\
\hline Series: ap & & & & & & & & & & & & \\
\hline Germany & 1.65 & 2.94 & 4.16 & 0.03 & 0.05 & 0.07 & 32.64 & 58.27 & 82.31 & 21.68 & 38.71 & 54.68 \\
\hline Spain & 0.69 & 1.24 & 1.75 & 0.20 & 0.37 & 0.52 & 19.41 & 34.66 & 48.95 & 35.69 & 63.72 & 90.00 \\
\hline France & 0.07 & 0.13 & 0.19 & 0.06 & 0.11 & 0.16 & 40.21 & 71.79 & 101.40 & 15.65 & 27.95 & 39.48 \\
\hline Italy & 0.58 & 1.04 & 1.47 & 0.04 & 0.07 & 0.10 & 15.49 & 27.67 & 39.08 & 39.88 & 71.20 & 100.58 \\
\hline Series: ap & & & & & & & & & & & & \\
\hline EA & 0.00 & 0.00 & 0.00 & 0.00 & 0.00 & 0.00 & 51.94 & 92.73 & 130.98 & 4.06 & 7.25 & 10.25 \\
\hline Germany & 0.41 & 0.73 & 1.03 & 0.05 & 0.10 & 0.14 & 42.30 & 75.53 & 106.69 & 13.23 & 23.62 & 33.37 \\
\hline Spain & 0.98 & 1.74 & 2.47 & 0.01 & 0.03 & 0.04 & 25.88 & 46.22 & 65.28 & 29.12 & 51.99 & 73.44 \\
\hline France & 0.02 & 0.05 & 0.07 & 0.13 & 0.23 & 0.33 & 0.15 & 0.26 & 0.38 & 55.69 & 99.44 & 140.45 \\
\hline Italy & 0.74 & 1.33 & 1.88 & 0.13 & 0.24 & 0.34 & 0.38 & 0.68 & 0.96 & 54.74 & 97.73 & 138.05 \\
\hline Series: Im & & & & & & & & & & & & \\
\hline EA & 1.68 & 3.00 & 4.24 & 1.62 & 2.89 & 4.08 & 0.39 & 0.70 & 0.99 & 52.30 & 93.39 & 131.91 \\
\hline Germany & 0.05 & 0.10 & 0.14 & 0.00 & 0.01 & 0.02 & 0.00 & 0.00 & 0.00 & 55.93 & 99.87 & 141.06 \\
\hline Spain & 0.00 & 0.00 & 0.00 & 0.03 & 0.07 & 0.10 & 0.05 & 0.10 & 0.14 & 55.91 & 99.82 & 140.99 \\
\hline France & 0.71 & 1.28 & 1.81 & 0.09 & 0.16 & 0.23 & 0.04 & 0.08 & 0.12 & 55.14 & 98.45 & 139.06 \\
\hline Italy & 2.92 & 5.21 & 7.36 & 5.44 & 9.72 & 13.73 & 0.04 & 0.08 & 0.12 & 47.59 & 84.96 & 120.01 \\
\hline
\end{tabular}


the mean absolute error (the average of $\left|Y_{i \tau}-\hat{Y}_{i \tau}\right|$ ), and the mean absolute percentage error,

$$
\operatorname{MAPE}_{i}=\frac{1}{M} \sum_{\tau=1}^{M} \frac{\left|Y_{i \tau}-\hat{Y}_{i \tau}\right|}{Y_{i \tau}}
$$

The values of the above statistics are reported in Table 3 for RW, EuroMInd and EuroMInd-C, for the 11 GDP components, and for the combined estimate of GDP (last column). The table also reports the MSFE ratio using EuroMInd-C as the numéraire (with values greater than one indicating that the forecasts are less efficient than EuroMInd-C), as well as the results of the Diebold-Mariano-West test of equal forecasting accuracy. Denoting the forecast error of predictor $(k)$, where $(k)$ is RW or EuroMInd, by $e_{i \tau}^{(k)}=Y_{i \tau}-\hat{Y}_{i \tau}^{(k)}$, that of EuroMind-C by $e_{i \tau}^{(E c)}$, and the quadratic loss differential by $d_{i \tau}=e_{i \tau}^{2(k)}-$ $e_{i \tau}^{2(E c)}$, the Diebold-Mariano-West test of the null hypothesis of equal forecast accuracy, $H_{0}: \mathrm{E}\left(d_{i \tau}\right)=$ 0 , versus the one-sided alternative $H_{1}: \mathrm{E}\left(d_{i \tau}\right)>0$, is the test statistic

$$
D M W_{i}=\frac{\bar{d}_{i}}{\sqrt{\sigma_{i}^{2}}}, \quad \bar{d}_{i}=\frac{1}{M} \sum_{\tau=1}^{M} d_{i \tau}, \quad \sigma_{i}^{2}=\frac{1}{M}\left[c_{0}+2 \sum_{k=1}^{q-1} \frac{q-k}{q} c_{k}\right],
$$

where $c_{k}$ is the sample autocovariance of $d_{i \tau}$ at lag $k$ and $\sigma_{i}^{2}$ is a consistent estimate of the variance of the loss differential. See ? and ?. We set $q=3$ and adopt the DMW statistic with the small sample modification proposed by ?, which corrects for the bias of $\sigma_{i}^{2}$ as an estimator of the variance of $d_{j}(h)$ :

$$
D M W_{i}^{*}=D M W_{i}\left[\frac{J+1-2 q+q(q+1) / J}{J}\right]^{1 / 2} .
$$

The null distribution of the test is Student's $t$ with $M-1$ degrees of freedom.

The results can be summarised as follows:

- The global evidence is that EuroMInd-C produces the most accurate forecasts of the components and GDP: in particular, the forecasts are characterised by less variability and a lower mean absolute percentage error. The relative accuracy (as measured by the MSFE ratio) is greater than one for most of the components of GDP, with the exception of the value added of agriculture (A-B), for which the RW predictor is the most accurate, whereas EuroMInd produces more accurate forecasts for the value added of the sector G-H-I, and for final consumption expenditures and gross capital formation. For combined GDP at market prices, EuroMInd is 7\% less accurate than EuroMInd-C.

- The expenditure components, and GCF, IMP and EXP in particular, are much more difficult to predict; this is reflected in the forecast error variances, which are very large for all of the predictors.

- The Diebold-Mariano-West tests of equal forecast accuracy are never significant as far as the comparison of EuroMInd and EuroMInd-C is concerned.

- EuroMInd-C significantly outperforms the RW predictor: the Diebold-Mariano-West test leads to a strong rejection for the combined GDP prediction and some GDP components.

\subsection{Parameter instability}

The recursive forecasting experiment enables us to assess the variability of the parameter estimates according to the sample period considered. Let $\tilde{\boldsymbol{\Xi}}_{\tau}$ denote the parameter estimates obtained in quarter 
Table 3: Comparative assessment of the predictive performances of EuroMInd-C and EuroMInd in real time. RW refers to the random walk or "no change" predictor.

Mean error

\begin{tabular}{lrrrrrrrrrrrr}
\hline Models & A-B & C-D-E & F & G-H-I & J-K & L-P & TlS & FCE & GCF & EXP & IMP & GDP \\
\hline RW & 0.10 & 0.50 & -0.30 & 0.81 & 1.31 & 2.07 & 0.03 & 3.37 & -0.36 & 7.24 & 5.81 & 4.50 \\
EuroMInd & -0.18 & 0.20 & -0.33 & -0.55 & -0.02 & 0.05 & -0.50 & -2.25 & -2.02 & -0.15 & -1.58 & -1.56 \\
EuroMInd-C & -0.16 & -0.83 & -0.52 & -1.38 & -0.29 & -1.28 & -0.71 & -3.31 & -3.38 & -5.03 & -5.78 & -5.32
\end{tabular}

Variance

\begin{tabular}{lrrrrrrrrrrrr}
\hline Models & A-B & C-D-E & F & G-H-I & J-K & L-P & TlS & FCE & GCF & EXP & IMP & GDP \\
\hline RW & 0.21 & 78.59 & 1.86 & 8.90 & 2.03 & 9.03 & 4.41 & 20.81 & 147.87 & 521.17 & 382.39 & 265.26 \\
EuroMInd & 0.55 & 40.12 & 1.77 & 5.77 & 1.89 & 9.51 & 4.52 & 16.54 & 109.56 & 308.84 & 264.15 & 129.23 \\
EuroMInd-C & 0.47 & 22.44 & 1.42 & 5.55 & 1.72 & 7.00 & 3.85 & 17.34 & 109.79 & 277.83 & 211.85 & 94.26
\end{tabular}

Mean square forecast error

\begin{tabular}{lrrrrrrrrrrrr}
\hline Models & A-B & C-D-E & F & G-H-I & J-K & L-P & TlS & FCE & GCF & EXP & IMP & GDP \\
\hline RW & 0.22 & 78.84 & 1.95 & 9.55 & 3.73 & 13.34 & 4.41 & 32.18 & 148.00 & 573.58 & 416.14 & 285.53 \\
EuroMInd & 0.58 & 40.16 & 1.88 & 6.07 & 1.89 & 9.52 & 4.77 & 21.62 & 113.64 & 308.86 & 266.63 & 131.65 \\
EuroMInd-C & 0.49 & 23.13 & 1.69 & 7.45 & 1.81 & 8.64 & 4.35 & 28.29 & 121.20 & 303.18 & 245.30 & 122.61
\end{tabular}

Mean absolute error

\begin{tabular}{lrrrrrrrrrrrr}
\hline Models & A-B & C-D-E & F & G-H-I & J-K & L-P & TlS & FCE & GCF & EXP & IMP & GDP \\
\hline RW & 0.34 & 5.97 & 1.22 & 2.57 & 1.41 & 3.01 & 1.51 & 4.66 & 9.27 & 18.43 & 16.29 & 13.37 \\
EuroMInd & 0.46 & 3.77 & 1.14 & 1.69 & 1.10 & 2.35 & 1.62 & 3.94 & 8.42 & 12.02 & 11.58 & 7.50 \\
EuroMInd-C & 0.41 & 2.73 & 1.04 & 1.83 & 0.97 & 2.00 & 1.46 & 4.08 & 8.44 & 9.98 & 10.77 & 6.90
\end{tabular}

Mean absolute percentage error

\begin{tabular}{lrrrrrrrrrrrr}
\hline Models & A-B & C-D-E & F & G-H-I & J-K & L-P & TlS & FCE & GCF & EXP & IMP & GDP \\
\hline RW & 0.90 & 1.70 & 1.30 & 0.77 & 0.79 & 0.43 & 0.81 & 0.32 & 2.38 & 2.22 & 2.03 & 0.71 \\
EuroMInd & 1.25 & 1.07 & 1.23 & 0.50 & 0.61 & 0.33 & 0.87 & 0.27 & 2.17 & 1.44 & 1.45 & 0.40 \\
EuroMInd-C & 1.12 & 0.77 & 1.12 & 0.55 & 0.53 & 0.28 & 0.78 & 0.28 & 2.18 & 1.20 & 1.35 & 0.36
\end{tabular}

Relative MSFE (with respect to EuroMInd-C)

\begin{tabular}{lrrrrrrrrrrrr}
\hline Models & A-B & C-D-E & F & G-H-I & J-K & L-P & TIS & FCE & GCF & EXP & IMP & GDP \\
\hline RW & 0.45 & 3.41 & 1.16 & 1.28 & 2.06 & 1.54 & 1.01 & 1.14 & 1.22 & 1.89 & 1.70 & 2.33 \\
EuroMInd & 1.18 & 1.74 & 1.11 & 0.82 & 1.04 & 1.10 & 1.10 & 0.76 & 0.94 & 1.02 & 1.09 & 1.07
\end{tabular}

Diebold-Mariano-West statistic (versus EuroMInd-C)

\begin{tabular}{lrrrrrrrrrrrr}
\hline Models & A-B & C-D-E & F & G-H-I & J-K & L-P & TlS & FCE & GCF & EXP & IMP & GDP \\
\hline RW & 0.62 & 1.48 & 0.91 & 1.16 & 1.82 & 1.29 & -0.20 & 0.44 & 1.33 & 2.80 & 2.88 & 2.38 \\
EuroMInd & 0.67 & 1.75 & 0.64 & -1.54 & 0.24 & 0.92 & 0.97 & -1.61 & -0.79 & 0.10 & 0.74 & 0.90
\end{tabular}




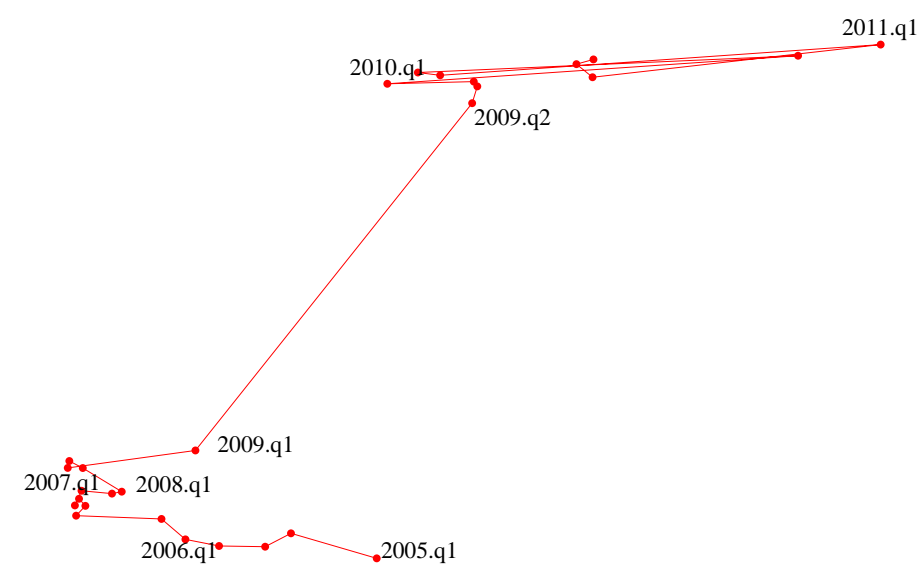

Figure 7: Metric scaling analysis of recursive parameter estimates. Each point represents the parameter estimate $\tilde{\boldsymbol{\Xi}}_{\tau}$ obtained from the release available at quarter $\tau=2005 . q 1, \ldots, 2011 . q 4$.

$\tau, \tau=2005 . q 1, \ldots, 2011 . q 4$; a measure of parameter instability with estimation period $\tau^{\prime}$ should be based on the Mahalanobis distance between the vectors $\tilde{\boldsymbol{\Xi}}_{\tau}$ and $\tilde{\boldsymbol{\Xi}}_{\tau^{\prime}}$, for $\tau^{\prime} \neq \tau$. The latter is a weighted Euclidean distance, where the weighting matrix is the average precision matrix,

$$
d\left(\tau, \tau^{\prime}\right)=\left(\tilde{\boldsymbol{\Xi}}_{\tau}-\tilde{\boldsymbol{\Xi}}_{\tau^{\prime}}\right)^{\prime}[\operatorname{Var}(\tilde{\boldsymbol{\Xi}})]^{-1}\left(\tilde{\boldsymbol{\Xi}}_{\tau}-\tilde{\boldsymbol{\Xi}}_{\tau^{\prime}}\right) .
$$

This measure requires the precision matrix $[\operatorname{Var}(\tilde{\boldsymbol{\Xi}})]^{-1}$, which is not made available by the EM algorithm.

However, a descriptive assessment of parameter instability can be obtained from the spectral decomposition of the correlation matrix of the parameter estimates $\tilde{\boldsymbol{\Xi}}_{\tau}, \tau=2005 . q 1, \ldots, 2011 . q 4$, by plotting the first two eigenvectors scaled by the square root of the corresponding eigenvalue. This amounts to performing a metric scaling analysis of the matrix stacking the vectors $\tilde{\Xi}_{\tau}^{\prime}($ see ?).

This is done in Figure 7, where each point represents the parameter estimate at a particular point in time and the Euclidean distance between the points is the best rank 2 approximation to the Mahalanobis distance $d\left(\tau, \tau^{\prime}\right)$. The approximation is good, as the first two eigenvalues account for $98 \%$ of the original total variation.

The most prominent fact presented by the figure is the rather large shift in the parameter estimates that occurs with the release $\tau=2009 . q 2$, which uses the data up to May 2009. It ought to be mentioned the real effects of the global financial crisis hit the Euro area around the end of 2008; thus, the main factor driving the parameter change along the vertical dimension of the graph is the strong recessionary movement resulting from the global financial crisis. A second important fact is that a common factor (determining the horizontal dimension of the plot and accounting for $94 \%$ of the total variation) drives the change in the parameters.

We did not address the important issue of assessing whether or not the estimation of structural change via the inclusion of suitable interventions helps to improve the accuracy of EuroMInd-C in forecasting and signal extraction, along the lines indicated by ?. This represents an interesting topic for further research in this area. 


\subsection{Comparison with other coincident indicators of economic growth}

We have already compared EuroMInd-C with EuroMInd in terms of predictive accuracies. Another consideration is that EuroMInd-C provides monthly indicators of the 11 GDP components for four individual countries along with the Euro area; furthermore, within the estimation sample, the estimated components are more informative, as they embody more information. This shows up in a higher reliability of the smoothed, or historical, estimates, as is reflected by a lower estimation standard error.

Several alternative monthly indicators of economic activity have been constructed with objectives similar to that of EuroMInd-C; this subsection provides a qualitative assessment of their different information contents. Among these indicators, New Eurocoin (NE) and the Economic Sentiment Index (ESI) are prominent.

New Eurocoin is a monthly coincident indicator of economic growth for the Euro area (?) that is published monthly by CEPR (www.cepr.org) and the Bank of Italy. The indicator is a measure of the quarterly GDP underlying growth, devoid of short run fluctuations (those with a periodicity of less than one year), based on its linear projection on the space of the relevant dynamic principal components, constructed on a set of about 150 transformed monthly time series.

The first panel of Figure 8 compares NE with the quarterly logarithmic changes of EuroMInd-C. There are several differences:

1. NE is smoother than EuroMInd-C. This is because EuroMInd-C does not impose smoothness priors.

2. NE is an estimate of the GDP underlying growth, devoid of short-run fluctuations with a periodicity of less than one year (medium to long run growth), assuming a quarterly horizon for growth. EuroMInd-C does not assume any particular horizon and can be used for the assessment of growth over any horizon.

3. The amplitude of the fluctuations and the characterisation of the quarterly growth rate cycle differ: $\mathrm{NE}$ ascribes a larger cyclical amplitude to the fluctuations before the global financial crisis, and the sharpness of the trough and the subsequent recovery during 2009 are very different.

4. NE does not enforce the temporal aggregation constraints and uses a different interpolation scheme. EuroMInd-C estimates a monthly level of GDP that is consistent with the quarterly totals.

5. There are also other methodological differences and the elementary series considered differ in part. For instance, the consumer and producer price series do not play any role in the construction of EuroMInd-C.

The European Commission (Directorate-General for Economic and Financial Affairs) compiles the Economic Sentiment Indicator (ESI), a composite coincident indicator for the timely assessment of the socio-economic situation in the Euro area. ESI is computed by aggregating the seasonally adjusted balances of opinions to a selection of qualitative survey questions from five sectors (industry, services, construction, retail trade and consumers) related to reference "hard" variables, such as production in industry.

We have found the most appropriate comparison to be with the yearly changes in EuroMInd-C, which are displayed in the second panel of Figure 8 and compared to the rescaled ESI. Despite the apparent similarity of the series, there are notable differences in the locations of the peaks and troughs of the annual growth rate cycle and in the speed of the recovery occurring around the years 2009-2010. In particular, ESI provided a much more optimistic assessment of the recovery than appeared in the monthly 
GDP estimates. While the comparison seems to establish that ESI tracks economic growth at a yearly horizon, we should mention that EuroMInd-C embodies a supervised approach that links the indicator to the national account series. On the other hand, the ESI methodology is 'unsupervised', in that only the balances of opinions are used for the estimation of the indicator. As a result, the interpretation of the scale of the indicator is not related to GDP growth.

This qualitative assessment, coupled with the considerations arising from the rolling forecast evaluation, suggests that EuroMInd-C is a useful addition to the set of coincident indicators that is available for the Euro area and its main economies.

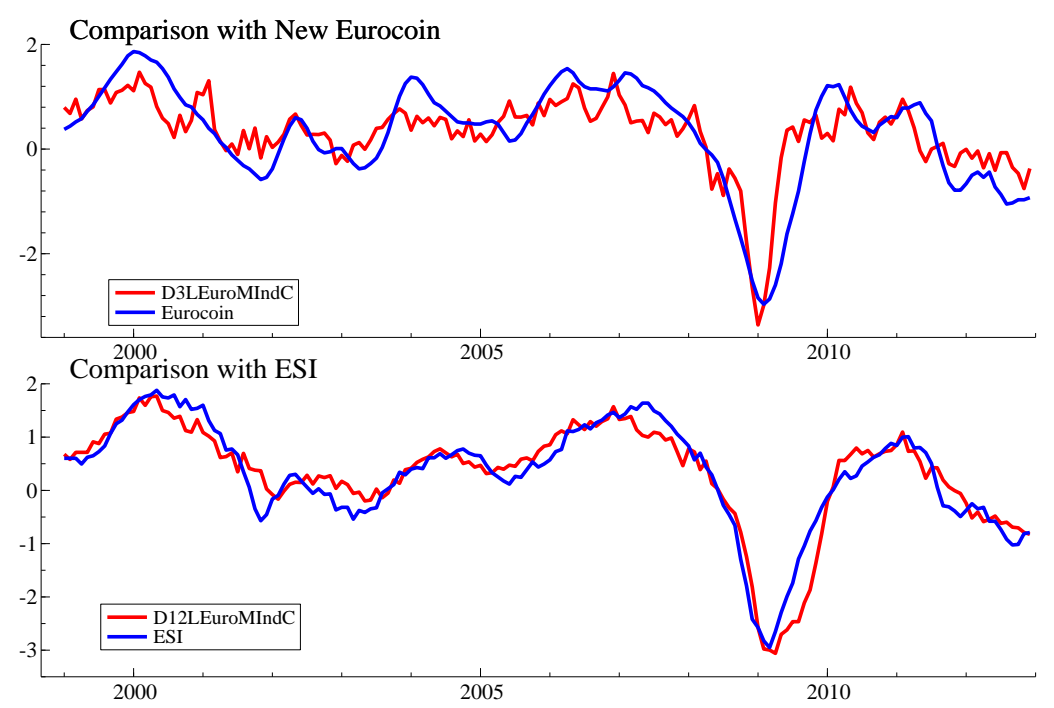

Figure 8: Comparison of EuroMInd with New Eurocoin and the Economic Sentiment Indicator. 


\section{Conclusions}

In this paper we have introduced EuroMInd-C, a set of monthly indicators of GDP for the Euro area and its four largest economies that are relevant, representative and timely. The estimates can be used to assess the economic stance in a timely manner, and allow an appreciation of both the broad picture (Euro area) and the country- and sector-specific details.

The characteristic element of EuroMInd-C that distinguishes it from other economic indicators based on dynamic factor models is the centrality of the national accounts framework, which provides the backbone of the system, but also adds complexity to it.

We have illustrated the methodological issues that arise when estimating a large scale dynamic factor model that is subject to both temporal aggregation constraints and generic patterns of missing observations, and documented the technical solutions that can be adopted in the state space framework.

For real time implementation, batch estimation of the resulting mixed frequency factor model is feasible, albeit computationally demanding. Hence, we envisage a strategy that performs the estimation of the hyperparameters once each semester or year, while filtering and smoothing can be performed in real time each time the dataset is updated.

A pseudo real-time recursive forecasting experiment has provided evidence that EuroMInd-C can be more accurate for predicting GDP than our previous componentwise approach, implemented in the EuroMInd indicator (see ?). Moreover, the comparative assessment with other coincident indicators suggests that EuroMInd-C is a useful addition to the set of coincident indicators that is available for the Euro area and its main economies.

To conclude, we note that the methodology implemented in this paper can also be used in other contexts that require the information in unbalanced multi-dimensional datasets that are subject to sectional and temporal aggregation constraints to be modelled and summarized.

\section{Acknowledgements}

The authors wish to thank the reviewers and the Editor for their careful reviews and constructive criticism that led to several improvements in the paper. Stefano Grassi and Tommaso Proietti gratefully acknowledge support from CREATES: Center for Research in Econometric Analysis of Time Series (DNRF78), funded by the Danish National Research Foundation. Tommaso Proietti acknowledges financial support by the Italian Ministry of Education, University and Research (MIUR), PRIN Research Project 2010-2011: prot. 2010J3LZEN, Forecasting economic and financial time series: understanding the complexity and modelling structural change.

\section{Appendix A: List of series in the dataset}

The time series used for the estimation of EuroMInd-C were downloaded from the Europa dataset, which is made available electronically by Eurostat on the web site http://epp.eurostat.ec.europa. $\mathrm{eu} /$. The series of value added and GDP components are published with 65-day delays with respect to the end of each quarter, according to a release calendar that is available at the beginning of each year. However, a preliminary estimate of GDP at market prices, the so-called flash estimate, is released in advance ( 45 days after the end of each quarter), with the aim of providing a more timely assessment 
of the level of economic activity. Of the monthly indicators, the most timely series are the business and consumer survey data, which are made available at the end of each month, along with the financial aggregates compiled by the ECB (around 30-day delays), whereas the data for the industry sector and retail (such as car registrations by OECD) are published about 45 days after the end of the reference month. Other indicators, such as those for construction (index of production and building permits), and the series for the agricultural sector and the labour market (employment and hours worked) have publication delays of about 70 days. Finally, due to the complexity of data collection process, the series relating to external trade are made available with a longer delay, amounting to about 90 days for the volume index of exports and imports.

Table 4 provides the complete list by GDP component, using the Europa dataset labels. It also reports the time spans of the series and the numbers of countries available for each series. Finally, the Euro area is the one defined by 17 countries.

\section{Appendix B: Initial conditions}

As was stated above, the state space formulation is modified when there are missing values that require adjustment at the beginning of the sample. The initial two observations, $t=0,1$, have a special representation. At time $t=0$, we have the following measurement equation:

$$
\boldsymbol{Y}_{10}^{c}=\left[\begin{array}{llllll}
\boldsymbol{I}_{N_{1}} & \mathbf{0} & \mathbf{0} & \mathbf{0} & \mathbf{0} & \mathbf{0}
\end{array}\right] \boldsymbol{\alpha}_{0},
$$

where the initial state vector is defined as

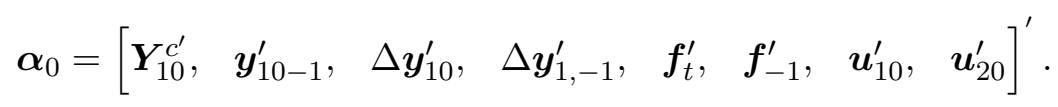

The specification of the model requires the specification of the distribution of the initial state vector $\boldsymbol{\alpha}_{0}$. For this purpose, the first block is rewritten as $\boldsymbol{Y}_{1,0}^{c}=\exp \left(\boldsymbol{y}_{1,0}\right)$, for $\rho_{0}=0$; its first order Taylor approximation around the trial value $\boldsymbol{y}_{1,0}^{*}$ is

$$
\begin{aligned}
\boldsymbol{Y}_{1,0}^{c} & =\exp \left(\boldsymbol{y}_{1,0}^{*}\right)+\boldsymbol{U}_{1,0}^{*} \boldsymbol{y}_{1,0}-\boldsymbol{U}_{1,0}^{*} \boldsymbol{y}_{1,0}^{*} \\
& =\left(\exp \left(\boldsymbol{y}_{1,0}^{*}\right)+\boldsymbol{U}_{1,0}^{*} \boldsymbol{\mu}_{1}-\boldsymbol{U}_{1,0}^{*} \boldsymbol{y}_{1,0}^{*}\right)+\boldsymbol{U}_{1,0}^{*} \boldsymbol{y}_{1,-1}+\boldsymbol{U}_{1,0}^{*} \Theta_{1} \boldsymbol{f}_{0}+\boldsymbol{U}_{1,0}^{*} \boldsymbol{u}_{1,0} .
\end{aligned}
$$

The first two blocks of the state vector are nonstationary and are initialised by the vector $\boldsymbol{\beta}=\boldsymbol{y}_{1,-1}$, whereas the last four blocks have stationary distributions, which depend on $\boldsymbol{f}_{0} \sim \mathrm{N}\left(\mathbf{0}, \boldsymbol{\Sigma}_{f}\right)$, where $\boldsymbol{\Sigma}_{f}$ solves the matrix equation $\boldsymbol{\Sigma}_{f}=\boldsymbol{\Phi} \boldsymbol{\Sigma}_{f} \boldsymbol{\Phi}^{\prime}+\boldsymbol{\Sigma}_{\eta}$, and $\boldsymbol{u}_{0}=\left[\boldsymbol{u}_{1,0}^{\prime}, \boldsymbol{u}_{2,0}^{\prime}\right]^{\prime} \sim \mathrm{N}\left(\mathbf{0}, \boldsymbol{\Sigma}_{u}\right)$, where $\boldsymbol{\Sigma}_{u}$ solves the matrix equation $\boldsymbol{\Sigma}_{u}=\boldsymbol{\Psi} \boldsymbol{\Sigma}_{\epsilon} \boldsymbol{\Psi}^{\prime}+\boldsymbol{\Sigma}_{\epsilon}$.

Since $\boldsymbol{y}_{1,0}=\boldsymbol{y}_{1,-1}+\Delta \boldsymbol{y}_{1,0}$, the initial state vector is thus written as:

$$
\boldsymbol{\alpha}_{0}=\boldsymbol{A}_{0,0} \boldsymbol{\beta}+\boldsymbol{a}_{0,0}+\boldsymbol{H}_{0} \boldsymbol{\omega}_{0}, \quad \boldsymbol{\omega}_{0}=\left[\begin{array}{c}
\boldsymbol{f}_{0} \\
\boldsymbol{f}_{-1} \\
\boldsymbol{u}_{10} \\
\boldsymbol{u}_{20} \\
\boldsymbol{u}_{1,-1}
\end{array}\right]
$$




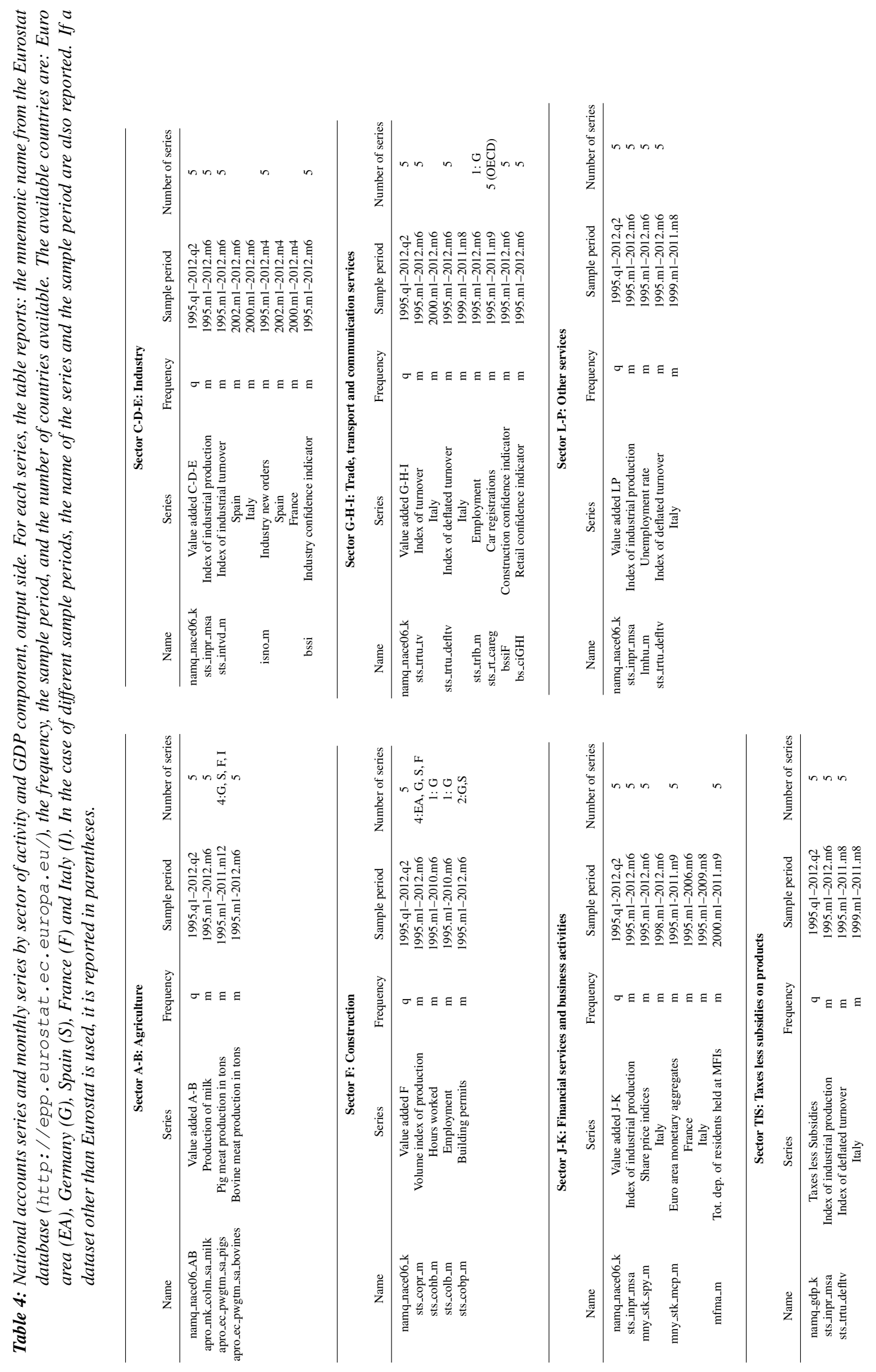




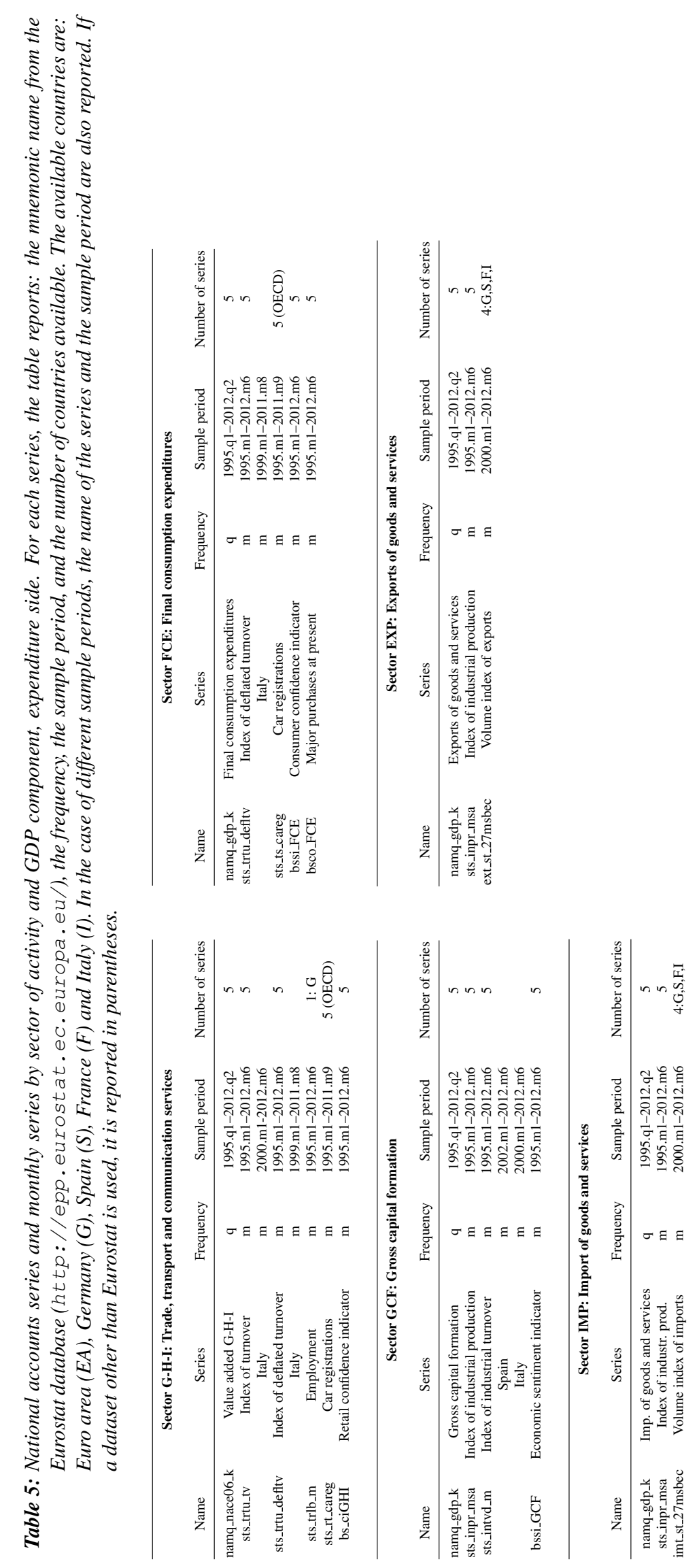


where

$$
\begin{gathered}
\boldsymbol{a}_{0,0}=\left[\begin{array}{c}
\boldsymbol{Y}_{1,0}^{c *}-\boldsymbol{U}_{1,0}^{*} \boldsymbol{y}_{1,0}^{*}+\boldsymbol{U}_{1,0}^{*} \boldsymbol{\mu}_{1} \\
\mathbf{0} \\
\boldsymbol{\mu}_{1} \\
\boldsymbol{\mu}_{1} \\
\mathbf{0} \\
\mathbf{0} \\
\mathbf{0}
\end{array}\right], \quad \boldsymbol{A}_{0,0}=\left[\begin{array}{c}
\boldsymbol{U}_{1,0}^{*} \\
\boldsymbol{I}_{N 1} \\
\mathbf{0} \\
\mathbf{0} \\
\mathbf{0} \\
\end{array}\right], \quad \boldsymbol{P}_{0,0}=\boldsymbol{H}_{0} \operatorname{Cov}\left(\boldsymbol{\omega}_{0}\right) \boldsymbol{H}_{0}^{\prime} . \\
\boldsymbol{H}_{0}
\end{gathered}
$$

We assume that $\boldsymbol{\beta}$ is a diffuse random vector, i.e., it has an improper distribution with zero mean and an arbitrarily large variance matrix. The diffuse case captures the nonstationarity of a particular unobserved component, and entails marginalising the inferences with respect to the parameter vector $\boldsymbol{\beta}$. As ? has shown, the posterior mean of $\boldsymbol{\beta}$ under the diffuse prior is coincident with the generalised least squares estimate of the parameter $\boldsymbol{\beta}$, considered as a fixed parameter vector in the classical sense.

At time $t=1$, the measurement equation becomes:

$$
\left[\begin{array}{c}
\boldsymbol{Y}_{11}^{c} \\
\Delta \boldsymbol{y}_{21}
\end{array}\right]=\left[\begin{array}{cccccccc}
\boldsymbol{I}_{N_{1}} & \mathbf{0} & \mathbf{0} & \mathbf{0} & \mathbf{0} & \mathbf{0} & \mathbf{0} & \mathbf{0} \\
\mathbf{0} & \mathbf{0} & \mathbf{0} & \mathbf{0} & \boldsymbol{\Theta}_{2} & \mathbf{0} & \mathbf{0} & \boldsymbol{I}_{N_{2}}
\end{array}\right] \boldsymbol{\alpha}_{1}+\left[\begin{array}{c}
\mathbf{0} \\
\boldsymbol{\mu}_{2}
\end{array}\right]+\left[\begin{array}{ccc}
\mathbf{0} & \mathbf{0} & \mathbf{0} \\
\mathbf{0} & \mathbf{0} & \mathbf{0}
\end{array}\right]\left[\begin{array}{c}
\boldsymbol{\eta}_{t} \\
\boldsymbol{\epsilon}_{1 t} \\
\boldsymbol{\epsilon}_{2 t}
\end{array}\right]
$$

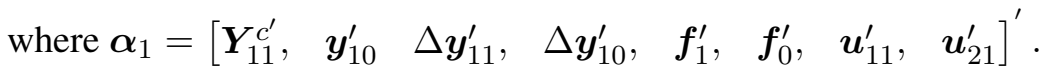


The transition equation at time $t=1$ is

$$
\begin{aligned}
& {\left[\begin{array}{c}
\boldsymbol{Y}_{1 t}^{c} \\
\boldsymbol{y}_{1 t-1} \\
\Delta \boldsymbol{y}_{1 t} \\
\Delta \boldsymbol{y}_{1 t-1} \\
\boldsymbol{f}_{t} \\
\boldsymbol{f}_{t-1} \\
\boldsymbol{u}_{1 t} \\
\boldsymbol{u}_{2 t}
\end{array}\right]=\left[\begin{array}{cccccccc}
\rho_{1} \boldsymbol{I}_{N 1} & \boldsymbol{U}_{11}^{*} & \boldsymbol{U}_{11}^{*}\left(\boldsymbol{I}+\boldsymbol{\Psi}_{1}\right) & \mathbf{0} & \boldsymbol{U}_{11}^{*}\left[\boldsymbol{\Theta}_{1} \boldsymbol{\Phi}-\boldsymbol{\Psi}_{1} \boldsymbol{\Theta}_{1}\right] & \mathbf{0} & \mathbf{0} & \mathbf{0} \\
\mathbf{0} & \boldsymbol{I}_{N 1} & \boldsymbol{I}_{N 1} & \mathbf{0} & \mathbf{0} & \mathbf{0} & \mathbf{0} & \mathbf{0} \\
\mathbf{0} & \mathbf{0} & \boldsymbol{\Psi}_{N_{1}} & \mathbf{0} & {\left[\boldsymbol{\Theta}_{1} \boldsymbol{\Phi}-\boldsymbol{\Psi}_{1} \boldsymbol{\Theta}_{1}\right]} & \mathbf{0} & \mathbf{0} & \mathbf{0} \\
\mathbf{0} & \mathbf{0} & \boldsymbol{I} & \mathbf{0} & \mathbf{0} & \mathbf{0} & \mathbf{0} & \mathbf{0} \\
\mathbf{0} & \mathbf{0} & \mathbf{0} & \mathbf{0} & \mathbf{\Phi} & \mathbf{0} & \mathbf{0} & \mathbf{0} \\
\mathbf{0} & \mathbf{0} & \mathbf{0} & \mathbf{0} & \boldsymbol{I} & \mathbf{0} & \mathbf{0} & \mathbf{0} \\
\mathbf{0} & \mathbf{0} & \mathbf{0} & \mathbf{0} & \mathbf{0} & \mathbf{0} & \boldsymbol{\Psi}_{N_{1}} & \mathbf{0} \\
\mathbf{0} & \mathbf{0} & \mathbf{0} & \mathbf{0} & \mathbf{0} & \mathbf{0} & \mathbf{0} & \boldsymbol{\Psi}_{N_{2}}
\end{array}\right]\left[\begin{array}{c}
\boldsymbol{Y}_{10}^{c} \\
\boldsymbol{y}_{1-1} \\
\Delta \boldsymbol{y}_{10} \\
\Delta \boldsymbol{y}_{1-1} \\
\boldsymbol{f}_{0} \\
\boldsymbol{f}_{-1} \\
\boldsymbol{u}_{10} \\
\boldsymbol{u}_{20}
\end{array}\right]} \\
& +\left[\begin{array}{c}
\boldsymbol{Y}_{1 t}^{*}-\boldsymbol{U}_{1 t}^{*} \boldsymbol{y}_{1 t}^{*}+\boldsymbol{U}_{1 t}^{*}\left(\boldsymbol{I}-\boldsymbol{\Psi}_{N_{1}}\right) \boldsymbol{\delta}_{1} \\
\mathbf{0} \\
\left(\boldsymbol{I}-\boldsymbol{\Psi}_{N_{1}}\right) \boldsymbol{\delta}_{1} \\
\mathbf{0} \\
\mathbf{0} \\
\mathbf{0} \\
\mathbf{0}
\end{array}\right]+\left[\begin{array}{ccc}
\boldsymbol{U}_{1 t} \boldsymbol{\Theta}_{1} & \boldsymbol{U}_{1 t}^{*} & \mathbf{0} \\
\mathbf{0} & \mathbf{0} & \mathbf{0} \\
\boldsymbol{\Theta}_{1} & \boldsymbol{I}_{N_{1}} & \mathbf{0} \\
\mathbf{0} & \mathbf{0} & \mathbf{0} \\
\boldsymbol{I}_{k} & \mathbf{0} & \mathbf{0} \\
\mathbf{0} & \mathbf{0} & \mathbf{0} \\
\mathbf{0} & \boldsymbol{I}_{N_{1}} & \mathbf{0} \\
\mathbf{0} & \mathbf{0} & \boldsymbol{I}_{N_{2}}
\end{array}\right]\left[\begin{array}{c}
\boldsymbol{\eta}_{t} \\
\boldsymbol{\epsilon}_{1 t} \\
\boldsymbol{\epsilon}_{2 t}
\end{array}\right] .
\end{aligned}
$$

\section{Appendix C: The treatment of missing values}

\section{Case 1: Missing at time $t$ but observed at time $t-1$}

The state vector needs to be augmented by two components for each missing value affecting $\boldsymbol{y}_{t}$ at time $t$ becoming $\boldsymbol{\alpha}_{t}=\left[\boldsymbol{Y}_{1 t}^{c^{\prime}}, \boldsymbol{y}_{1 t-1}^{\prime}, \Delta \boldsymbol{y}_{1 t}^{\prime}, \Delta \boldsymbol{y}_{1 t-1}^{\prime}, \boldsymbol{f}_{t}^{\prime}, \boldsymbol{f}_{t-1}^{\prime}, \boldsymbol{\alpha}_{1 t}^{+^{\prime}}, \boldsymbol{\alpha}_{2 t}^{+^{\prime}}\right]^{\prime}$, where the $\boldsymbol{\alpha}_{1 t}^{+^{\prime}}, \boldsymbol{\alpha}_{2 t}^{+^{\prime}}$ are latent states that are used to take the missing values into account. The measurement equation is modified as follows:

$$
\left[\begin{array}{c}
\boldsymbol{Y}_{1 t}^{c} \\
\Delta \boldsymbol{y}_{2 t}
\end{array}\right]=\left[\begin{array}{cccccccc}
\boldsymbol{I}_{N_{1}} & \mathbf{0} & \mathbf{0} & \mathbf{0} & \mathbf{0} & \mathbf{0} & \mathbf{0} & \mathbf{0} \\
\mathbf{0} & \mathbf{0} & \mathbf{0} & \mathbf{0} & \boldsymbol{\Theta}_{2} & -\boldsymbol{\Psi}_{2} \boldsymbol{\Theta}_{2} & \mathbf{0} & \mathbf{0}
\end{array}\right] \boldsymbol{\alpha}_{t}+\left[\begin{array}{c}
\mathbf{0} \\
\left(\boldsymbol{I}-\boldsymbol{\Psi}_{2}\right) \boldsymbol{\delta}_{2}
\end{array}\right]+\left[\begin{array}{lll}
\mathbf{0} & \mathbf{0} & \mathbf{0} \\
\mathbf{0} & \mathbf{0} & \boldsymbol{I}
\end{array}\right]\left[\begin{array}{c}
\boldsymbol{\eta}_{t} \\
\boldsymbol{\epsilon}_{1 t} \\
\boldsymbol{\epsilon}_{2 t}
\end{array}\right],
$$


and the state equation becomes:

$$
\begin{aligned}
& {\left[\begin{array}{c}
\boldsymbol{Y}_{1 t}^{c} \\
\boldsymbol{y}_{1 t-1} \\
\Delta \boldsymbol{y}_{1 t} \\
\Delta \boldsymbol{y}_{1 t-1} \\
\boldsymbol{f}_{t} \\
\boldsymbol{f}_{t-1} \\
\boldsymbol{\alpha}_{1 t}^{+} \\
\boldsymbol{\alpha}_{2 t}^{+}
\end{array}\right]=\left[\begin{array}{cccccc}
\rho_{t} \boldsymbol{I}_{N 1} & \boldsymbol{U}_{1 t}^{*} & \boldsymbol{U}_{1 t}^{*}\left(\boldsymbol{I}+\boldsymbol{\Psi}_{1}\right) & \mathbf{0} & \boldsymbol{U}_{1 t}^{*}\left[\boldsymbol{\Theta}_{1} \boldsymbol{\Phi}-\boldsymbol{\Psi}_{1} \boldsymbol{\Theta}_{1}\right] & \mathbf{0} \\
\mathbf{0} & \boldsymbol{I}_{N 1} & \boldsymbol{I}_{N 1} & \mathbf{0} & \mathbf{0} & \mathbf{0} \\
\mathbf{0} & \mathbf{0} & \boldsymbol{\Psi}_{N} & \mathbf{0} & {\left[\boldsymbol{\Theta}_{1} \boldsymbol{\Phi}-\boldsymbol{\Psi}_{1} \boldsymbol{\Theta}_{1}\right]} & \mathbf{0} \\
\mathbf{0} & \mathbf{0} & \boldsymbol{I} & \mathbf{0} & \mathbf{0} & \mathbf{0} \\
\mathbf{0} & \mathbf{0} & \mathbf{0} & \mathbf{0} & \mathbf{\Phi} & \mathbf{0} \\
\mathbf{0} & \mathbf{0} & \mathbf{0} & \mathbf{0} & \boldsymbol{I} & \mathbf{0} \\
\mathbf{0} & \mathbf{0} & \mathbf{0} & \mathbf{0} & {\left[\boldsymbol{\Theta}_{2} \mathbf{\Phi}-\boldsymbol{\Psi}_{2} \boldsymbol{\Theta}_{2}\right]} & \mathbf{0} \\
\mathbf{0} & \mathbf{0} & \mathbf{0} & \mathbf{0} & {\left[\boldsymbol{\Theta}_{2} \mathbf{\Phi}-\mathbf{\Psi}_{2} \boldsymbol{\Theta}_{2}\right]} & \mathbf{0}
\end{array}\right]\left[\begin{array}{c}
\boldsymbol{Y}_{1 t-1}^{c} \\
\boldsymbol{y}_{1 t-2} \\
\Delta \boldsymbol{y}_{1 t-1} \\
\Delta \boldsymbol{y}_{1 t-2} \\
\boldsymbol{f}_{t-1} \\
\boldsymbol{f}_{t-2}
\end{array}\right]}
\end{aligned}
$$

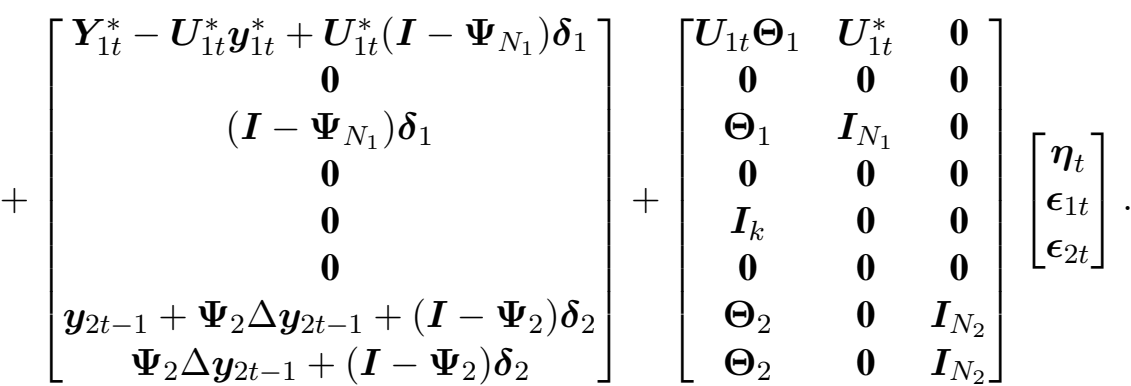

$\Delta \boldsymbol{y}_{2 t}$ corresponds to the series that are available at times $t$ and $t-1$. The missing series do not enter the calculation of the likelihood, but they are propagated in the latent states using $\boldsymbol{\alpha}_{1 t}^{+{ }^{\prime}}, \boldsymbol{\alpha}_{2 t}^{+\prime}$. The available observations for the series that are missing at time $t$ but observed at time $t-1$ are inserted in the latent states using the relationship $\boldsymbol{y}_{2 t-1}+\boldsymbol{\Psi}_{2} \Delta \boldsymbol{y}_{2 t-1}+\left(\boldsymbol{I}-\boldsymbol{\Psi}_{2}\right) \boldsymbol{\delta}_{2}$, where $\boldsymbol{y}_{2 t-1}$ represents series in levels observed at time $t-1$ and $\Delta \boldsymbol{y}_{2 t-1}$ represents series in first differences at time $t-1$.

\section{Case 2: Missing at time $t$ and time $t-1$}

As before, $\Delta \boldsymbol{y}_{2 t}$ corresponds to the available observations at time $t$ and $t-1$. The observations that are still missing are propagated using the $\boldsymbol{\alpha}_{1 t}^{+^{\prime}}, \boldsymbol{\alpha}_{2 t}^{+^{\prime}}$ created in Case 1. The measurement equation is modified as follows:

$$
\left[\begin{array}{c}
\boldsymbol{Y}_{1 t}^{c} \\
\Delta \boldsymbol{y}_{2 t}
\end{array}\right]=\left[\begin{array}{cccccccc}
\boldsymbol{I}_{N_{1}} & \mathbf{0} & \mathbf{0} & \mathbf{0} & \mathbf{0} & \mathbf{0} & \mathbf{0} & \mathbf{0} \\
\mathbf{0} & \mathbf{0} & \mathbf{0} & \mathbf{0} & \boldsymbol{\Theta}_{2} & -\boldsymbol{\Psi}_{2} \boldsymbol{\Theta}_{2} & \mathbf{0} & \mathbf{0}
\end{array}\right] \boldsymbol{\alpha}_{t}+\left[\begin{array}{c}
\mathbf{0} \\
\left(\boldsymbol{I}-\boldsymbol{\Psi}_{2}\right) \boldsymbol{\delta}_{2}
\end{array}\right]+\left[\begin{array}{lll}
\mathbf{0} & \mathbf{0} & \mathbf{0} \\
\mathbf{0} & \mathbf{0} & \boldsymbol{I}
\end{array}\right]\left[\begin{array}{c}
\boldsymbol{\eta}_{t} \\
\boldsymbol{\epsilon}_{1 t} \\
\boldsymbol{\epsilon}_{2 t}
\end{array}\right]
$$


and the state equation becomes:

$$
\begin{aligned}
& {\left[\begin{array}{c}
\boldsymbol{Y}_{1 t}^{c} \\
\boldsymbol{y}_{1 t-1} \\
\Delta \boldsymbol{y}_{1 t} \\
\Delta \boldsymbol{y}_{1 t-1} \\
\boldsymbol{f}_{t} \\
\boldsymbol{f}_{t-1} \\
\boldsymbol{\alpha}_{1 t}^{+} \\
\boldsymbol{\alpha}_{2 t}^{+}
\end{array}\right]=\left[\begin{array}{cccccccc}
\rho_{t} \boldsymbol{I}_{N 1} & \boldsymbol{U}_{1 t}^{*} & \boldsymbol{U}_{1 t}^{*}\left(\boldsymbol{I}+\boldsymbol{\Psi}_{1}\right) & \mathbf{0} & \boldsymbol{U}_{1 t}^{*}\left[\boldsymbol{\Theta}_{1} \boldsymbol{\Phi}-\boldsymbol{\Psi}_{1} \boldsymbol{\Theta}_{1}\right] & \mathbf{0} & \mathbf{0} & \mathbf{0} \\
\mathbf{0} & \boldsymbol{I}_{N 1} & \boldsymbol{I}_{N 1} & \mathbf{0} & \mathbf{0} & \mathbf{0} & \mathbf{0} & \mathbf{0} \\
\mathbf{0} & \mathbf{0} & \boldsymbol{\Psi}_{N_{1}} & \mathbf{0} & {\left[\boldsymbol{\Theta}_{1} \boldsymbol{\Phi}-\boldsymbol{\Psi}_{1} \boldsymbol{\Theta}_{1}\right]} & \mathbf{0} & \mathbf{0} & \mathbf{0} \\
\mathbf{0} & \mathbf{0} & \boldsymbol{I} & \mathbf{0} & \mathbf{0} & \mathbf{0} & \mathbf{0} & \mathbf{0} \\
\mathbf{0} & \mathbf{0} & \mathbf{0} & \mathbf{0} & \mathbf{\Phi} & \mathbf{0} & \mathbf{0} & \mathbf{0} \\
\mathbf{0} & \mathbf{0} & \mathbf{0} & \mathbf{0} & \boldsymbol{I} & \mathbf{0} & \mathbf{0} & \mathbf{0} \\
\mathbf{0} & \mathbf{0} & \mathbf{0} & \mathbf{0} & {\left[\boldsymbol{\Theta}_{2} \boldsymbol{\Phi}-\boldsymbol{\Psi}_{2} \boldsymbol{\Theta}_{2}\right]} & \mathbf{0} & \boldsymbol{I} & \boldsymbol{\Psi}_{2} \\
\boldsymbol{0}^{+} & \mathbf{0} & \mathbf{0} & {\left[\boldsymbol{\Theta}_{2} \boldsymbol{\Phi}-\mathbf{\Psi}_{2} \boldsymbol{\Theta}_{2}\right]} & \mathbf{0} & \mathbf{0} & \boldsymbol{\Psi}_{2}
\end{array}\right]\left[\begin{array}{c}
\boldsymbol{Y}_{1 t-1}^{c} \\
\boldsymbol{y}_{1 t-2} \\
\Delta \boldsymbol{y}_{1 t-1} \\
\Delta \boldsymbol{y}_{1 t-2} \\
\boldsymbol{f}_{t-1} \\
\boldsymbol{f}_{t-2} \\
\boldsymbol{\alpha}_{1 t-1}^{+} \\
\boldsymbol{\alpha}_{2 t-1}^{+}
\end{array}\right]} \\
& +\left[\begin{array}{c}
\boldsymbol{Y}_{1 t}^{*}-\boldsymbol{U}_{1 t}^{*} \boldsymbol{y}_{1 t}^{*}+\boldsymbol{U}_{1 t}^{*}\left(\boldsymbol{I}-\boldsymbol{\Psi}_{N_{1}}\right) \boldsymbol{\mu}_{1} \\
\mathbf{0} \\
\left(\boldsymbol{I}-\boldsymbol{\Psi}_{N_{1}}\right) \boldsymbol{\mu}_{1} \\
\mathbf{0} \\
\mathbf{0} \\
\left(\boldsymbol{I}-\mathbf{\Psi}_{2}\right) \boldsymbol{\delta}_{2} \\
\left(\boldsymbol{I}-\mathbf{\Psi}_{2}\right) \boldsymbol{\delta}_{2}
\end{array}\right]+\left[\begin{array}{ccc}
\boldsymbol{U}_{1 t} \boldsymbol{\Theta}_{1} & \boldsymbol{U}_{1 t}^{*} & \mathbf{0} \\
\mathbf{0} & \mathbf{0} & \mathbf{0} \\
\boldsymbol{\Theta}_{1} & \boldsymbol{I}_{N_{1}} & \mathbf{0} \\
\mathbf{0} & \mathbf{0} & \mathbf{0} \\
\boldsymbol{I}_{k} & \mathbf{0} & \mathbf{0} \\
\mathbf{0} & \mathbf{0} & \mathbf{0} \\
\boldsymbol{\Theta}_{2} & \mathbf{0} & \boldsymbol{I}_{N_{2}} \\
\boldsymbol{\Theta}_{2} & \mathbf{0} & \boldsymbol{I}_{N_{2}} \\
& &
\end{array}\right]\left[\begin{array}{c}
\boldsymbol{\eta}_{t} \\
\boldsymbol{\epsilon}_{1 t} \\
\boldsymbol{\epsilon}_{2 t}
\end{array}\right] .
\end{aligned}
$$

\section{Case 3. Observed at time $t$ but missing at time $t-1$}

When the observations of $\boldsymbol{y}_{2 t}$ are available but the first differences are not, due to a missing value at time $t-1$, two additional latent states are introduced, $\boldsymbol{\alpha}_{1 t}^{+{ }^{\prime}}, \boldsymbol{\alpha}_{2 t}^{+{ }^{\prime}}$. The measurement equation is modified as follows:

$$
\left[\begin{array}{c}
\boldsymbol{Y}_{1 t}^{c} \\
\Delta \boldsymbol{y}_{2 t} \\
\boldsymbol{y}_{2 t}
\end{array}\right]=\left[\begin{array}{cccccccc}
\boldsymbol{I}_{N_{1}} & \mathbf{0} & \mathbf{0} & \mathbf{0} & \mathbf{0} & \mathbf{0} & \mathbf{0} & \mathbf{0} \\
\mathbf{0} & \mathbf{0} & \mathbf{0} & \mathbf{0} & \boldsymbol{\Theta}_{2} & -\boldsymbol{\Psi}_{2} \boldsymbol{\Theta}_{2} & \mathbf{0} & \mathbf{0} \\
\mathbf{0} & \mathbf{0} & \mathbf{0} & \mathbf{0} & \mathbf{0} & \mathbf{0} & \boldsymbol{I} & \boldsymbol{\Psi}_{2}
\end{array}\right] \boldsymbol{\alpha}_{t}+\left[\begin{array}{c}
\mathbf{0} \\
\left(\boldsymbol{I}-\boldsymbol{\Psi}_{2}\right) \boldsymbol{\delta}_{2} \\
\mathbf{0}
\end{array}\right]+\left[\begin{array}{ccc}
\mathbf{0} & \mathbf{0} & \mathbf{0} \\
\mathbf{0} & \mathbf{0} & \boldsymbol{I} \\
\mathbf{0} & \mathbf{0} & \mathbf{0}
\end{array}\right]\left[\begin{array}{c}
\boldsymbol{\eta}_{t} \\
\boldsymbol{\epsilon}_{1 t} \\
\boldsymbol{\epsilon}_{2 t}
\end{array}\right]
$$

whereas the state equation becomes:

$$
\begin{aligned}
& {\left[\begin{array}{c}
\boldsymbol{Y}_{1 t}^{c} \\
\boldsymbol{y}_{1 t-1} \\
\Delta \boldsymbol{y}_{1 t} \\
\Delta \boldsymbol{y}_{1 t-1} \\
\boldsymbol{f}_{t} \\
\boldsymbol{f}_{t-1} \\
\boldsymbol{\alpha}_{1 t}^{+} \\
\boldsymbol{\alpha}_{2 t}^{+}
\end{array}\right]=\left[\begin{array}{cccccccc}
\rho_{t} \boldsymbol{I}_{N 1} & \boldsymbol{U}_{1 t}^{*} & \boldsymbol{U}_{1 t}^{*}\left(\boldsymbol{I}+\boldsymbol{\Psi}_{1}\right) & \mathbf{0} & \boldsymbol{U}_{1 t}^{*}\left[\boldsymbol{\Theta}_{1} \boldsymbol{\Phi}-\boldsymbol{\Psi}_{1} \boldsymbol{\Theta}_{1}\right] & \mathbf{0} & \mathbf{0} & \mathbf{0} \\
\mathbf{0} & \boldsymbol{I}_{N 1} & \boldsymbol{I}_{N 1} & \mathbf{0} & \mathbf{0} & \mathbf{0} & \mathbf{0} & \mathbf{0} \\
\mathbf{0} & \mathbf{0} & \boldsymbol{\Psi}_{N_{1}} & \mathbf{0} & {\left[\boldsymbol{\Theta}_{1} \boldsymbol{\Phi}-\boldsymbol{\Psi}_{1} \boldsymbol{\Theta}_{1}\right]} & \mathbf{0} & \mathbf{0} & \mathbf{0} \\
\mathbf{0} & \mathbf{0} & \boldsymbol{I} & \mathbf{0} & \mathbf{0} & \mathbf{0} & \mathbf{0} & \mathbf{0} \\
\mathbf{0} & \mathbf{0} & \mathbf{0} & \mathbf{0} & \mathbf{\Phi} & \mathbf{0} & \mathbf{0} & \mathbf{0} \\
\mathbf{0} & \mathbf{0} & \mathbf{0} & \mathbf{0} & \boldsymbol{I} & \mathbf{0} & \mathbf{0} & \mathbf{0} \\
\mathbf{0} & \mathbf{0} & \mathbf{0} & \mathbf{0} & \boldsymbol{\Theta}_{2} & -\boldsymbol{\Psi}_{2} \boldsymbol{\Theta}_{2} & \mathbf{0} & \mathbf{0} \\
\mathbf{0}^{+} & \mathbf{0} & \mathbf{0} & \mathbf{0} & \mathbf{0} & \boldsymbol{I} & \boldsymbol{\Psi}_{2}
\end{array}\right]\left[\begin{array}{c}
\boldsymbol{Y}_{1 t-1}^{c} \\
\boldsymbol{y}_{1 t-2} \\
\Delta \boldsymbol{y}_{1 t-1} \\
\Delta \boldsymbol{y}_{1 t-2} \\
\boldsymbol{f}_{t-1} \\
\boldsymbol{f}_{t-2} \\
\boldsymbol{\alpha}_{1 t-1}^{+} \\
\boldsymbol{\alpha}_{2 t-1}^{+}
\end{array}\right]} \\
& +\left[\begin{array}{c}
\boldsymbol{Y}_{1 t}^{*}-\boldsymbol{U}_{1 t}^{*} \boldsymbol{y}_{1 t}^{*}+\boldsymbol{U}_{1 t}^{*}\left(\boldsymbol{I}-\boldsymbol{\Psi}_{N_{1}}\right) \boldsymbol{\delta}_{1} \\
\mathbf{0} \\
\left(\boldsymbol{I}-\boldsymbol{\Psi}_{N_{1}}\right) \boldsymbol{\delta}_{1} \\
\mathbf{0} \\
\mathbf{0} \\
\left(\boldsymbol{I}-\boldsymbol{\Psi}_{2}\right) \boldsymbol{\delta}_{2} \\
\left(\boldsymbol{I}-\boldsymbol{\Psi}_{2}\right) \boldsymbol{\delta}_{2}
\end{array}\right]+\left[\begin{array}{ccc}
\boldsymbol{U}_{1 t} \boldsymbol{\Theta}_{1} & \boldsymbol{U}_{1 t}^{*} & \mathbf{0} \\
\mathbf{0} & \mathbf{0} & \mathbf{0} \\
\boldsymbol{\Theta}_{1} & \boldsymbol{I}_{N_{1}} & \mathbf{0} \\
\mathbf{0} & \mathbf{0} & \mathbf{0} \\
\boldsymbol{I}_{k} & \mathbf{0} & \mathbf{0} \\
\mathbf{0} & \mathbf{0} & \mathbf{0} \\
\boldsymbol{\Theta}_{2} & \mathbf{0} & \boldsymbol{I}_{N_{2}} \\
\boldsymbol{\Theta}_{2} & \mathbf{0} & \boldsymbol{I}_{N_{2}}
\end{array}\right]\left[\begin{array}{c}
\boldsymbol{\eta}_{t} \\
\boldsymbol{\epsilon}_{1 t} \\
\boldsymbol{\epsilon}_{2 t}
\end{array}\right]
\end{aligned}
$$


When there are no further missing values, the components $\boldsymbol{\alpha}_{1 t}^{+}$and $\boldsymbol{\alpha}_{2 t}^{+}$are removed and the state space formulation collapses to Eqs. (11)-(12).

\section{Appendix D: Chained GDP estimates}

We start by indexing the month of the year by $j, j=0, \ldots, 11$, and the year by $m, m=1, \ldots, M=$ $[(n+1) / 12]$, so that the time index is written $t=j+12 m, t=0, \ldots, n$.

For a particular estimated monthly time series, let us denote the value at current prices of month $j$ in year $m$ by $Y_{j m}$, the annual total by $Y_{. m}=\sum_{j} Y_{j m}$, and the annual average (the annual and quarterly figures are available from the national accounts, compiled by Eurostat) by $\bar{Y}_{m}=Y_{. m} / 12$. The chainlinked volume estimate with reference year $b$ (the year 2000 in our case) will be denoted $\hat{Y}_{j m}^{(b)}$. The temporal disaggregation methods described in the paper are applied to the quarterly chained-linked volume series with reference year $b$ and yield estimates that add up to the quarterly and annual totals (temporal consistency), but are not additive in a horizontal (that is, cross-sectional) sense.

The following multi-step procedure enables the computation of volume measures expressed at the prices of the previous year that are additive, also horizontally.

\section{Dechaining:}

(a) Transform the monthly estimates into Laspeyres-type quantity indices with reference year $b$ (volumes are evaluated at year $b$ average prices), by computing

$$
I_{j m}^{(b)}=\frac{\hat{Y}_{j m}^{(b)}}{\bar{Y}_{. b}}, \quad j=0, \ldots, 11, m=0, \ldots, M
$$

where the denominator is the annual average of year $b$ at current prices. In our case, $b=5$ (year 5 is the calendar year 2000).

(b) Change the reference year to $m=1$, the second year of the series (1996 in our case), by computing:

$$
I_{j m}^{(1)}=\frac{I_{j m}^{(b)}}{\bar{I}_{1}^{(b)}}, \quad j=1, \ldots, 11, m=0, \ldots, M,
$$

where $\bar{I}_{1}^{(b)}=\sum_{j} I_{j 1}^{(b)} / 12$ is the average quantity index for the second year of the the sample.

(c) Transform the quantity indices for year $m=1,2, \ldots, M$ into indices with reference year $m-1$ (the previous year), by rescaling $I_{j m}^{(1)}$ as follows:

$$
I_{j m}^{(m-1)}=\frac{I_{j m}^{(1)}}{\bar{I}_{m-1}^{(1)}}, \quad j=0, \ldots, 11, \quad m=1, \ldots, M,
$$

where

$$
\bar{I}_{m-1}^{(1)}=\frac{1}{12} \sum_{j} I_{j, m-1}^{(1)}, \quad m=1, \ldots, M .
$$

(d) Compute the series at the average prices of the previous year as:

$$
\hat{Y}_{j m}^{(m-1)}=I_{j m}^{(m-1)} \bar{Y}_{m-1}, \quad j=0, \ldots, 11, \quad m=1, \ldots, M .
$$


2. Aggregation step: Let $\boldsymbol{Y}_{t}^{(m-1)}$ denote the disaggregate time series expressed at the average prices of the previous year. Using the original estimates and the dechaining procedure, we can assume that, at least approximately,

$$
\boldsymbol{Y}_{t}^{(m-1)} \sim \mathrm{N}\left(\hat{\boldsymbol{Y}}_{t}^{(m-1)}, \hat{\boldsymbol{V}}_{t}^{(m-1)}\right), \quad t=0,1, \ldots, n,
$$

where the first and second moments are given by the sequential constrained estimates produced by the Kalman filter and smoother outlined in the previous section, modified to take the dechaining procedure into account. If the $r$ cross-sectional constraints are expressed as

$$
Q Y_{t}=\boldsymbol{q}
$$

where $\boldsymbol{Q}$ is an $r \times N_{1}$ matrix, and $\boldsymbol{q}$ is $r \times 1$, the modified estimates that comply with those constraints and their MSE matrix are given respectively by

$$
\begin{aligned}
& \tilde{\boldsymbol{Y}}_{t}^{(m-1)}=\hat{\boldsymbol{Y}}_{t}^{(m-1)}+\hat{\boldsymbol{V}}_{t}^{(m-1)} \boldsymbol{Q}^{\prime}\left(\boldsymbol{Q} \hat{\boldsymbol{V}}_{t}^{(m-1)} \boldsymbol{Q}^{\prime-1}\left(\boldsymbol{q}-\boldsymbol{Q} \hat{\boldsymbol{Y}}_{t}^{(m-1)}\right)\right. \\
& \tilde{\boldsymbol{V}}_{t}^{(m-1)}=\hat{\boldsymbol{V}}_{t}^{(m-1)}-\hat{\boldsymbol{V}}_{t}^{(m-1)} \boldsymbol{Q}^{\prime}\left(\boldsymbol{Q} \hat{\boldsymbol{V}}_{t}^{(m-1)} \boldsymbol{Q}^{\prime-1} \boldsymbol{Q} \hat{\boldsymbol{V}}_{t}^{(m-1)},\right.
\end{aligned}
$$

see for example ?. In our case, for each country and the Euro area, $r=2, \boldsymbol{q}=\mathbf{0}$, and

$$
\boldsymbol{Q}=\left[\begin{array}{llllllllllrr}
1 & 1 & 1 & 1 & 1 & 1 & 1 & 0 & 0 & 0 & 0 & -1 \\
0 & 0 & 0 & 0 & 0 & 0 & 0 & 1 & 1 & 1 & -1 & -1
\end{array}\right]
$$

if the GDP components are arranged as in Section 4 and the last element represents GDP at market prices. The new balanced estimates are now ready to be expressed at the average prices of reference year $b$.

3. Chain-linking (annual overlap):

(a) Convert the aggregated volume measures into Laspeyres-type quantity indices with respect to the previous year:

$$
\mathcal{I}_{j m}^{(m-1)}=\frac{\tilde{Y}_{j m}^{(m-1)}}{\bar{Y}_{m-1}}, \quad j=0, \ldots, 11, \quad m=1, \ldots, M,
$$

where $\bar{Y}_{m-1}=\sum_{j} Y_{j, m-1} / 12$ is the average of the previous year at current prices. The annual and quarterly totals are available from the national accounts compiled by Eurostat.

(b) Chain-link the indices using the recursive formula (the first year is the reference year):

$$
\mathcal{I}_{j m}^{(0)}=\mathcal{I}_{j m}^{(m-1)} \overline{\mathcal{I}}_{m-1}^{(0)}, \quad j=0, \ldots, 11, \quad m=1, \ldots, M,
$$

where $\overline{\mathcal{I}}_{0}^{(0)}=1$ and

$$
\overline{\mathcal{I}}_{m-1}^{(0)}=\frac{1}{12} \sum_{j} \mathcal{I}_{j, m-1}^{(0)} .
$$

(c) If $b>0$, then change the reference year to year $b$ :

$$
\mathcal{I}_{j m}^{(b)}=\frac{\mathcal{I}_{j m}^{(0)}}{\overline{\mathcal{I}}_{b}^{(0)}}, \quad j=0, \ldots, 11, m=1, \ldots, M .
$$


(d) Compute the chain-linked volume series with reference year $b$ :

$$
\tilde{Y}_{j m}^{(b)}=\mathcal{I}_{j m}^{(b)} \bar{Y}_{b}, \quad j=1, \ldots, 12, \quad m=2, \ldots, M,
$$

where $\bar{Y}_{b}=\frac{1}{12} \sum_{j} Y_{j b}$ is the value of GDP (at basic or market prices) at current prices of the reference year.

The multi-step procedure just described enables monthly estimates in volume to be obtained such that the values $\tilde{Y}_{j m}^{(m-1)}$ expressed at the average prices of the previous year add up to their quarterly and annual totals published by Eurostat and are consistent with the contemporaneous aggregation constraints. On the other hand, as a result of the chaining procedure, the chain-linked volumes $\tilde{Y}_{j m}^{(b)}$ expressed at the prices of the common reference year $b$ (2000) are consistent only with the temporal aggregation constraints; however, their estimates are more reliable following combination with the estimates of other related variables. 